\title{
Robustness of randomized rumour spreading ${ }^{1}$
}

\author{
Rami Daknama, Konstantinos Panagiotou* and Simon Reisser \\ Institute for Mathematics, Ludwig-Maximilians-Universität München, 80333 Munich, Germany \\ *Corresponding author. Email: kpanagio@math.lmu.de
}

(Received 27 September 2019; revised 8 June 2020; accepted 15 May 2020; first published online 12 August 2020)

\begin{abstract}
In this work we consider three well-studied broadcast protocols: push, pull and pusherpull. A key property of all these models, which is also an important reason for their popularity, is that they are presumed to be very robust, since they are simple, randomized and, crucially, do not utilize explicitly the global structure of the underlying graph. While sporadic results exist, there has been no systematic theoretical treatment quantifying the robustness of these models. Here we investigate this question with respect to two orthogonal aspects: (adversarial) modifications of the underlying graph and message transmission failures.

We explore in particular the following notion of local resilience: beginning with a graph, we investigate up to which fraction of the edges an adversary may delete at each vertex, so that the protocols need significantly more rounds to broadcast the information. Our main findings establish a separation among the three models. On one hand, pull is robust with respect to all parameters that we consider. On the other hand, push may slow down significantly, even if the adversary may modify the degrees of the vertices by an arbitrarily small positive fraction only. Finally, pushepull is robust when no message transmission failures are considered, otherwise it may be slowed down.

On the technical side, we develop two novel methods for the analysis of randomized rumour-spreading protocols. First, we exploit the notion of self-bounding functions to facilitate significantly the round-based analysis: we show that for any graph the variance of the growth of informed vertices is bounded by its expectation, so that concentration results follow immediately. Second, in order to control adversarial modifications of the graph we make use of a powerful tool from extremal graph theory, namely Szemerédi's Regularity Lemma.
\end{abstract}

2020 MSC Codes: Primary 05C85; Secondary 68R10

\section{Introduction}

Randomized broadcast protocols are highly relevant for data distribution in large networks of various kinds, including technological, social and biological networks. Among many others there are three basic models in the literature, introduced in [9], [19] and [27], namely push, pull and push\&pull (or $p p$ for short). Consider a connected graph in which some vertex holds a piece of information; we call this vertex (initially) informed. All three models have the common characteristic that they proceed in rounds. In the push model, in every round every informed vertex chooses a neighbour independently and uniformly at random and informs it; this of course only

\footnotetext{
${ }^{1}$ An extended abstract of this paper was published in the Proceedings of the European Symposium on Algorithms 2019 (ESA '19).

(C) The Author(s), 2020. Published by Cambridge University Press. This is an Open Access article, distributed under the terms of the Creative Commons Attribution licence (http://creativecommons.org/licenses/by/4.0/), which permits unrestricted re-use, distribution, and reproduction in any medium, provided the original work is properly cited.
} 
has an effect if the target vertex was previously uninformed. Conversely, in the pull model every round every uninformed vertex chooses a neighbour independently and uniformly at random and asks for the information. If the asked vertex has the information, then the asking vertex becomes informed as well. The third model, pusherpull, combines both worlds: in each round, each vertex chooses a neighbour independently and uniformly at random, and if one of two vertices is informed, then afterwards both become so. We additionally assume that each message transmission succeeds independently with probability $q \in(0,1]$. For these algorithms, the main parameter that we consider is the random variable that counts how many rounds are needed until all vertices are informed, and we call these quantities the runtimes of the respective algorithms.

In the remainder we will denote the runtime of push by $T_{\text {push }}(G, v, q)$, where $G$ is the underlying graph, initially the vertex $v$ is informed, and we have a transmission success probability of $q \in$ $(0,1]$. Analogously we denote the runtimes of pull and pushe $p u l l$ by $T_{p u l l}(G, v, q)$ and $T_{p p}(G, v, q)$ respectively. If the choice of $v$ does not matter we will omit it in our notation. The most basic case is when $G$ is the complete graph $K_{n}$ with $n$ vertices. Then (see e.g. Doerr and Kostrygin [11]) it is known that, for $\mathcal{P} \in\{p u s h, p u l l, p p\}$ and $q \in(0,1]$ in expectation and with probability tending to 1 as $n \rightarrow \infty$,

$$
T_{\mathcal{P}}\left(K_{n}, q\right)=c_{\mathcal{P}}(q) \log n+o(\log n)
$$

where, for $q \in(0,1)$,

$$
\begin{aligned}
c_{\text {push }}(q) & :=\frac{1}{\log (1+q)}+\frac{1}{q}, \\
c_{\text {pull }}(q) & :=\frac{1}{\log (1+q)}-\frac{1}{\log (1-q)}, \\
c_{p p}(q) & :=\frac{1}{\log (1+2 q)}+\frac{1}{q-\log (1-q)},
\end{aligned}
$$

and where we set $c_{\mathcal{P}}(1):=\lim _{q \rightarrow 1} c_{\mathcal{P}}(q)$. If $q$ is clear from the context, we write $c_{\mathcal{P}}$ instead of $c_{\mathcal{P}}(q)$. In fact, the results in [11] and also [12] are much more precise, but the stated forms will be sufficient for what follows.

Contribution and related work. In this article our focus is on quantifying the robustness of all three models. Indeed, robustness is a key property that is often attributed to them, since they are simple, randomized and, crucially, do not exploit explicitly the structure of the underlying graph (apart from considering the neighbourhoods of the vertices, of course). Clearly the runtime can vary tremendously between different graphs with the same number of vertices. Hence it is essential to understand the impact of structural graph characteristics on the runtime of rumour-spreading algorithms.

One result in this spirit for the push model was shown in [28]. Roughly speaking, in that paper it is shown that even on graphs with low density, if the edges are distributed rather uniformly, then push is as fast as on the complete graph. This can be interpreted as a robustness result: starting with a complete graph, one can delete a vast amount of edges, and as long as this is done rather uniformly, the runtime of push is affected insignificantly. To state the result more precisely, we need the following notion.

Definition 1.1 $((n, \delta, \Delta, \lambda)$-graph). Let $\mathrm{G}$ be a connected graph with $n$ vertices that has minimum degree $\delta$ and maximum degree $\Delta$. Let $\mu_{1} \geqslant \mu_{2} \geqslant \cdots \geqslant \mu_{n}$ be the eigenvalues of the adjacency matrix of $G$, and set

$$
\lambda=\max _{2 \leqslant i \leqslant n}\left|\mu_{i}\right|=\max \left\{\left|\mu_{2}\right|,\left|\mu_{n}\right|\right\} .
$$

We will call $G$ an $(n, \delta, \Delta, \lambda)$-graph. 
In this paper we are interested in the case where $G$ gets large, that is, when $n \rightarrow \infty$. Hence all asymptotic notation in this paper is with respect to $n$; in particular, 'with high probability', or w.h.p. for short, means with probability $1-o(1)$ when $n \rightarrow \infty$.

Definition 1.2 (expander sequence). Let $\mathcal{G}=\left(G_{n}\right)_{n \in \mathbb{N}}$ be a sequence of graphs, where $G_{n}$ is a $\left(n, \delta_{n}, \Delta_{n}, \lambda_{n}\right)$-graph for each $n \in \mathbb{N}$. We say that $\mathcal{G}$ is an expander sequence if $\Delta_{n} / \delta_{n}=1+o(1)$ and $\lambda_{n}=o\left(\Delta_{n}\right)$.

Note that if we consider any sequence $\mathcal{G}=\left(G_{n}\right)_{n \in \mathbb{N}}$ of graphs this always implicitly defines $\delta_{n}, \Delta_{n}$ and $\lambda_{n}$ as in Definition 1.2. Expander graphs have found numerous applications in computer science and mathematics; see e.g. the survey [25]. If $\mathcal{G}$ is an expander sequence, then intuitively this means that for $n$ large enough, the edges of $G_{n}$ are rather uniformly distributed. For a more formal statement see Lemma 2.7. Moreover, note that our definition of expander sequences excludes the case when $\Delta_{n}$ is bounded. This is actually a necessary condition for our robustness results to hold; see [13]. With all these definitions at hand we can state the result from [28] that quantifies the robustness of push with respect to the network topology, that is, the runtime is asymptotically the same as on the complete graph $K_{n}$.

Theorem 1.1. Let $\mathcal{G}=\left(G_{n}\right)_{n \in \mathbb{N}}$ be an expander sequence. Then w.h.p.

$$
T_{\text {push }}\left(G_{n}\right)=c_{\text {push }}(1) \log n+o(\log n) .
$$

Apart from expander sequences, results in the form of Theorem 1.1 (where the asymptotic runtimes of one or more of these algorithms are determined) were also shown for sufficiently dense Erdős-Rényi random graphs [16], random regular graphs [15] as well as hypercubes [28]. Moreover, the order of the runtime on various models that describe social networks was investigated. The Chung-Lu model was studied in [17], preferential attachment graphs were explored in [10], and geometric graphs were examined in [18]. A somewhat different approach is to derive general runtime bounds that hold for all graphs and depend only on some graph parameter, e.g. conductance $[6,20]$, vertex expansion [21] or diameter [5, 14, 23]. Furthermore, several variants of push, pull and pusherpull were studied. These include vertices being restricted to answer only one pull request per round [7], vertices being allowed to contact multiple neighbours per round $[11,28]$, vertices not calling the same neighbour twice [10] and asynchronous versions $[1,2,4,29]$. Finally, besides [11], robustness of these rumour-spreading algorithms with respect to message transmission failures was also studied by Elsässer and Sauerwald in [13]. It was shown for any graph that if a message fails with probability $1-p$, then the runtime of push increases at most by a factor of $6 / p$.

In this work our focus is on three subjects concerning the robustness of rumour spreading. Our first (and not unexpected) result extends Theorem 1.1 to the runtimes of pull and pushepull. In particular, we show that none of the three protocols slows down or speeds up on graphs with good expansion properties compared to its runtime on the complete graph. This motivates us to investigate how severely a graph with good expansion properties has to be modified to increase the respective runtimes.

In our second contribution, which is also the main result and which differs from what was treated in previous works, we propose and study a novel approach to quantifying robustness. In particular, we investigate the impact of adversarial edge deletions, where we use the well-known concept of local resilience; see e.g. $[8,31]$. To be specific, we explore up to which fraction of edges an adversary needs to be allowed to delete at each vertex to slow down the process by a significant amount of time, i.e. by $\Omega(\log n)$ rounds. Here we discover a surprising dichotomy in the following sense. On the one hand, we show that neither pull and pusherpull can be slowed down by such 
adversarial edge deletions - in essentially all but trivial cases, where the fraction is so large that the graph may become (almost) disconnected. On the other hand, we demonstrate that even a small number of edge deletions is sufficient to slow down push by $\Omega(\log n)$ rounds. In other words, we find that in contrast to pull and pushepull, the push protocol is not resilient to adversarial deletions and lacks (in this specific sense) the robustness of the other two protocols.

As our third subject, we generalize the previous results by additionally considering message transmission failures that occur independently with probability $1-q \in[0,1)$. On the positive side, we show that for arbitrary $q \in(0,1]$, all three algorithms inform almost all vertices at least as fast as in an expander sequence in spite of adversarial edge deletions. However, if we want to inform all vertices, only pull is not slowed down by adversarial edge deletions for all values of $q$; $p u s h$ can be slowed down as before, and pushepull is a mixed bag, in that for $q=1$ it cannot be slowed down whereas for $q<1$ it can. Furthermore, in general it is also possible to speed up pushepull by deleting edges, which is however not surprising as the star-graph deterministically finishes in at most two rounds.

Summarizing, this work enhances previous (robustness) results, particularly the ones concerning precise asymptotic runtimes and random transmission failures. Crucially, we introduce and study the concept of local resilience as a method to investigate robustness. However, apart from that, in this paper we develop two new general methods for the analysis of rumour-spreading algorithms.

- The most common approach in the current literature for the study of the runtime is to determine the expected number of newly informed vertices in one or more rounds and to show concentration, for example by bounding the variance. Achieving this, however, is often quite complex and makes laborious and lengthy technical arguments necessary. Here we use the theory of self-bounding functions (see Section 2), which allows us to cleanly upper-bound the variance by the expected value. The argument works for all three investigated algorithms and the bound is valid for all graphs. We are certain that this method will also facilitate future work on the analysis of rumour-spreading algorithms.

- Studying the robustness of the protocols is a challenging task, as the adversary (as described previously) has various options to modify the graph, for example by introducing a high variance in the degrees of the vertices; this turns out to be particularly problematic in the case of pushepull. Here we demonstrate that such types of irregularities can be handled universally by applying a powerful tool from a completely different area, namely extremal graph theory. In particular, we use Szemerédi's Regularity Lemma (see e.g. [30]), which allows us to partition the vertex set of a graph such that nearly all pairs of sets in the partition behave nearly like perfect regular bipartite graphs. This allows us to apply our methods on these regular pairs; eventually we obtain a linear recursion that can be solved by analysing the maximal eigenvalue of the underlying matrix.

\subsection{Results}

Our first result addresses the question about how fast rumours spread on expander graphs; in order to obtain a concise statement, the occurrence of independent message transmission failures is also considered.

Theorem 1.2. Let $\mathcal{G}=\left(G_{n}\right)_{n \in \mathbb{N}}$ be an expander sequence and let $q \in(0,1]$. Then w.h.p.

(a) $T_{\text {push }}\left(G_{n}, q\right)=c_{\text {push }}(q) \log n+o(\log (n))$,

(b) $T_{\text {pull }}\left(G_{n}, q\right)=c_{\text {pull }}(q) \log n+o(\log (n))$,

(c) $T_{p p}\left(G_{n}, q\right)=c_{p p}(q) \log n+o(\log (n))$. 
The first statement is an extension of Theorem 1.1 and its proof is a straightforward adaptation of the proof in [28]. We omit it. The contribution here is the proof of (b) and (c). Next we consider the case with edge deletions in addition to the message transmission failures.

Theorem 1.3. Let $0<\varepsilon<1 / 2, q \in(0,1]$ and $\mathcal{G}=\left(G_{n}\right)_{n \in \mathbb{N}}$ be an expander sequence. Let $\tilde{\mathcal{G}}=$ $\left(\tilde{G}_{n}\right)_{n \in \mathbb{N}}$ be such that each $\tilde{G}_{n}$ is obtained by deleting edges of $G_{n}$ such that each vertex keeps at least $a(1 / 2+\varepsilon)$ fraction of its edges. Then w.h.p.

(a) $T_{\text {pull }}\left(\tilde{G}_{n}, q\right)=c_{\text {pull }}(q) \log n+o(\log n)$,

(b) $T_{p p}\left(\tilde{G}_{n}, 1\right) \leqslant c_{p p}(1) \log n+o(\log n)$, when additionally assuming that $\delta\left(G_{n}\right) \geqslant \alpha n$ for some constant $0<\alpha \leqslant 1$.

This result demonstrates unconditionally the robustness of pull, and conditionally on $q=1$ the robustness of pushepull on dense graphs, in the case of edge deletions, that is, the runtime is asymptotically the same as in the complete graph. Moreover, we even show that pusherpull may profit from edge deletions in contrast to being slowed down; see Subsection 3.6 for an example. The proof of this result, especially the statement about pushepull, is rather involved, since the original graph may become quite irregular after the edge deletions. Here we use, among many other ingredients, the aforementioned decomposition of the graph given by Szemerédi's Regularity Lemma.

Note that Theorem 1.3 does not consider push and pushépull (when $q \neq 1$ ) at all. Indeed, our next result states that in these cases the behaviour is rather different and that the algorithms may be slowed down.

Theorem 1.4. Let $\varepsilon>0$ and $q \in(0,1]$. Then there is an expander sequence $\mathcal{G}=\left(G_{n}\right)_{n \in \mathbb{N}}$ and $a$ sequence of graphs $\tilde{\mathcal{G}}=\left(\tilde{G}_{n}\right)_{n \in \mathbb{N}}$ with the following properties. Each $\tilde{G}_{n}$ is obtained by deleting edges of $G_{n}$ such that each vertex keeps at least $a(1-\varepsilon)$ fraction of its edges. Moreover, w.h.p.

(a) $T_{\text {push }}\left(\tilde{G}_{n}, q\right) \geqslant c_{\text {push }}(q) \log n+\varepsilon /(2 q) \log n+o(\log n)$,

(b) $T_{p p}\left(\tilde{G}_{n}, q\right) \geqslant c_{p p}(q) \log n+\left(\varepsilon /(8 q)-\varepsilon q^{3} / 5\right) \log n+o(\log n)$.

Nevertheless, not all hope is lost. On the positive side, the next result states that push and pushepull are able to inform almost all vertices as fast as on the complete graph in spite of adversarial edge deletions. In this sense, we obtain an almost-robustness result for these cases.

Theorem 1.5. Let $0<\varepsilon<1 / 2, q \in(0,1]$ and $\mathcal{G}=\left(G_{n}\right)_{n \in \mathbb{N}}$ be an expander sequence. Let $\tilde{\mathcal{G}}=$ $\left(\tilde{G}_{n}\right)_{n \in \mathbb{N}}$ be such that each $\tilde{G}_{n}$ is obtained by deleting edges of $G_{n}$ such that each vertex keeps at least $a(1 / 2+\varepsilon)$ fraction of its edges. For $\mathcal{P} \in\{p u s h, p p\}$, let $\tilde{T}_{\mathcal{P}}$ denote the number of rounds needed to inform at least $n-n / \log n$ vertices. Then w.h.p.

(a) $\tilde{T}_{\text {push }}\left(\tilde{G}_{n}, q\right)=\log _{1+q}(n)+o(\log n)$,

(b) $\tilde{T}_{p p}\left(\tilde{G}_{n}, q\right) \leqslant \log _{1+2 q}(n)+o(\log n)$, when additionally assuming that $\delta\left(G_{n}\right) \geqslant \alpha n$ for some $0<\alpha \leqslant 1$.

We conjecture that there is also a version of Theorem 1.5(b) that is true for push $r$ pull on sparse graphs; to be precise we conjecture that in the setting of Theorem 1.5(b), $\tilde{T}_{p p}\left(\tilde{G}_{n}\right) \leqslant \log _{1+2 q}(n)+$ $o(\log n)$, without further restrictions on $G_{n}$, that is, pushepull cannot be slowed down, informing almost all vertices.

As a final remark, note that Theorems 1.3 and 1.5 are tight in the sense that if an adversary may delete up to half of the edges at each vertex, then there are expander graphs that become 
disconnected such that their components have linear size. On those graphs a linear fraction of the vertices will remain uninformed forever.

Outline. The rest of this paper is structured as follows. In Section 2 we collect and prove several important facts; this part of the paper also contains our technical contribution concerning the analysis through self-bounding functions. In Section 3.1 we show that pull is as fast on expanders with (or without) deleted edges as it is on the complete graph. Section 3.2 treats push\&pull on expanders without deleted edges. In the remaining subsections we focus on the cases that may be slowed down by edge deletions. In Section 3.3 we show that adversarial edge deletions cannot slow down the time until push has informed almost all vertices, by giving a coupling to the case without edge deletions. Conversely, in Section 3.4 we show that the time until push has informed all vertices can be slowed down by edge deletions, even if only a few edges are deleted. Then in Section 3.5 we show that pusherpull informs almost all vertices of dense graphs fast in spite of adversarial edge deletions. We utilize a version of Szemerédi's Regularity Lemma to get a well-behaved partition of the vertex set that is suitable for performing a round-based analysis. However, if $q<1$, adversarial edge deletions can slow down or speed up the time until push\&pull has informed all vertices for nearly all values of $q$; we show this in Section 3.6.

Further notation. Let $G=(V, E)$ denote a graph with vertex set $V$ and edge set $E \subseteq\left(\begin{array}{l}V \\ 2\end{array}\right)$. We will denote the set of neighbours of any vertex $v \in V$ by $N_{G}(v)$ or by $N(v)$, and we will denote its degree by $d_{G}(v):=\left|N_{G}(v)\right|$ or by $d(v) ; \delta_{G}$ or $\delta$ and $\Delta_{G}$ or $\Delta$ denote the minimum and maximum degree of $G$. Similarly the neighbourhood of any set of vertices $S \subseteq V$ is defined by $N_{G}(S):=\cup_{v \in S} N_{G}(v)$. Furthermore, let $U, W \subseteq V$ with $U \cap W=\emptyset$ be two disjoint vertex sets; then $E(U, W)=E_{G}(U, W)$ denotes the set of edges with one vertex in $U$ and one vertex in $W$ and let $e(U, W)=e_{G}(U, W):=\left|E_{G}(U, W)\right|$. With $E_{G}(U)$ we denote the set of edges with both vertices in $U ; e_{G}(U):=\left|E_{G}(U)\right|$. For any round $t \in \mathbb{N}$ and $\mathcal{P} \in\{p u s h, p u l l, p p\}$, we let $I_{t}^{(\mathcal{P})}(G)$ denote the set of vertices of $G$ informed by push, pull and pusherpull respectively at the beginning of round $t$ and $\left|I_{1}^{(\mathcal{P})}\right|=1$; if the underlying graph is clear from the context we will omit it; if we consider a sequence of graphs $\mathcal{G}=\left(G_{n}\right)_{n \in \mathbb{N}}$ and a sequence of times $t=(t(n))_{n \in \mathbb{N}}$, then

$$
I_{t}^{(\mathcal{P})}(\mathcal{G})=\left(I_{t(n)}^{(\mathcal{P})}\left(G_{n}\right)\right)_{n \in \mathbb{N}}
$$

is also a sequence. Similarly, $U_{t}^{(\mathcal{P})}:=V \backslash I_{t}^{(\mathcal{P})}$ denotes the set of uninformed vertices. By log we refer to the natural logarithm. For any event $A$ we will write $\mathbb{E}_{t}[A]$ instead of $\mathbb{E}\left[A \mid I_{t}\right]$ for the conditional expectation and $\mathbb{P}_{t}[A]$ instead of $\mathbb{P}\left[A \mid I_{t}\right]$ for the conditional probability. Finally we want to clarify our use of Landau symbols. Let $a, b \in \mathbb{R}$ and $f$ be a function. The terms $a \leqslant b+o(f)$ and $a \geqslant b-o(f)$ mean that there exist positive functions $g, h \in o(f)$ such that $a \leqslant b+g$ and $a \geqslant$ $b-h$. Consequently $a=b+o(f)$ means that there exists a positive function $g \in o(f)$ such that $a \in[b-g, b+g]$.

\section{Tools and techniques}

In this section we collect and prove statements about our protocols and properties of expander sequences. We begin by applying the previously mentioned notion of self-bounding functions to derive universal and simple-to-apply concentration results for our random variables, i.e. the number of informed vertices after a particular round. Then we extend the concentration results to more than one round. In the last part we recall the well-known Expander Mixing Lemma and utilize it to derive properties (weak expansion, path enumeration) for the case where we delete edges from our graphs. 
Self-bounding functions. Our main technical new result in this section is the following bound on the variance for the number of informed vertices in any given round; it is true for any graph and any set of informed vertices.

Lemma 2.1. Let $G$ be a graph, $t \in \mathbb{N}$ and $I_{t}=I_{t}^{(\mathcal{P})}(G)$ for $\mathcal{P} \in\{$ push, pull, $p p\}$. Then

$$
\operatorname{Var}\left[\left|I_{t+1}\right| \mid I_{t}\right] \leqslant \mathbb{E}\left[\left|I_{t+1}\right| \mid I_{t}\right] .
$$

Lemma 2.1 follows directly from Lemmas 2.2 and 2.3. Before stating them we introduce the notion of self-bounding functions.

Definition 2.1 (self-bounding function). Let $X$ be a set and $m \in \mathbb{N}$. A non-negative function $f: X^{m} \rightarrow \mathbb{R}$ is self-bounding if there exist functions $f_{i}: X^{m-1} \rightarrow \mathbb{R}$ such that, for all $x_{1}, \ldots, x_{m} \in X$ and all $i=1, \ldots, m$,

$$
0 \leqslant f\left(x_{1}, \ldots, x_{m}\right)-f_{i}\left(x_{1}, \ldots, x_{i-1}, x_{i+1}, \ldots, x_{m}\right) \leqslant 1
$$

and

$$
\sum_{1 \leqslant i \leqslant m}\left(f\left(x_{1}, \ldots, x_{m}\right)-f_{i}\left(x_{1}, \ldots, x_{i-1}, x_{i+1}, \ldots, x_{m}\right)\right) \leqslant f\left(x_{1}, \ldots, x_{m}\right)
$$

A striking property of self-bounding functions is the following bound on the variance.

Lemma 2.2 ([3]). For a self-bounding function $f$ and independent random variables $X_{1}, \ldots, X_{m}$, $m \in \mathbb{N}$,

$$
\operatorname{Var}\left[f\left(X_{1}, \ldots, X_{m}\right)\right] \leqslant \mathbb{E}\left[f\left(X_{1}, \ldots, X_{m}\right)\right]
$$

Lemma 2.3. Let $G$ be a graph, $t \in \mathbb{N}$, and let $I_{t}=I_{t}^{(\mathcal{P})}(G)$ for $\mathcal{P} \in\{$ push, pull, $p p\}$. Then, conditional on $I_{t}$, there exists $m \in \mathbb{N}$, independent random variables $X_{1}, \ldots, X_{m}$ and a self-bounding function $f=f^{(\mathcal{P})}$ such that $\left|I_{t+1}\right|=f\left(X_{1}, \ldots, X_{m}\right)$.

Proof. We will prove in detail the result for push, and then we show what needs to be modified in order to obtain the statement in the case of pull and pusherpull. Let $I_{t}=I_{t}^{(p u s h)}, n \in \mathbb{N}$ be the number of vertices of $G$, i.e. $V=[n]$, and $f:[n]^{\left|I_{t}\right|} \rightarrow \mathbb{R}$ with

$$
\left(x_{1}, \ldots, x_{\left|I_{t}\right|}\right) \mapsto\left|I_{t}\right|+\sum_{1 \leqslant k \leqslant\left|I_{t}\right|} \mathbb{1}\left[x_{k} \in U_{t}\right] \mathbb{1}\left[\forall \ell<k: x_{k} \neq x_{\ell}\right] .
$$

Moreover, let $\left(X_{i}\right)_{1 \leqslant i \leqslant\left|I_{t}\right|}$ be independent random variables, where $X_{i}$ is a uniformly random neighbour of the $i$ th vertex - according to an arbitrary ordering - in $I_{t}$. We argue that $f\left(X_{1}, \ldots, X_{\left|I_{t}\right|}\right)=\left|I_{t+1}\right|$. Consider $v \in I_{t}$; then $v$ is counted by the $\left|I_{t}\right|$ term in $f$. For $v \in I_{t+1} \backslash I_{t}$, let $v_{1}, \ldots, v_{s} \in I_{t}, s \in \mathbb{N}$ be the informed vertices with random neighbour $v$ in round $t$, i.e. $X_{v_{1}}=$ $\cdots=X_{v_{s}}=v$ and $X_{u} \neq v$ for all other $u \in I_{t}$. Assume further that $v_{1}<v_{2}<\cdots<v_{s}$. For $k=v_{1}$ the term $\mathbb{1}\left[X_{k} \in U_{t}\right] \mathbb{1}\left[\forall \ell<k: x_{k} \neq x_{\ell}\right]=1$ as $X_{v_{1}}=v \in U_{t}$ and for all $i \leqslant v_{1}$ it holds that $X_{i} \neq X_{v_{i}}$. For $k=v_{r}, 2 \leqslant r \leqslant s$ the term $\mathbb{1}\left[\forall \ell<k: x_{k} \neq x_{\ell}\right]=0$ as $v_{1}<v_{r}$ and $X_{v_{1}}=X_{v_{r}}=v$. Thus every vertex $v \in I_{t+1} \backslash I_{t}$ is counted exactly once by $f$. Further, set

$$
f_{i}\left(x_{1}, \ldots, x_{i-1}, x_{i+1}, \ldots, x_{\left|I_{t}\right|}\right)=\left|I_{t}\right|+\sum_{k=1, k \neq i}^{\left|I_{t}\right|} \mathbb{1}\left[x_{k} \in U_{t}\right] \mathbb{1}\left[\forall j<k, j \neq i: x_{j} \neq x_{k}\right], \quad 1 \leqslant i \leqslant\left|I_{t}\right| .
$$


The function $f_{i}$ arises from $f$ by leaving the $i$ th variable out of consideration, that is, the push of the $i$ th vertex has no effect. Then by definition $f-f_{i} \in\{0,1\}$ for all $1 \leqslant i \leqslant\left|I_{t}\right|$, and in fact we have

$$
f-f_{i}=\mathbb{1}\left[x_{i} \in U_{t}\right] \mathbb{1}\left[\forall j \neq i: x_{i} \neq x_{j}\right] .
$$

This quantity is precisely the difference in informed vertices after round $t$, assuming the $i$ th vertex did not push. Furthermore

$$
\sum_{1 \leqslant i \leqslant\left|I_{t}\right|}\left(f-f_{i}\right) \leqslant \sum_{1 \leqslant i \leqslant\left|I_{t}\right|} \mathbb{1}\left[x_{i} \in U_{t}\right] \mathbb{1}\left[\forall j \neq i: x_{i} \neq x_{j}\right] \leqslant f .
$$

Thus $f$ has the self-bounding property, which establishes the claim in the case of push. The proof for pull is completely analogous, where we use

$$
f^{(\text {pull })}:[n]^{\left|U_{t}\right|} \rightarrow \mathbb{R}, \quad\left(x_{1}, \ldots, x_{\left|U_{t}\right|}\right) \mapsto\left|I_{t}\right|+\sum_{k \in U_{t}} \mathbb{1}\left[x_{k} \in I_{t}\right]
$$

and, similarly, for pushépull we use $f^{(p p)}:[n]^{n} \rightarrow \mathbb{R}$ with

$$
\begin{aligned}
\left(x_{1}, \ldots, x_{n}\right) \mapsto & \left|I_{t}\right|+\sum_{1 \leqslant k \leqslant n} \mathbb{1}\left[k \in I_{t}\right] \mathbb{1}\left[x_{k} \in U_{t}\right] \mathbb{1}\left[\forall j \in\{1, \ldots, k\} \cap I_{t}: x_{k} \neq x_{j}\right] \\
& +\sum_{1 \leqslant k \leqslant n} \mathbb{1}\left[k \in U_{t}\right] \mathbb{1}\left[x_{k} \in I_{t}\right] \mathbb{1}\left[\forall w \in I_{t}: x_{w} \neq k\right] .
\end{aligned}
$$

Here it is useful to see that the two sums in $f^{(p p)}$ are complementary, that is, only one of the summands for index $k$ can be 1 . Thus the functions $f_{i}^{(p u l l)}$ and $f_{i}^{(p p)}$ are obtained analogously to the push case.

Remark 2.1. Let $G=(V, E)$ be a graph. Lemma 2.3 also applies to subsets of $I_{t+1}$, that is, for any $U \subset V$ and conditioned on $I_{t}$ we have that $\left|I_{t+1} \cap U\right|$ and $\left|\left(I_{t+1} \cap U\right) \backslash I_{t}\right|$ are self-bounding.

The following proposition gives a tool that we will use in order to extend our round-wise analysis to longer phases.

Proposition 2.4. Let $\left(\mathcal{A}_{i}\right)_{i \in \mathbb{N}_{0}}$ be a sequence of events, $0<c<1, \delta>0$ and $t_{1} \geqslant t_{0} \geqslant 1$, such that

$$
\mathbb{P}\left[\mathcal{A}_{t} \mid \mathcal{A}_{t_{0}}, \ldots, \mathcal{A}_{t-1}, \mathcal{A}_{0}\right] \geqslant 1-c^{t-t_{0}} \delta \text { for all } t_{0} \leqslant t \leqslant t_{1} .
$$

Then

$$
\mathbb{P}\left[\bigcap_{t=t_{0}}^{t_{1}} \mathcal{A}_{t} \mid \mathcal{A}_{0}\right] \geqslant 1-\delta /(1-c)
$$

Proof. Using the definition of conditional probability we obtain, as $c<1$,

$$
\begin{aligned}
\mathbb{P}\left[\bigcap_{t=t_{0}}^{t_{1}} \mathcal{A}_{t} \mid \mathcal{A}_{0}\right] & =\prod_{t=t_{0}}^{t_{1}} \mathbb{P}\left[\mathcal{A}_{t} \mid \mathcal{A}_{t_{0}}, \ldots, \mathcal{A}_{t-1}, \mathcal{A}_{0}\right] \\
& \geqslant \prod_{t=t_{0}}^{t_{1}}\left(1-c^{t-t_{0}} \delta\right) \\
& \geqslant 1-\sum_{t=t_{0}}^{t_{1}}\left(c^{t-t_{0}} \delta\right)
\end{aligned}
$$




$$
\begin{aligned}
& =1-\delta \sum_{t=0}^{t_{1}-t_{0}} c^{t} \\
& \geqslant 1-\delta /(1-c) .
\end{aligned}
$$

We give two typical applications of the previous lemmas, similar to what we will encounter several times later in the paper. The first lemma addresses the case where we have a lower bound for the expected number of informed vertices after one round.

Lemma 2.5. Let $\mathcal{P} \in\{$ push, pull, $p p\}$ and $I_{t}=I_{t}^{(\mathcal{P})}$. Assume that there is $c>1$ such that $\mathbb{E}_{t}\left[\left|I_{t+1}\right|\right] \geqslant$ $c\left|I_{t}\right|$ for all $t$ as long as $n / f(n) \leqslant\left|I_{t}\right| \leqslant n / g(n)$ for some functions $1 \leqslant f(n) \leqslant g(n) \leqslant n, f=o(n)$. Assuming $\left|I_{t_{0}}\right| \geqslant n / f(n)$, then there is $\tau=\log _{c}(f(n) / g(n))+o(\log n)$ such that w.h.p.

$$
\left|I_{t_{0}+\tau}\right| \geqslant n / g(n)
$$

Proof. Let $t \geqslant t_{0}$ and $n / f(n) \leqslant\left|I_{t}\right| \leqslant n / g(n)$. Lemma 2.1 guarantees that $\operatorname{Var}_{t}\left[\left|I_{t+1}\right|\right] \leqslant \mathbb{E}_{t}\left[\left|I_{t+1}\right|\right]$, and applying Chebyshev's inequality gives

$$
\mathbb{P}_{t}\left[|| I_{t+1}\left|-\mathbb{E}_{t}\left[\left|I_{t+1}\right|\right]\right| \leqslant \mathbb{E}_{t}\left[\left|I_{t+1}\right|\right]^{2 / 3}\right] \geqslant 1-\mathbb{E}_{t}\left[\left|I_{t+1}\right|\right]^{-1 / 3} \geqslant 1-\left|I_{t}\right|^{-1 / 3}
$$

Consider the events

$$
\mathcal{A}_{t}=\left|I_{t}\right| \geqslant \mathbb{E}_{t-1}\left[\left|I_{t}\right|\right]-\mathbb{E}_{t-1}\left[\left|I_{t}\right|\right]^{2 / 3} \text { or }\left|I_{t}\right| \geqslant n / g(n)
$$

The intersection of $\mathcal{A}_{t_{0}+1}, \ldots, \mathcal{A}_{t}$ implies inductively that either $\left|I_{t}\right| \geqslant n / g(n)$ or

$$
\begin{aligned}
\left|I_{t}\right| & \geqslant\left(1-\mathbb{E}_{t-1}\left[\left|I_{t}\right|\right]^{-1 / 3}\right) \mathbb{E}_{t-1}\left[\left|I_{t}\right|\right] \\
& \geqslant\left(1-\left(c\left|I_{t-1}\right|\right)^{-1 / 3}\right) c\left|I_{t-1}\right| \\
& \geqslant\left(\left(1-\left(c\left|I_{t_{0}}\right|\right)^{-1 / 3}\right) c\right)^{t-t_{0}}\left|I_{t_{0}}\right| .
\end{aligned}
$$

We obtain with (2.1)

$$
\mathbb{P}_{t_{0}}\left[\mathcal{A}_{t+1}\left|\mathcal{A}_{t_{0}+1}, \ldots, \mathcal{A}_{t},\right| I_{t} \mid<n / g(n)\right] \geqslant 1-\left(\left(1-\left(c\left|I_{t_{0}}\right|\right)^{-1 / 3}\right) c\right)^{-\left(t-t_{0}\right) / 3}\left|I_{t_{0}}\right|^{-1 / 3}
$$

and otherwise

$$
\mathbb{P}_{t_{0}}\left[\mathcal{A}_{t+1}\left|\mathcal{A}_{t_{0}+1}, \ldots, \mathcal{A}_{t},\right| I_{t} \mid \geqslant n / g(n)\right]=1
$$

Choose

$$
\tau:=t-t_{0}=\log _{c}(f(n) / g(n))+o(\log n)
$$

as small as possible such that the lower bound for $\left|I_{t+1}\right|$ in $(2.2)$ is $\geqslant n / g(n)$, that is, the lower bound in (2.2) is $<n / g(n)$ for $t=t_{0}+\tau$. Combining the two conditional probabilities we obtain for all $t_{0} \leqslant t \leqslant t_{0}+\tau$ 


$$
\mathbb{P}_{t_{0}}\left[\mathcal{A}_{t+1} \mid \mathcal{A}_{t_{0}+1}, \ldots, \mathcal{A}_{t}\right] \geqslant 1-\left(\left(1-\left(c\left|I_{t_{0}}\right|\right)^{-1 / 3}\right) c\right)^{-\left(t-t_{0}\right) / 3}\left|I_{t_{0}}\right|^{-1 / 3} .
$$

Applying Proposition 2.4 then yields the claim.

In the second lemma we make the stronger assumption that we can determine asymptotically the expected number of informed vertices after one round. Here we assume that we begin with a 'small' set of informed vertices, say of size $\sqrt{\log n}$, and want to reach a set of size nearly linear in $n$.

Lemma 2.6. Assume that there is some $c>1$ such that $\mathbb{E}_{t}\left[\left|I_{t+1}\right|\right]=(1+o(1)) c\left|I_{t}\right|$ for all $t$ as long as $\sqrt{\log n} \leqslant\left|I_{t}\right| \leqslant n / \log n$. Assume furthermore that $\left|I_{t_{0}}\right| \geqslant \sqrt{\log n}$. Then there are $\tau_{1}, \tau_{2}=$ $\log _{c}\left(n /\left|I_{t_{0}}\right|\right)+o(\log n)$ such that w.h.p.

$$
\left|I_{t_{0}+\tau_{1}}\right| \leqslant \frac{n}{\log n} \leqslant\left|I_{t_{0}+\tau_{2}}\right|
$$

Proof. Lemma 2.5, setting $f=n / \sqrt{\log n}$ and $g=\log n$ directly implies the existence of $\tau_{1}$. To find $\tau_{2}$, let $\mathcal{A}_{t}$ be the event ||$I_{t}\left|-\mathbb{E}_{t-1}\left[\left|I_{t}\right|\right]\right| \leqslant \mathbb{E}_{t-1}\left[\left|I_{t}\right|\right]^{2 / 3}$. There is $h(n) \in o(1)$ such that, for $c^{-}:=(1-h(n)) c$ and $c^{+}:=(1+h(n)) c$, we have that $\mathbb{E}_{t}\left[\left|I_{t+1}\right|\right] \leqslant c^{+}\left|I_{t}\right|$ and $\mathbb{E}_{t}\left[\left|I_{t+1}\right|\right] \geqslant c^{-}\left|I_{t}\right|$. Using this notation, the events $\mathcal{A}_{t_{0}+1}, \ldots, \mathcal{A}_{t+1}$ together imply inductively that

$$
\begin{aligned}
\left|I_{t+1}\right| & \leqslant\left(1+\mathbb{E}_{t}\left[\left|I_{t+1}\right|\right]^{-1 / 3}\right) \mathbb{E}_{t}\left[\left|I_{t+1}\right|\right] \\
& \leqslant\left(1+\left(c^{-}\left|I_{t}\right|\right)^{-1 / 3}\right) c^{+}\left|I_{t}\right| \\
& \leqslant\left(\left(1+\left(c^{-}\left|I_{t_{0}}\right|\right)^{-1 / 3}\right) c^{+}\right)^{t-t_{0}}\left|I_{t_{0}}\right|
\end{aligned}
$$

for all $t$ such that the right-hand side is bounded by $n / \log n$. Moreover, for all such $t$,

$$
\begin{aligned}
\left|I_{t+1}\right| & \geqslant\left(1-\mathbb{E}_{t}\left[\left|I_{t+1}\right|\right]^{-1 / 3}\right) \mathbb{E}_{t}\left[\left|I_{t+1}\right|\right] \\
& \geqslant\left(1-\left(c^{-}\left|I_{t}\right|\right)^{-1 / 3}\right) c^{-}\left|I_{t}\right| \\
& \geqslant\left(\left(1-\left(c^{-}\left|I_{t_{0}}\right|\right)^{-1 / 3}\right) c^{-}\right)^{t-t_{0}}\left|I_{t_{0}}\right| .
\end{aligned}
$$

Thus, as $\mathcal{A}_{t}$ only depends on $I_{t}$, it follows with (2.1) that

$$
\mathbb{P}_{t_{0}}\left[\mathcal{A}_{t+1} \mid \mathcal{A}_{t_{0}+1}, \ldots, \mathcal{A}_{t}\right] \geqslant 1-\left(\left(1-\left(c^{-}\left|I_{t_{0}}\right|\right)^{-1 / 3}\right) c^{-}\right)^{-\left(t-t_{0}\right) / 3}\left|I_{t_{0}}\right|^{-1 / 3} .
$$

Applying Proposition 2.4 yields the existence of $\tau_{2}$.

Expander sequences. In this section we collect some important properties of expander sequences that we are going to use later. We start by stating a version of the well-known Expander Mixing Lemma applied to our setting of expander sequences.

Lemma 2.7 ([28, Corollary 2.4]). Let $\mathcal{G}=\left(G_{n}\right)_{n \in \mathbb{N}}=\left(\left(V_{n}, E_{n}\right)\right)_{n \in \mathbb{N}}$ be an expander sequence. Then, for $S_{n} \subseteq V_{n}$ such that $1 \leqslant\left|S_{n}\right| \leqslant n / 2$, it is

$$
\left|e\left(S_{n}, V_{n} \backslash S_{n}\right)-\frac{\Delta_{n}\left|S_{n}\right|\left(n-\left|S_{n}\right|\right)}{n}\right|=o\left(\Delta_{n}\right)\left|S_{n}\right| .
$$

The following result is a consequence of the Expander Mixing Lemma that applies to graphs in which some edges were removed. It seems very simple but it turns out to be surprisingly useful.

Lemma 2.8. Let $\mathcal{G}=\left(G_{n}\right)_{n \in \mathbb{N}}=\left(\left(V_{n}, E_{n}\right)\right)_{n \in \mathbb{N}}$ be an expander sequence. Let $\varepsilon>0$ and set $\tilde{\mathcal{G}}=$ $\left(\tilde{G}_{n}\right)_{n \in \mathbb{N}}$, where each $\tilde{G}_{n}$ is obtained from $G_{n}$ by deleting edges such that each vertex keeps at least 
$a(1 / 2+\varepsilon)$ fraction of its edges. For each $n \in \mathbb{N}$ let $S_{n} \subseteq V_{n}$. Then there is $n_{0} \in \mathbb{N}$ such that, for all $n \geqslant n_{0}$,

$$
e_{\tilde{G}_{n}}\left(S_{n}, V_{n} \backslash S_{n}\right) \geqslant \varepsilon e_{G_{n}}\left(S_{n}, V_{n} \backslash S_{n}\right) .
$$

Proof. Without loss of generality we assume that $\left|S_{n}\right| \leqslant n / 2$. Since at most $(1 / 2-\varepsilon) \Delta_{n}$ edges are deleted at each vertex, we immediately obtain that

$$
e_{\tilde{G}_{n}}\left(S_{n}, V_{n} \backslash S_{n}\right) \geqslant e_{G_{n}}\left(S_{n}, V_{n} \backslash S_{n}\right)-\Delta_{n}(1 / 2-\varepsilon)\left|S_{n}\right| .
$$

Using Lemma 2.7 and choosing $n_{0}$ large enough such that

$$
\frac{o\left(\Delta_{n}\right)}{\Delta_{n}} \frac{n}{n-\left|S_{n}\right|}<\varepsilon \quad \text { for all } n \geqslant n_{0}
$$

we obtain that

$$
\begin{aligned}
& (1-\varepsilon) e_{G_{n}}\left(S_{n}, V_{N} \backslash S_{n}\right)-\Delta_{n}(1 / 2-\varepsilon)\left|S_{n}\right| \\
& \geqslant(1-\varepsilon) \frac{\Delta_{n}\left|S_{n}\right|\left(n-\left|S_{n}\right|\right)}{n}-o\left(\Delta_{n}\right)\left|S_{n}\right|-\Delta_{n}(1 / 2-\varepsilon)\left|S_{n}\right| \\
& \quad=\frac{\Delta_{n}\left|S_{n}\right|\left(n-\left|S_{n}\right|\right)}{n}\left(1-\varepsilon-\frac{o\left(\Delta_{n}\right)}{\Delta_{n}} \frac{n}{n-\left|S_{n}\right|}-\frac{n(1 / 2-\varepsilon)}{n-\left|S_{n}\right|}\right) .
\end{aligned}
$$

As $n-\left|S_{n}\right| \geqslant n / 2$, the last expression is $>0$. Hence

$$
\begin{aligned}
e_{\tilde{G}_{n}}\left(S_{n}, V_{n} \backslash S_{n}\right) & \geqslant \varepsilon e_{G}\left(S_{n}, V_{n} \backslash S_{n}\right)+(1-\varepsilon) e_{G}\left(S_{n}, V_{n} \backslash S_{n}\right)-\Delta_{n}(1 / 2-\varepsilon)\left|S_{n}\right| \\
& \geqslant \varepsilon e_{G_{n}}\left(S_{n}, V_{n} \backslash S_{n}\right) .
\end{aligned}
$$

Next we give a lemma that counts the number of paths between two arbitrary vertices of a dense graph satisfying a weak expander property (as for example guaranteed by Lemma 2.8). This will later be used to give a lower bound on the probability of any vertex being informed after a given constant number of rounds.

Lemma 2.9. Let $G=(V, E),|V|=n$. Assume that there is $\alpha>0$ such that $d(v) \geqslant \alpha n$ for all $v \in V$ and $e(W, V \backslash W) \geqslant \alpha|W||V \backslash W|$ for all $W \subseteq V$. Then, for all $u, w \in V$, there is $1 \leqslant d \leqslant 8 / \alpha^{2}+2$ such that there are at least $\left(\alpha^{4} / 64\right)^{d+1} n^{d-1}$ paths of length $d$ from $u$ to $w$.

Proof. Assume $\alpha \leqslant 1 / 2$, as otherwise the claim is trivial (with $d \in\{1,2\}$ ). We define sequences $\left(S_{i}\right)_{i \in \mathbb{N}}$ and $\left(H_{i}\right)_{i \in \mathbb{N}} \subseteq V$ as follows. Set $S_{1}=\{u\} \cup N(u), W=\{w\} \cup N(w)$ and $H_{1}=V \backslash\left(S_{1} \cup W\right)$ and proceed for $i \geqslant 1$ as follows. Let $\tilde{S}_{i+1} \subseteq H_{i}$ be the set of vertices $v \in H_{i}$ with $\left|N(v) \cap S_{i}\right| \geqslant$ $\alpha^{2} n / 8$. Set $S_{i+1}=S_{i} \cup \tilde{S}_{i+1}$ and $H_{i+1}=H_{i} \backslash \tilde{S}_{i+1}$. Then we claim that, for all $i \geqslant 1$,

$$
e\left(S_{i}, W\right) \geqslant \alpha^{3} n^{2} / 2 \text { or }\left|S_{i+1}\right| \geqslant\left|S_{i}\right|+\alpha^{2} n / 8 .
$$

To see this, assume that $e\left(S_{i}, W\right) \leqslant \alpha^{3} n^{2} / 2$. Since $\left|S_{i}\right|,|W| \geqslant \alpha n$, the weak expansion property guarantees that

$$
e\left(S_{i}, H_{i}\right)=e\left(S_{i}, H_{i} \cup W\right)-e\left(S_{i}, W\right) \geqslant \alpha\left|S_{i}\right|\left|H_{i} \cup W\right|-\alpha^{3} n^{2} / 2 \geqslant \alpha^{2}(1-\alpha) n^{2}-\alpha^{3} n^{2} / 2,
$$

and using $\alpha \leqslant 1 / 2$ we obtain that $e\left(S_{i}, H_{i}\right) \geqslant \alpha^{2} n^{2} / 4$. To complete the proof of (2.3) we compute the size of $\tilde{S}_{i+1}$. As $\left|N(v) \cap S_{i}\right| \leqslant \alpha^{2} n / 8$ for all $v \in H_{i} \backslash \tilde{S}_{i+1}$ and $\left|N(v) \cap S_{i}\right| \leqslant n$, we get

$$
\frac{\alpha^{2} n^{2}}{4} \leqslant e\left(S_{i}, H_{i}\right) \leqslant\left|\tilde{S}_{i+1}\right| n+\left|H_{i}\right| \frac{\alpha^{2} n}{8} .
$$


Since $\left|H_{i}\right| \leqslant n$ we immediately get that $\left|\tilde{S}_{i+1}\right| \geqslant \alpha^{2} n / 8$, which shows (2.3). We next show that there are (sufficiently) many paths for each vertex in $S_{i}$ to $u$. More precisely, let $1 \leqslant j \leqslant 8 / \alpha^{2}$ be such that $e\left(S_{i}, W\right)<\alpha^{3} n^{2} / 2$ for all $1 \leqslant i \leqslant j$. For those $i$ we have by $(2.3)$ that $\left|S_{i}\right| \geqslant i \cdot \alpha^{2} n / 8$. We claim that for all $v \in S_{i} \backslash\{u\}$ there is $d \leqslant i$ such that $v$ has at least $\left(\alpha^{4} / 64\right)^{d} \cdot n^{d-1}$ paths of length $d$ with endpoint $u$. We show the claim by induction on $i$. The base case $v \in S_{1} \backslash\{u\}$ is clear, as $1 \geqslant \alpha^{4} / 64$. For the induction step assume that $v \in S_{i+1} \backslash S_{i}, v \neq u$. Then by construction $\left|N(v) \cap S_{i}\right| \geqslant \alpha^{2} n / 8$. Thus, by induction hypothesis, there is $d \leqslant i$ such that $v$ has at least $\alpha^{2} n /(8 i)$ neighbours with at least $\left(\alpha^{4} / 64\right)^{d} n^{d-1}$ paths with endpoint $u$. As $i \leqslant 8 / \alpha^{2}$ this gives that $v$ has at least $\alpha^{2} n /(8 i) \cdot\left(\alpha^{4} / 64\right)^{d} n^{d-1} \geqslant\left(\alpha^{4} / 64\right)^{d+1} n^{d}$ paths of length $d+1 \leqslant i+1$ with endpoint $u$, and this accomplishes the induction step. With all these facts at hand we finally show the claim of the lemma. Let $j \leqslant 8 / \alpha^{2}$ be the first index such that $e\left(S_{j}, W\right) \geqslant \alpha^{3} n^{2} / 2$, and let $W^{\prime} \subseteq W$ be such that $\left|N(v) \cap S_{j}\right| \geqslant \alpha^{3} n / 4$ for all $v \in W^{\prime}$. Thus

$$
\frac{\alpha^{3} n^{2}}{2} \leqslant e\left(S_{j}, W\right) \leqslant\left|W^{\prime}\right| n+|W| \frac{\alpha^{3} n}{4},
$$

and thus $\left|W^{\prime}\right| \geqslant \alpha^{3} n / 4$. Then there is $d \leqslant j$ and $W^{\prime \prime} \subseteq W^{\prime}$ such that $\left|W^{\prime \prime}\right| \geqslant\left|W^{\prime}\right| / j$ and every $v$ in $W^{\prime \prime}$ has at least $\alpha^{3} n /(4 j)$ neighbours with at least $\left(\alpha^{4} / 64\right)^{d} n^{d-1}$ paths of length $d$ with endpoint $u$. Therefore every $v \in W^{\prime \prime}$ has at least

$$
\left(\alpha^{4} / 64\right)^{d} n^{d-1} \cdot \alpha^{3} n /(4 j) \geqslant\left(\alpha^{4} / 64\right)^{d+1} n^{d} / j
$$

paths of length $d+1$ with endpoint $u$. This in turn gives that there are at least

$$
\left|W^{\prime}\right| / j \cdot\left(\alpha^{4} / 64\right)^{d+1} n^{d} / j \geqslant \alpha^{3} / 4 \cdot\left(\alpha^{4} / 64\right)^{d+2} n^{d+1}
$$

paths of length $d+2$ from $w$ to $u$, and the proof is completed.

Next comes a technical lemma that, given a small set, quantifies the number of vertices for which only a small fraction of their neighbourhood intersects that given set.

Lemma 2.10. Let $\mathcal{G}=\left(G_{n}\right)_{n \in \mathbb{N}}=\left(\left(V_{n}, E_{n}\right)\right)_{n \in \mathbb{N}}$ be an expander sequence. Let $\varepsilon>0$ and let $\tilde{\mathcal{G}}=$ $\left(\tilde{G}_{n}\right)_{n \in \mathbb{N}}$, where each $\tilde{G}_{n}$ it is obtained from $G_{n}$ by deleting edges such that each vertex keeps at least $a(1 / 2+\varepsilon)$ fraction of its edges. Let $A_{n} \subseteq V_{n}$ with $\left|A_{n}\right|=o(n)$.

(a) There is $B_{n} \subseteq A_{n}$ with $\left|B_{n}\right|=(1-o(1))\left|A_{n}\right|$ such that, for all $u \in B_{n}$,

$$
\frac{\left|N_{\tilde{G}_{n}}(u) \cap A_{n}\right|}{\left|N_{\tilde{G}_{n}}(u)\right|}=o(1) .
$$

(b) There is $B_{n} \subseteq V_{n} \backslash A_{n}$ with $\left|V_{n} \backslash\left(A_{n} \cup B_{n}\right)\right|=o\left(\left|A_{n}\right|\right)$ such that, for all $v \in B_{n}$,

$$
\frac{\left|N_{\tilde{G}_{n}}(v) \cap A_{n}\right|}{\left|N_{\tilde{G}_{n}}(v)\right|}=o(1) .
$$

Proof. Let $\delta_{n}, \Delta_{n}$ denote the minimum and maximum degree of $G_{n}$. Lemma 2.7 yields that

$$
e_{G_{n}}\left(A_{n}, V_{n} \backslash A_{n}\right)=\frac{\Delta_{n}\left|A_{n}\right|\left|V_{n} \backslash A_{n}\right|}{n}+o\left(\Delta_{n}\right)\left|A_{n}\right|=(1+o(1)) \Delta_{n}\left|A_{n}\right| .
$$


As there are a maximum of $\Delta_{n}\left|A_{n}\right|$ edges with at least one point in $A_{n}$, we get that $e_{G_{n}}\left(A_{n}\right)=$ $o\left(\Delta_{n}\right)\left|A_{n}\right|$. Since we obtain $\tilde{G}_{n}$ from $G_{n}$ by deleting edges,

$$
e_{\tilde{G}_{n}}\left(A_{n}\right)=o\left(\Delta_{n}\right)\left|A_{n}\right| \text {. }
$$

With this fact at hand we show (a). Let $\eta>0$ and call a vertex $u \in A_{n}$ bad if $\left|N_{\tilde{G}_{n}}(u) \cap A_{n}\right| \geqslant$ $\eta\left|N_{\tilde{G}_{n}}(u)\right|$. Since $N_{\tilde{G}_{n}}(u) \geqslant \delta_{n} / 2$, we obtain for any bad $u$ that $\left|N_{\tilde{G}_{n}}(u) \cap A_{n}\right| \geqslant \eta \delta_{n} / 2$. As $\delta_{n}=$ $(1-o(1)) \Delta_{n}$, we infer from (2.4) that the number of bad vertices is $o\left(\left|A_{n}\right|\right)$.

To see (b), again let $\eta>0$ and this time call a vertex $v \in V_{n} \backslash A_{n}$ bad if $\left|N_{\tilde{G}_{n}}(v) \cap A_{n}\right| \geqslant$ $\eta\left|N_{\tilde{G}_{n}}(v)\right|$. Then, for any such bad $v$, we know that $\left|N_{\tilde{G}_{n}}(v) \cap A_{n}\right| \geqslant \eta \delta_{n} / 2$. As before, using (2.4) we readily get that the number of bad $v$ is $o\left(\left|A_{n}\right|\right)$.

We conclude our preliminary section by giving a lemma that crudely bounds the time needed until at least $\omega(1)$ vertices are informed.

Lemma 2.11. Let $0<\varepsilon \leqslant 1 / 2, q \in(0,1]$ and $\mathcal{G}=\left(G_{n}\right)_{n \in \mathbb{N}}$ be an expander sequence. Let $\tilde{\mathcal{G}}=$ $\left(\tilde{G}_{n}\right)_{n \in \mathbb{N}}$ be such that each $\tilde{G}_{n}$ is obtained by deleting edges of $G_{n}$ such that each vertex keeps at least a $(1 / 2+\varepsilon)$ fraction of its edges. Let $\mathcal{P} \in\{$ push, pull, pp $\}$ and suppose that $\left|I_{t}^{(\mathcal{P})}\right|<\sqrt{\log n}$. Then there is $\tau=o(\log n)$ such that w.h.p. $\left|I_{t+\tau}^{(\mathcal{P})}\right| \geqslant \sqrt{\log n}$.

Proof. Recall that the probability that $v \in U_{t}$ gets informed by pull is $q\left|N(v) \cap I_{t}\right| /|N(v)|$. Thus

$$
\mathbb{P}_{t}\left[\left|I_{t+1}^{(p u l l)} \backslash I_{t}\right|=0\right]=\prod_{u \in N\left(I_{t}\right) \cap U_{t}}\left(1-\frac{\left.q \mid N(u) \cap I_{t}\right) \mid}{|N(u)|}\right) \leqslant e^{-q e\left(U_{t}, I_{t}\right) / \Delta_{n}} .
$$

Similarly we obtain for push

$$
\mathbb{P}_{t}\left[\left|I_{t+1}^{(\text {push })} \backslash I_{t}\right|=0\right]=\prod_{v \in I_{t}} \frac{\left|N(v) \cap I_{t}\right|}{|N(v)|}=\prod_{v \in I_{t}}\left(1-\frac{\left|N(v) \cap U_{t}\right|}{|N(v)|}\right) \leqslant e^{-q e\left(I_{t}, U_{t}\right) / \Delta_{n}} .
$$

The same bound is obviously also true for pusherpull. Thus, for all $\mathcal{P} \in\{p u s h, p u l l, p p\}$,

$$
\mathbb{P}_{t}\left[\left|I_{t+1}^{(\mathcal{P})} \backslash I_{t}\right| \geqslant 1\right] \geqslant 1-e^{-q e\left(U_{t}, I_{t}\right) / \Delta_{n}} .
$$

As Lemmas 2.7 and 2.8 imply that $e\left(U_{t}, I_{t}\right) \geqslant(1+o(1)) \varepsilon \Delta_{n}\left|I_{t}\right|$, there is $c \in(0,1)$ such that $\mathbb{P}\left[\left|I_{t+1}^{(\mathcal{P})} \backslash I_{t}\right| \geqslant 1\right]>c$. Define $\tau:=\lceil(2 / c) \sqrt{\log n}\rceil$ and $X=\operatorname{Bin}(\tau, c)$ with $\mathbb{E}[X]=c \tau$ and $\operatorname{Var}[X]=$ $\tau(1-c) c$. Then, using Chebyshev,

$\mathbb{P}_{t}\left[\left|I_{t+\tau}^{(\mathcal{P})}\right| \leqslant \sqrt{\log n}\right] \leqslant \mathbb{P}_{t}[X \leqslant \sqrt{\log n}] \leqslant \mathbb{P}_{t}[|X-\mathbb{E}[X]| \leqslant \mathbb{E}[X] / 2] \leqslant 4 \operatorname{Var}[X] / \mathbb{E}[X]^{2}=o(1)$.

\section{Proofs}

\subsection{Proof of Theorems 1.2(b) and 1.3(a) - edge deletions do not slow down pull}

Let $0<\varepsilon \leqslant 1 / 2$. In this section we study the runtime of pull in the case in which the input graph is an expander, and where at each vertex at most a $(1 / 2-\varepsilon)$ fraction of the edges is deleted. The runtime on expander sequences without edge deletions, i.e. the setting in Theorem 1.2(b), is included as the special case where we set $\varepsilon=1 / 2$. In contrast to previous proofs, in the analysis of pull the 'standard' approach that consists of showing, for example, that $\mathbb{E}_{t}\left[\left|I_{t+1} \backslash I_{t}\right|\right] \approx\left|I_{t}\right|$ fails. The main reason is that the graph between $I_{t}$ and $U_{t}$ might be quite irregular, so that, depending on the actual state, $\mathbb{E}_{t}\left[\left|I_{t+1} \backslash I_{t}\right|\right] \approx c\left|I_{t}\right|$ for some $c<1$. However, we discover a different invariant that is preserved, namely that the number of edges between $I_{t}$ and $U_{t}$ behaves in an exponential way. With Lemmas 2.7 and 2.8 we can then relate this to the number of informed vertices. 
Lemma 3.1. Consider the setting of Theorem 1.3(a) and let $I_{t}=I_{t}^{(p u l l)}$.

(a) Let $\sqrt{\log n} \leqslant\left|I_{t}\right| \leqslant n / \log n$. Then

$$
\left|e\left(U_{t+1}, I_{t+1}\right)-(1+q) e\left(U_{t}, I_{t}\right)\right| \leqslant\left|I_{t}\right|^{-1 / 3} e\left(U_{t}, I_{t}\right)
$$

with probability at least $1-O\left(\left|I_{t}\right|^{-1 / 3}\right)$.

(b) Let $\left|U_{t}\right| \leqslant n / \log n$. Then $\mathbb{E}_{t}\left[\left|U_{t+1}\right|\right]=(1-q+o(1))\left|U_{t}\right|$.

Proof. We start with (a). Let $D_{t}=e\left(U_{t+1}, I_{t+1}\right)-e\left(U_{t}, I_{t}\right)$ and for $u \in U_{t}$ let $X_{u}$ be the random variable that indicates whether $u$ gets informed in round $t+1$. Then

$$
\begin{aligned}
\mathbb{E}_{t}\left[D_{t}\right] & =\sum_{u \in U_{t}} \sum_{v \in N(u) \cap U_{t}} \mathbb{E}_{t}\left[X_{u}\left(1-X_{v}\right)\right]-\sum_{u \in U_{t}} \mathbb{E}_{t}\left[X_{u}\right] \cdot\left|N(u) \cap I_{t}\right| \\
& \left.=\sum_{u \in U_{t}} q \frac{\left|N(u) \cap I_{t}\right|}{|N(u)|}\left(\sum_{v \in N(u) \cap U_{t}} 1-q \frac{\left|N(v) \cap I_{t}\right|}{|N(v)|}\right)-\left|N(u) \cap I_{t}\right|\right) .
\end{aligned}
$$

The second sum is at most $|N(u)|$, so obviously $\mathbb{E}_{t}\left[D_{t}\right] \leqslant q e\left(U_{t}, I_{t}\right)$. To get a lower bound consider a largest set $\tilde{U} \subseteq U_{t}$ such that $\left|N(u) \cap I_{t}\right| /|N(u)|=o(1)$ for all $u \in \tilde{U}$. From Lemma 2.10(b) we obtain that $\left|U_{t} \backslash \tilde{U}\right|=o\left(\left|I_{t}\right|\right)$, and so

$$
\mathbb{E}_{t}\left[D_{t}\right] \geqslant \sum_{u \in U_{t}} q\left|N(u) \cap I_{t}\right|\left(\left(\sum_{v \in N(u) \cap \tilde{U}} \frac{1}{|N(u)|}-o\left(\frac{1}{|N(u)|}\right)\right)-\frac{\left|N(u) \cap I_{t}\right|}{|N(u)|}\right) .
$$

Consider furthermore $\hat{U} \subseteq \tilde{U}$ such that $|N(u) \cap \tilde{U}| /|N(u)|=1-o(1)$ and thus also $\mid N(u) \cap$ $I_{t}|/| N(u) \mid=o(1)$ for all $u \in \hat{U}$. Lemma 2.10(b) again yields that we can choose $\hat{U}$ such that $\left|U_{t} \backslash \hat{U}\right|=o\left(\left|I_{t}\right|\right)$, and thus

$$
\begin{aligned}
\mathbb{E}_{t}\left[D_{t}\right] & \geqslant(1-o(1)) \sum_{u \in \hat{U}} q\left|N(u) \cap I_{t}\right|\left(\frac{|N(u) \cap \tilde{U}|}{|N(u)|}-\frac{\left|N(u) \cap I_{t}\right|}{|N(u)|}\right)-\sum_{u \in U_{t} \backslash \hat{U}}\left|N(u) \cap I_{t}\right| \\
& \geqslant(q-o(1)) e\left(U_{t}, I_{t}\right)-2 e\left(U_{t} \backslash \hat{U}, I_{t}\right) .
\end{aligned}
$$

According to Lemmas 2.7 and 2.8 we have that $e\left(U_{t}, I_{t}\right)=\Theta\left(\left|I_{t}\right| \Delta_{n}\right)$. But

$$
e\left(U_{t} \backslash \tilde{U}, I_{t}\right) \leqslant\left|U_{t} \backslash \tilde{U}\right| \Delta_{n}=o\left(\left|I_{t}\right| \Delta_{n}\right) .
$$

Thus $\mathbb{E}_{t}\left[e\left(U_{t+1}, I_{t+1}\right)\right]=(1+q-o(1)) e\left(U_{t}, I_{t}\right)$. In the next step we bound the variance. For each edge $e$ let $X_{e}$ be the indicator random variable that denotes the events that $e \in E\left(U_{t+1}, I_{t+1}\right)$. Thus

$$
e\left(U_{t+1}, I_{t+1}\right)=\sum_{e \in E} X_{e}=\frac{1}{2} \sum_{u \in V} \sum_{v \in N(u)} X_{\{u, v\}} .
$$

Using the fact that $X_{e}$ and $X_{e^{\prime}}$ are independent for all $e, e^{\prime} \in E$ with $e \cap e^{\prime}=\emptyset$,

$$
\begin{aligned}
\operatorname{Var}\left[e\left(U_{t+1}, I_{t+1}\right)\right] & =\operatorname{Var}\left[\sum_{e \in E} X_{e}\right] \\
& =\sum_{e, e^{\prime} \in E} \mathbb{E}\left[X_{e} X_{e^{\prime}}\right]-\mathbb{E}\left[X_{e}\right] \mathbb{E}\left[X_{e^{\prime}}\right] \\
& \leqslant \sum_{u \in V} \sum_{v, v^{\prime} \in N(u)} \mathbb{E}\left[X_{\{u, v\}} X_{\left\{u, v^{\prime}\right\}}\right]
\end{aligned}
$$




$$
\begin{aligned}
& \leqslant \Delta_{n} \sum_{u \in V} \sum_{v \in N(u)} \mathbb{E}\left[X_{\{u, v\}}\right] \\
& =2 \Delta_{n} \mathbb{E}\left[e\left(U_{t+1}, I_{t+1}\right)\right] .
\end{aligned}
$$

Since

$$
\mathbb{E}_{t}\left[e\left(U_{t+1}, I_{t+1}\right)\right]=(1+q-o(1)) e\left(U_{t}, I_{t}\right)=\Theta\left(\Delta_{n}\left|I_{t}\right|\right),
$$

by Lemmas 2.7 and 2.8 and

$$
\operatorname{Var}\left[e\left(U_{t+1}, I_{t+1}\right)\right] \leqslant 2 \Delta_{n} \mathbb{E}_{t}\left[e\left(U_{t+1}, I_{t+1}\right)\right]
$$

we immediately obtain for $\left|I_{t}\right| \geqslant \sqrt{\log n}$ with Chebyshev's inequality that

$$
\mathbb{P}\left[\left|e\left(U_{t+1}, I_{t+1}\right)-\mathbb{E}_{t}\left[e\left(U_{t+1}, I_{t+1}\right)\right]\right| \geqslant e\left(U_{t}, I_{t}\right)\left|I_{t}\right|^{-1 / 3}\right] \leqslant O\left(\left|I_{t}\right|^{-1 / 3}\right) .
$$

Next we show (b). We bound the expected number of uninformed vertices after one additional round. Lemma 2.10(a) asserts that there is a set $\tilde{U} \subseteq U_{t}$ such that $|\tilde{U}|=(1-o(1))\left|U_{t}\right|$ and $\mid N(u) \cap$ $I_{t}|/| N(u) \mid=1-o(1)$ for all $u \in \tilde{U}$. Thus

$$
\begin{aligned}
\mathbb{E}_{t}\left[\left|U_{t+1}\right|\right] & =\sum_{u \in U_{t}} 1-q \frac{\left|N(u) \cap I_{t}\right|}{|N(u)|} \\
& \leqslant\left|U_{t}\right|-q \sum_{u \in \tilde{U}} \frac{\left|N(u) \cap I_{t}\right|}{|N(u)|} \\
& =\left|U_{t}\right|-q(1-o(1))|\tilde{U}| \\
& =(1-q-o(1))\left|U_{t}\right| .
\end{aligned}
$$

As $\left|N(u) \cap I_{t}\right| \leqslant|N(u)|$ we also have

$$
\mathbb{E}_{t}\left[\left|U_{t+1}\right|\right]=\sum_{u \in U_{t}} 1-q \frac{\left|N(u) \cap I_{t}\right|}{|N(u)|} \geqslant \sum_{u \in U_{t}}(1-q)=(1-q)\left|U_{t}\right| .
$$

Lemmas 3.2 and 2.11 give lower bounds which, together with an upper bound provided by Lemma 3.3, imply Theorems 1.2(b) and 1.3(a).

Lemma 3.2. (upper bound in Theorem 1.3(a)). Consider the setting of Theorem 1.3(a) and let $I_{t}=I_{t}^{(p u l l)}$. Then the following statements hold w.h.p.

(a) Let $\sqrt{\log n} \leqslant\left|I_{t}\right| \leqslant n / \log n$. Then there are $\tau_{1}, \tau_{2}=\log _{1+q}\left(n /\left|I_{t}\right|\right)+o(\log n)$ such that $\left|I_{t+\tau_{2}}\right|<n / \log n<\left|I_{t+\tau_{1}}\right|$.

(b) Let $n / \log n \leqslant\left|I_{t}\right| \leqslant n-n / \log n$. Then there is $\tau=o(\log n)$ such that $\left|I_{t+\tau}\right|>n-n / \log n$.

(c) Let $\left|I_{t}\right| \geqslant n-n / \log n$.

(i) Case $q=1$. Then there is $\tau=o(\log n)$ such that $\left|I_{t+\tau}\right|=n$.

(ii) Case $q \neq 1$. Then there is $\tau \leqslant-\log n / \log (1-q)+o(\log n)$ such that $\left|I_{t+\tau}\right|=n$.

Proof. We start with (a). Let $\left|I_{t}\right| \in[\sqrt{\log n}, n / \log n]$. First note that any bound on $e\left(U_{t}, I_{t}\right)$ translates to a bound for $\left|I_{t}\right|$, as with Lemmas 2.7 and 2.8 we obtain

$$
(1-o(1)) \varepsilon \Delta_{n}\left|I_{t}\right| \leqslant e\left(U_{t}, I_{t}\right) \leqslant \Delta_{n}\left|I_{t}\right| \text {. }
$$

In particular, up to constant factors, $\left|I_{t}\right|$ is $e\left(U_{t}, I_{t}\right) / \Delta_{n}$ and vice versa. From Lemma 3.1(a) we obtain that $e\left(U_{t+1}, I_{t+1}\right)=\left(1+q \pm\left|I_{t}\right|^{-1 / 3}\right) e\left(U_{t}, I_{t}\right)$ with probability $1-O\left(\left|I_{t}\right|^{-1 / 3}\right)$. Proceeding as in Lemmas 2.5 and 2.6 and their proofs, where we replace the events

$$
\left|I_{t}\right| \geqslant \mathbb{E}_{t-1}\left[\left|I_{t}\right|\right]-\mathbb{E}_{t-1}\left[\left|I_{t}\right|\right]^{2 / 3} \text { or }\left|I_{t}\right| \geqslant n / g(n)
$$


and

$$
|| I_{t}\left|-\mathbb{E}_{t-1}\left[\left|I_{t}\right|\right]\right| \leqslant \mathbb{E}_{t-1}\left[\left|I_{t}\right|\right]^{2 / 3}
$$

with

$$
e\left(U_{t}, I_{t}\right) \geqslant\left(1+q-\left|I_{t-1}\right|^{-1 / 3}\right) e\left(U_{t-1}, I_{t-1}\right) \quad \text { or } \quad\left|I_{t}\right| \geqslant n / \log n
$$

and

$$
e\left(U_{t+1}, I_{t+1}\right)=\left(1+q \pm\left|I_{t}\right|^{-1 / 3}\right) e\left(U_{t}, I_{t}\right)
$$

we obtain the statement.

We continue with (b). Consider first the case $\left|I_{t}\right| \in[n / \log n, n / 2]$. Using Lemmas 2.7 and 2.8, i.e. $e\left(U_{t}, I_{t}\right) \geqslant \varepsilon\left|U_{t}\right|\left|I_{t}\right| \Delta_{n} / n+o\left(\Delta_{n}\right)\left|I_{t}\right|$, together with $\left|U_{t}\right| \geqslant n / 2$ implies

$$
\begin{aligned}
\mathbb{E}_{t}\left[\left|I_{t+1} \backslash I_{t}\right|\right] & =\sum_{u \in U_{t}} q \frac{\left|N(u) \cap I_{t}\right|}{|N(u)|} \\
& \geqslant \frac{q \cdot e\left(U_{t}, I_{t}\right)}{\Delta_{n}} \\
& \geqslant \frac{q \varepsilon\left|U_{t}\right|\left|I_{t}\right| \Delta_{n} / n+o\left(\Delta_{n}\right)\left|I_{t}\right|}{\Delta_{n}(1+o(1))} \\
& \geqslant\left(\frac{q \varepsilon}{2}+o(1)\right)\left|I_{t}\right| .
\end{aligned}
$$

Applying Lemma 2.5, where we set $g=2, f=\log n$ and $c=q \varepsilon / 2+o(1)$, we are finished with this part as well. Now let $\left|I_{t}\right| \in[n / 2, n-n / \log n]$. We switch our focus to the set of uninformed vertices. Using again the fact that $e\left(U_{t}, I_{t}\right) \geqslant \varepsilon\left|U_{t}\right|\left|I_{t}\right| \Delta_{n} / n+o\left(\Delta_{n}\right)\left|U_{t}\right|$, we have

$$
\begin{aligned}
\mathbb{E}_{t}\left[\left|U_{t+1}\right|\right] & =\sum_{u \in U_{t}} 1-q \frac{\left|N(u) \cap I_{t}\right|}{|N(u)|} \\
& =\sum_{u \in U_{t}} 1-q \frac{\left|N(u) \cap I_{t}\right|}{\Delta_{n}(1+o(1))} \\
& =\left|U_{t}\right|-\frac{q \cdot e\left(U_{t}, I_{t}\right)}{\Delta(1+o(1))} \\
& =\left|U_{t}\right|-\frac{q \varepsilon\left|U_{t}\right|\left|I_{t}\right| \Delta_{n} / n+o\left(\Delta_{n}\right)\left|U_{t}\right|}{\Delta_{n}(1+o(1))} \\
& \leqslant\left(1-\frac{q \varepsilon}{2}+o(1)\right)\left|U_{t}\right| .
\end{aligned}
$$

Inductively we obtain for any integer $\tau \geqslant 1$ the bound $\mathbb{E}_{t}\left[\left|U_{t+\tau}\right|\right] \leqslant(1-q \varepsilon / 2+o(1))^{\tau}\left|U_{t}\right|$, and so for some $\tau:=2 \log \log n / \log (1 /(1-q \varepsilon / 2+o(1)))=o(\log n)$ we have

$$
\mathbb{E}_{t}\left[\left|U_{t+\tau}\right|\right] \leqslant\left|U_{t}\right| / \log ^{2} n=o(n / \log n) .
$$

Hence, by Markov's inequality, $\mathbb{P}_{t}\left[\left|U_{t+\tau}\right| \geqslant n / \log n\right]=o(1)$.

In order to show (c), let $\left|I_{t}\right| \in[n-n / \log n, n]$. As for $q=1$ the term $1-q$ in Lemma 3.1(b) vanishes, we distinguish the cases $q=1$ and $q \neq 1$. We start with $q=1$. By induction, it follows that for any round $\tau>0$ and suitable $f=o(1)$,

$$
\mathbb{E}_{t}\left[\left|U_{t+\tau}\right|\right] \leqslant(f(n))^{\tau}\left|U_{t}\right|
$$


We choose $\tau=\log _{1 / f(n)}(n)=o(\log n)$ as $1 / f=\omega(1)$. Hence we obtain $\mathbb{E}_{t}\left[\left|U_{t+\tau}\right|\right] \leqslant\left|U_{t}\right| / n \leqslant$ $1 / \log n$. Therefore we have $\mathbb{P}_{t}\left[\left|U_{t+\tau}\right| \geqslant 1\right] \leqslant o(1)$ by Markov's inequality. For $q \neq 1$ we have by induction, for any number of rounds $\tau \geqslant 1$,

$$
\mathbb{E}_{t}\left[\left|U_{t+\tau}\right|\right] \leqslant(1-q+o(1))^{\tau}\left|U_{t}\right|
$$

We choose

$$
\tau=\log _{1 /(1-q+o(1))}(n)=-\log n / \log (1-q)+o(\log n) .
$$

Thus, using Markov's inequality, analogously to the case $q=1$, we obtain the desired upper bound.

Note that for $q=1$ this already implies Theorems 1.2(b) and 1.3(a). This leaves the case for $q \neq 1$.

Lemma 3.3. Let $0<\varepsilon \leqslant 1 / 2, q \in(0,1]$ and $\mathcal{G}=\left(G_{n}\right)_{n \in \mathbb{N}}$ be an expander sequence. Let $\tilde{\mathcal{G}}=$ $\left(\tilde{G}_{n}\right)_{n \in \mathbb{N}}$ be such that each $\tilde{G}_{n}$ is obtained by deleting edges of $G_{n}$, such that each vertex keeps at least $a(1 / 2+\varepsilon)$ fraction of its edges and abbreviate $I_{t}=I_{t}^{(\text {pull })}$. Let $q \in(0,1)$ and $\left|I_{t}\right| \leqslant n / 2$. Then, for $\tau=-\log n / \log (1-q)$ and all $c<1$, w.h.p. $\left|I_{t+c \tau}\right|<n$.

Proof. We consider a modified process in which vertices have a higher chance of getting informed. In particular, note that the probability that $u \in U_{t}$ gets informed is at most $q\left|N(u) \cap I_{t}\right| /|N(u)| \leqslant q$ and that all these events are independent; now we assume that each such $u$ gets independently informed with probability exactly $q$. Then the runtime in this modified model constitutes a lower bound for the runtime in the original model.

Let $c<1, u \in U_{t}$ and $E_{u}$ be the event that $u$ does not get informed in $c \tau$ rounds in this model. Thus

$$
\mathbb{P}\left[E_{u}\right]=(1-q)^{c \tau}=(1-q)^{-c \log n / \log (1-q)}=n^{-c}=\omega(1 / n)
$$

and as the events $E_{u}$ are independent and $\left|U_{t}\right|=\Theta(n)$,

$$
\mathbb{P}\left[\bigwedge_{u \in U_{t}} \overline{E_{u}}\right] \leqslant \prod_{u \in U_{t}} \mathbb{P}\left[\overline{E_{u}}\right] \leqslant \exp \left(-\sum_{u \in U_{t}} \mathbb{P}\left[E_{u}\right]\right)=o(1)
$$

\subsection{Proof of Theorem 1.2(c) - push\&pull is fast on expanders}

As we are now in the case without edge deletions, we begin with a lemma that determines the expected number of informed vertices in one round. Intuitively we will show that push and pull do not interact badly, and therefore pusherpull is given as a straightforward combination of push and pull.

Lemma 3.4. Let $\mathcal{G}$ be an expander sequence and abbreviate $I_{t}=I_{t}^{(p p)}$.

(a) Let $\left|I_{t}\right| \leqslant n / \log n$. Then $\mathbb{E}_{t}\left[\left|I_{t+1} \backslash I_{t}\right|\right]=(2 q+o(1))\left|I_{t}\right|$.

(b) Let $\left|U_{t}\right| \leqslant n / \log n$. Then $\mathbb{E}_{t}\left[\left|U_{t+1}\right|\right]=(1+o(1)) e^{-q}(1-q)\left|U_{t}\right|$. 
Proof. We begin with (a). The probability that $v \in U_{t}$ gets informed by pull is $q\left|N(v) \cap I_{t}\right| /|N(v)|$. Thus, using Lemma 2.7,

$$
\begin{aligned}
\mathbb{E}_{t}\left[\left|I_{t+1}^{(\text {pull })} \backslash I_{t}\right|\right] & =\sum_{u \in U_{t}} q \frac{\left|N(u) \cap I_{t}\right|}{|N(u)|} \\
& =q \sum_{u \in U_{t}} \frac{\left|N(u) \cap I_{t}\right|}{\Delta_{n}(1+o(1))} \\
& =(q+o(1)) \frac{e\left(U_{t}, I_{t}\right)}{\Delta_{n}} \\
& =(q+o(1)) \frac{\left|U_{t}\right|\left|I_{t}\right| \Delta_{n} / n+o\left(\Delta_{n}\right)\left|I_{t}\right|}{\Delta_{n}} .
\end{aligned}
$$

Since $\left|I_{t}\right|=o(n)$ we obtain that $\left|U_{t}\right|=(1-o(1)) n$, and this expression simplifies to $(q+o(1))\left|I_{t}\right|$.

Before we switch our attention to $p u s h$ we make a simple observation. Let $a_{1}, \ldots, a_{k}, k \in \mathbb{N}$ be real numbers. Then, using the fact that for any $a=o(1)$ it is $e^{-a+o(a)}=1-a$ and $e^{-a}=1-a+$ $o(a)$, we have

$$
\prod_{1 \leqslant i \leqslant k}\left(1-a_{i}\right)=\exp \left(-(1+o(1)) \sum_{1 \leqslant i \leqslant k} a_{i}\right)=1-(1+o(1)) \sum_{1 \leqslant i \leqslant k} a_{i} \quad \text { if } \sum_{1 \leqslant i \leqslant k} a_{i}=o(1) .
$$

The probability that $v \in U_{t}$ gets informed by push is

$$
1-\prod_{i \in N(v) \cap I_{t}}(1-q /|N(v)|) .
$$

According to Lemma 2.10(b) there is $B_{t} \subseteq U_{t}$ such that $\left|N(u) \cap I_{t}\right|=o(|N(u)|)$ for all $u \in B_{t}$ and $\left|U_{t} \backslash B_{t}\right|=o\left(\left|I_{t}\right|\right)$. Thus (3.3) is applicable, and in a similar fashion to (3.2) we get

$$
\begin{aligned}
\mathbb{E}_{t}\left[\left|I_{t+1}^{(\text {push })} \backslash I_{t}\right|\right] & =\sum_{u \in U_{t}} 1-\prod_{i \in N(u) \cap I_{t}}\left(1-\frac{q}{|N(i)|}\right) \\
& =q \sum_{u \in B_{t}} \frac{\left|N(u) \cap I_{t}\right|}{\Delta_{n}(1+o(1))}+o\left(\left|I_{t}\right|\right) \\
& =(q+o(1))\left|I_{t}\right| .
\end{aligned}
$$

We express the expected number of vertices informed by pushe $p$ pull after one additional round in terms of the expected values we just calculated ((3.2) and (3.4)):

$$
\begin{aligned}
\mathbb{E}_{t}\left[\left|I_{t+1} \backslash I_{t}\right|\right] & =\mathbb{E}_{t}\left[\left|I_{t+1}^{(\text {pull })} \backslash I_{t}\right|+\left|I_{t+1}^{(\text {push })} \backslash I_{t}\right|-\left|\left(I_{t+1}^{(\text {push })} \backslash I_{t}\right) \cap\left(I_{t+1}^{(\text {pull })} \backslash I_{t}\right)\right|\right] \\
& =(2 q-o(1))\left|I_{t}\right|-\mathbb{E}_{t}\left[\left|\left(I_{t+1}^{(\text {push })} \backslash I_{t}\right) \cap\left(I_{t+1}^{(\text {pull })} \backslash I_{t}\right)\right|\right] .
\end{aligned}
$$

Lemma 2.10(a) gives a set

$$
A_{t} \subseteq I_{t+1}^{(\text {push })},\left|A_{t}\right|=(1-o(1))\left|I_{t+1}^{(\text {push })}\right|
$$

such that

$$
\left|N(u) \cap I_{t+1}^{(p u s h)}\right|=o(1)|N(u)| \quad \text { for all } u \in A_{t} .
$$


Since push and pull happen independently,

$$
\begin{aligned}
\mathbb{E}_{t}\left[\left|\left(I_{t+1}^{\text {(pull })} \backslash I_{t}\right) \cap\left(I_{t+1}^{(\text {push })} \backslash I_{t}\right)\right| \mid I_{t+1}^{(\text {push })}\right] & =\sum_{u \in I_{t+1}^{\text {(push })} \backslash I_{t}} \mathbb{P}_{t}\left[u \in I_{t+1}^{(\text {pull })} \backslash I_{t}\right] \\
& =\sum_{u \in I_{t+1}^{(\text {push })} \backslash I_{t}} q \frac{\left|N(u) \cap I_{t}\right|}{|N(u)|} \\
& \leqslant \sum_{u \in A_{t}} q \frac{\left|N(u) \cap I_{t}\right|}{|N(u)|}+\sum_{u \in I_{t+1}^{(\text {push }} \backslash A_{t}} q \frac{\left|N(u) \cap I_{t}\right|}{|N(u)|} .
\end{aligned}
$$

Using the fact that $\left|N(u) \cap I_{t}\right|=o(|N(u)|)$ for all $u \in A_{t}$, we obtain

$$
\mathbb{E}_{t}\left[\left|\left(I_{t+1}^{(\text {pull })} \backslash I_{t}\right) \cap\left(I_{t+1}^{(\text {push })} \backslash I_{t}\right)\right|\right] \leqslant \mathbb{E}_{t}\left[o\left(\left|A_{t}\right|\right)+\left|I_{t+1}^{(\text {push })} \backslash A_{t}\right|\right]=o\left(\left|I_{t}\right|\right),
$$

as

$$
\left|A_{t}\right| \leqslant\left|I_{t+1}^{(\text {push })}\right| \leqslant 2\left|I_{t}\right| \text { and }\left|I_{t+1}^{(\text {push })} \backslash A_{t}\right|=o\left(\left|I_{t+1}^{(\text {push })}\right|\right)=o\left(\left|I_{t}\right|\right) .
$$

Combining this with (3.5) we get $\mathbb{E}_{t}\left[\left|I_{t+1} \backslash I_{t}\right|\right]=(2 q+o(1))\left|I_{t}\right|$, as claimed.

Next we show (b). Let $A_{u}$ be the event that an uninformed vertex $u$ does not get informed by the push algorithm, let $B_{u}$ be the corresponding event for pull. Then $A_{u}$ and $B_{u}$ are independent and $A_{u} \cap B_{u}$ is the event that $u$ does not get informed in the current round. We obtain

$$
\begin{aligned}
\mathbb{P}_{t}\left[A_{u}\right] & =\prod_{i \in N(u) \cap I_{t}}\left(1-\frac{q}{|N(i)|}\right) \\
& \leqslant\left(1-\frac{q}{\Delta_{n}}\right)^{\left|N(u) \cap I_{t}\right|} \\
& \leqslant \exp \left(-q \frac{\left|N(u) \cap I_{t}\right|}{\Delta_{n}}\right) \\
& =\exp \left(\frac{-q\left|N(u) \cap I_{t}\right|}{(1+o(1))|N(u)|}\right)
\end{aligned}
$$

and

$$
\mathbb{P}_{t}\left[B_{u}\right]=1-\frac{q\left|N(u) \cap I_{t}\right|}{|N(u)|} .
$$

According to Lemma 2.10(a) there is a set $C_{t} \subseteq U_{t},\left|C_{t}\right|=(1-o(1))\left|U_{t}\right|$ such that $\left|N(u) \cap I_{t}\right|=$ $(1-o(1))|N(u)|$ for all $u \in C_{t}$. As $\mathbb{P}_{t}\left[A_{u} \cap B_{u}\right] \leqslant 1$, we therefore get

$$
\mathbb{E}_{t}\left[\left|U_{t+1}\right|\right]=\sum_{u \in U_{t}} \mathbb{P}_{t}\left[A_{u} \cap B_{u}\right] \leqslant \sum_{u \in C_{t}} \mathbb{P}_{t}\left[A_{u}\right] \cdot \mathbb{P}_{t}\left[B_{u}\right]+\left|U_{t} \backslash C_{t}\right| \leqslant(1+o(1)) e^{-q}(1-q)\left|U_{t}\right|
$$

For the lower bound we need to find a lower bound on the probability of a single uninformed vertex not getting informed in one round by push. Indeed, for any $u \in U_{t}$ and sufficiently large $n$,

$$
\mathbb{P}_{t}\left[A_{u}\right]=\prod_{v \in N(u) \cap I_{t}}\left(1-\frac{q}{|N(v)|}\right) \geqslant\left(1-\frac{q}{\delta_{n}}\right)^{\left|N(u) \cap I_{t}\right|} \geqslant e^{-q \Delta_{n} / \delta_{n}} .
$$


Combining this inequality with the trivial bound $\mathbb{P}\left[B_{u}\right] \geqslant 1-q$, we get a lower bound on the expected number of uninformed vertices after one round using pusherpull:

$$
\begin{aligned}
\mathbb{E}_{t}\left[\left|U_{t+1}\right|\right] & =\sum_{u \in U_{t}} \mathbb{P}_{t}\left[A_{u} \cap B_{u}\right] \\
& =\sum_{u \in U_{t}} \mathbb{P}_{t}\left[A_{u}\right] \cdot \mathbb{P}_{t}\left[B_{u}\right] \\
& \geqslant e^{-q \Delta_{n} / \delta_{n}}(1-q)\left|U_{t}\right| \\
& =(1+o(1)) e^{-q}(1-q)\left|U_{t}\right|
\end{aligned}
$$

Next we show upper and lower bounds that together with Lemma 2.11 imply Theorem 1.2(c).

Lemma 3.5. Let $\mathcal{G}$ be an expander sequence and abbreviate $I_{t}=I_{t}^{(p p)}$. Let $q \in(0,1]$. Then the following statements hold w.h.p.

(a) Let $\sqrt{\log n} \leqslant\left|I_{t}\right| \leqslant n / \log n$. Then there are $\tau_{1}, \tau_{2}=\log _{1+2 q}\left(n /\left|I_{t}\right|\right)+o(\log n)$ such that $\left|I_{t+\tau_{2}}\right|<n / \log n<\left|I_{t+\tau_{1}}\right|$.

(b) Let $n / \log n \leqslant\left|I_{t}\right| \leqslant n-n / \log n$. Then there is $\tau=o(\log n)$ such that $\left|I_{t+\tau}\right|>n-n / \log n$.

(c) Let $\left|I_{t}\right| \geqslant n-n / \log n$.

(i) Case $q=1$. Then there is $\tau=o(\log n)$ such that $\left|I_{t+\tau}\right|=n$.

(ii) Case $q \neq 1$. Then there is $\tau \leqslant \log n /(q-\log (1-q))+o(\log n)$ such that $\left|I_{t+\tau}\right|=n$.

Proof. Since $\left|I_{t}\right| \geqslant\left|I_{t}^{(p u l l)}\right|$, statements (b) and (c) for $q=1$ follow immediately from Lemma 3.2. To see (a), note that by using Lemma 3.4 we get $\mathbb{E}_{t}\left[\left|I_{t+1} \backslash I_{t}\right|\right]=(2 q+o(1))\left|I_{t}\right|$, and applying Lemma 2.6 implies the claim.

Finally we show (c) for $q \neq 1$. Let $\left|I_{t}\right| \geqslant n-n / \log n$. By Lemma 3.4, we obtain that, for any $\tau \in \mathbb{N}$,

$$
\mathbb{E}_{t}\left[\left|U_{t+\tau}\right|\right]=\left((1+o(1)) e^{-q}(1-q)\right)^{\tau}\left|U_{t}\right|
$$

Thus we may choose $\tau=\log n /(q-\log (1-q))+o(\log n)$ such that, say, $\mathbb{E}_{t}\left[\left|U_{t+\tau}\right|\right] \leqslant\left|U_{t}\right| / n \leqslant$ $1 / \log n$. Thus $\mathbb{P}_{t}\left[\left|U_{t+\tau}\right| \geqslant 1\right] \leqslant o(1)$ by Markov's inequality.

Note that for $q=1$ this already implies Theorem 1.2(c). This leaves the case for $q \neq 1$.

Lemma 3.6. Let $\mathcal{G}$ be an expander sequence and abbreviate $I_{t}=I_{t}^{(p p)}$, let $q \in(0,1)$ and $\left|I_{t}\right| \leqslant n / 2$. Then for $\tau=\log n /(q-\log (1-q))$ and all $c<1$ w.h.p. $\left|I_{t+c \tau}\right|<n$.

Proof. We consider a modified process in which vertices have a higher chance of getting informed. In particular, note that the probability that $u \in U_{t}$ gets informed by pull is at most $q\left|N(u) \cap I_{t}\right| /|N(u)| \leqslant q$ and that all these events are independent; according to (3.6) the probability that $u \in U_{t}$ gets informed by push is at most $1-e^{-q \Delta_{n} / \delta_{n}}$. Now we assume that each such $u$ gets independently informed with probability exactly $1-e^{-q \Delta_{n} / \delta_{n}}(1-q)$. Then the runtime in this modified model constitutes a lower bound for the runtime in the original model. Let $u \in U_{t}$ and $E_{u}$ be the event that $u$ does not get informed in this modified model in $c \tau$ rounds. Thus, for $c<1$,

$$
\mathbb{P}\left[E_{u}\right] \geqslant\left((1-q) e^{-q \Delta_{n} / \delta_{n}}\right)^{c \tau}=\omega\left(n^{-1}\right),
$$


and as the events $E_{u}$ are independent and $\left|U_{t}\right|=\Theta(n)$,

$$
\mathbb{P}\left[\bigwedge_{u \in U_{t}} \overline{E_{u}}\right] \leqslant \prod_{u \in U_{t}} \mathbb{P}\left[\overline{E_{u}}\right] \leqslant \exp \left(-\sum_{u \in U_{t}} \mathbb{P}\left[E_{u}\right]\right)=o(1) .
$$

\subsection{Proof of Theorem 1.5(a) - push informs almost all vertices fast in spite of edge deletions}

To shorten the notation, let us call the setting with deleted edges the 'new model' and the setting without deleted edges the 'old model', that is, the term 'new model' corresponds to the graphs in $\tilde{\mathcal{G}}$ while 'old model' refers to the (original) graphs in $\mathcal{G}$. We prove Lemma 3.7, which directly implies Theorem 1.5(a). We write $I_{t}=I_{t}^{(\text {push })}$ throughout.

Lemma 3.7. Under the assumptions of Theorem 1.5(a), the following holds for the new model:

(a) There are $\tau, \tilde{\tau}=\log _{1+q}(n)+o(\log n)$ such that w.h.p. $\left|I_{\tilde{\tau}}\right|<n / \log n<\left|I_{\tau}\right|$.

(b) Assume $\left|I_{t}\right| \geqslant n / \log n$. Then there is a $\tau=o(\log n)$ such that w.h.p. $\left|I_{t+\tau}\right| \geqslant n-n / \log n$.

For the proof of Lemma 3.7 we will need the following statements, the first one taken from [28].

Lemma 3.8 (proof of Lemma 2.5 in [28]). Consider the old model. Assume $\left|I_{t}\right|<n / \log n$ and $q=1$. Then

$$
\mathbb{P}_{t}\left[\left|I_{t+1}\right|=\left|I_{t}\right|+(1-o(1))\left|I_{t}\right|\right]=1-o(1) .
$$

Lemma 3.9. Consider push on a sequence of graphs $\left(G_{n}\right)_{n \in \mathbb{N}}$, where $G_{n}$ has $n$ vertices. Assume that $\left|I_{t}\right|=\omega(1)$ and that (3.7) holds for $q=1$, that is, assume that

$$
\mathbb{P}_{t}\left[\left|I_{t+1}\right|=\left|I_{t}\right|+(1-o(1))\left|I_{t}\right|\right]=1-o(1) \quad \text { for } q=1 .
$$

Then, for $q \in(0,1]$,

$$
\mathbb{P}_{t}\left[\left|I_{t+1}\right|=\left|I_{t}\right|+(q-o(1))\left|I_{t}\right|\right]=1-o(1) .
$$

Moreover, assume that whenever $\left|I_{t}\right|<n / \log n$, for $q=1$, (3.7) holds. Then there are $\tau, \tilde{\tau}=$ $\log _{1+q}(n)+o(\log n)$ such that w.h.p.

$$
\left|I_{\tilde{\tau}}\right|<n / \log n<\left|I_{\tau}\right| .
$$

Proof. For a graph $G$ and for $v \in I_{t}$, let $X_{v}(G)$ denote the vertex to which $v$ pushes in round $t$. Let

$$
N_{t+1}:=\left\{X_{v}\left(G_{n}\right) \mid v \in I_{t}\right\} \cap U_{t} .
$$

Note that whenever $\left|I_{t}\right|<n / \log n$, w.h.p. $\left|N_{t+1}\right|=(1-o(1))\left|I_{t}\right|$ from (3.7). For $q \in(0,1]$ each vertex in $N_{t+1}$ has a probability at least $q$ of being informed and all these events are independent; thus (3.8) follows directly by applying the Chernoff bounds whenever $\left|I_{t}\right|=\omega(1)$.

In order to prove the second statement we call a round $t$ that does not satisfy (3.8) a failed round. Note that we just argued that the probability that a round fails is $o(1)$ whenever $\left|I_{t}\right|=$ $\omega(1)$ and $\left|I_{t}\right|<n / \log n$, and the events that distinct rounds fail are independent. In particular, the number of failed rounds among the next $R$ rounds, assuming that $\left|I_{t}\right|$ stays below $n / \log n$, is w.h.p. $o(R)$. Moreover, if a round does not fail, the number of informed vertices increases by a factor of $(1+q+o(1))$ and otherwise it may increase by an arbitrary factor in the interval $[1,2]$. 
Finally, Lemma 2.11 yields that there is $t^{*}=o(\log n)$ such that w.h.p. $\left|I_{t^{*}}\right|=\omega(1)$, which implies that after $R+t^{*}$ rounds, the number of informed vertices is w.h.p. in the interval

$$
\left[(1+q+o(1))^{R-o(R)},(1+q+o(1))^{R-o(R)} \cdot 2^{o(R)}\right],
$$

and choosing $R=\log _{1+q}(n)+o(\log n)$ in two ways establishes (3.9).

In the subsequent proof of Lemma 3.7 we will use the simple observations that, for any $n \in \mathbb{N}_{0}$,

$$
\mathbb{P}[\operatorname{Bin}(n, 1 / 2) \geqslant n / 2] \geqslant 1 / 2 \text { and } \mathbb{P}[\operatorname{Bin}(n, 1 / 4) \geqslant n / 4] \geqslant 1 / 4
$$

(see e.g. [22] when $n>4$ ), and the other cases are checked easily.

Proof of Lemma 3.7. We first show (a). We assume $q=1$ and prove that, for $\left|I_{t}\right|<n / \log n$, (3.7) also holds in the new model; then claim (a) follows directly from Lemma 3.9. Let $G=(V, E)$ be a graph. For $v \in I_{t}$ let $X_{v}(G)$ denote the vertex to which $v$ pushes in round $t$. For $u \in V$ let $c_{u}(G):=$ $\left|\left\{v \in I_{t} \mid X_{v}(G)=u\right\}\right|$ denote the number of times $u$ is pushed in round $t$. Let

$$
\mathcal{Y}_{t}(G):=\left\{v \in I_{t} \mid c_{v}(G)=1\right\} \quad \text { and } \quad \mathcal{H}_{t}(G):=\left\{v \in I_{t} \mid c_{v}(G) \geqslant 1\right\}
$$

denote the set of informed vertices that are being pushed exactly once in round $t$ and the set of informed vertices that are being pushed at least once in round $t$ respectively. Let

$$
\mathcal{Z}_{t}(G):=\left\{v \in V \mid c_{v}(G) \geqslant 2\right\}
$$

denote the set of vertices that are being pushed more than once in round $t$. Let $Y_{t}(G):=\left|\mathcal{Y}_{t}(G)\right|$ and $H_{t}(G):=\left|\mathcal{H}_{t}(G)\right|$ and, in a slight abuse of notation, let

$$
Z_{t}(G):=\sum_{k \geqslant 2}(k-1) \cdot\left|\left\{v \in V \mid c_{v}(G)=k\right\}\right|
$$

denote the number of vertices that are being pushed multiple times in round $t$ counted with multiplicity. Note that the quantity $Y+Z$ denotes the number of pushes that have no effect in the respective round, that is, there are $Y+Z$ pushes that are useless in the sense that even without them, the same number of vertices would become informed in the respective round. In the following paragraphs we condition on $I_{t}$ implicitly, that is, we write $\mathbb{P}[\ldots]$ instead of $\mathbb{P}_{t}[\ldots]$, etc., to lighten the notation. We want to show that (3.7) does hold in the new model; for contradiction we assume that this is not the case. Hence we can infer that there is a constant $c>0$ such that

$$
\limsup _{n \rightarrow \infty} \mathbb{P}\left[Y_{t}\left(\tilde{G}_{n}\right) \geqslant c\left|I_{t}\right|\right]>0 \quad \text { or } \quad \limsup _{n \rightarrow \infty} \mathbb{P}\left[Z_{t}\left(\tilde{G}_{n}\right) \geqslant c\left|I_{t}\right|\right]>0 .
$$

Thus, without loss of generality, we can assume that there is $f^{*}>0$ and $n_{0} \in \mathbb{N}$ such that

$$
\mathbb{P}\left[Y_{t}\left(\tilde{G}_{n}\right) \geqslant c\left|I_{t}\right|\right]>f^{*} \text { for all } n \geqslant n_{0} \quad \text { or } \quad \mathbb{P}\left[Z_{t}\left(\tilde{G}_{n}\right) \geqslant c\left|I_{t}\right|\right]>f^{*} \text { for all } n \geqslant n_{0} .
$$

If this is not the case we can restrict ourselves to a suitable subsequence of $(n)_{n \in \mathbb{N}}$ on which it is true. Next, we describe an explicit coupling between the new and the old model. For any vertex $v$ consider $X_{v}\left(G_{n}\right)$. If $X_{v}\left(G_{n}\right) \in N_{\tilde{G}_{n}}(v)$, then set $X_{v}\left(\tilde{G}_{n}\right):=X_{v}\left(G_{n}\right)$ and otherwise choose $X_{v}\left(\tilde{G}_{n}\right)$ uniformly at random from $N_{\tilde{G}_{n}}(v)$. Note that $X_{v}\left(G_{n}\right), X_{v}\left(\tilde{G}_{n}\right)$ have by construction the correct marginal distribution. Moreover, note that by construction, the family

$$
\left(X_{v}\left(G_{n}\right) \mid\left(X_{u}\left(\tilde{G}_{n}\right)\right)_{u \in V_{n}}\right)_{v \in V_{n}}
$$

of random variables is independent, since $X_{v}\left(G_{n}\right)$ depends only on $X_{v}\left(\tilde{G}_{n}\right)$ for all $v \in V_{n}$. 
We begin with the case that $\mathbb{P}\left[Y_{t}\left(\tilde{G}_{n}\right) \geqslant c\left|I_{t}\right|\right]>f^{*}$. We will show

$$
\mathbb{P}\left[H_{t}\left(G_{n}\right) \geqslant Y_{t}\left(\tilde{G}_{n}\right) / 2 \mid \mathcal{Y}_{t}\left(\tilde{G}_{n}\right)\right] \geqslant 1 / 2
$$

and then, since by assumption $\mathbb{P}\left[Y_{t}\left(\tilde{G}_{n}\right) \geqslant c\left|I_{t}\right|\right]>f^{*}$, we can infer $\mathbb{P}\left[H_{t}\left(G_{n}\right) \geqslant c\left|I_{t}\right| / 2\right] \geqslant f^{*} / 2$, which contradicts Lemma 3.8. Let $\mathcal{Y}_{t}\left(\tilde{G}_{n}\right)=\left\{y_{1}, \ldots, y_{Y_{t}\left(\tilde{G}_{n}\right)}\right\}$. Then there are distinct vertices $v_{1}, \ldots, v_{Y_{t}\left(\tilde{G}_{n}\right)} \in I_{t}$ such that $X_{v_{i}}\left(\tilde{G}_{n}\right)=y_{i}$ for all $i \in\left\{1, \ldots, Y_{t}\left(\tilde{G}_{n}\right)\right\}$. Due to (3.11) the events $\left(\left\{X_{v_{i}}\left(G_{n}\right)=X_{v_{i}}\left(\tilde{G}_{n}\right)\right\}\right)_{1 \leqslant i \leqslant Y_{t}}$ are independent. Moreover, for all $i \in\left\{1, \ldots, Y_{t}\left(\tilde{G}_{n}\right)\right\}$,

$$
\mathbb{P}\left[X_{v_{i}}\left(G_{n}\right)=X_{v_{i}}\left(\tilde{G}_{n}\right) \mid \mathcal{Y}_{t}\left(\tilde{G}_{n}\right)\right]=\frac{d_{\tilde{G}_{n}}\left(v_{i}\right)}{d_{G_{n}}\left(v_{i}\right)} \geqslant 1 / 2+\varepsilon
$$

and therefore, given $\mathcal{Y}_{t}\left(\tilde{G}_{n}\right), H_{t}\left(G_{n}\right)$ dominates a binomially distributed random variable $\operatorname{Bin}\left(Y_{t}\left(\tilde{G}_{n}\right), 1 / 2\right)$. In particular, this implies with (3.10) that

$$
\mathbb{P}\left[H_{t}\left(G_{n}\right) \geqslant Y_{t}\left(\tilde{G}_{n}\right) / 2 \mid \mathcal{Y}_{t}\left(\tilde{G}_{n}\right)\right] \geqslant 1 / 2,
$$

as claimed.

We continue with the case $\mathbb{P}\left[Z_{t}\left(\tilde{G}_{n}\right) \geqslant c\left|I_{t}\right|\right]>f^{*}$. Let $\mathcal{Z}_{t}\left(\tilde{G}_{n}\right)=\left\{z_{1}, \ldots, z_{\left|\mathcal{Z}_{t}\left(\tilde{G}_{n}\right)\right|}\right\}$. Then, for any $i \in\left\{1, \ldots,\left|\mathcal{Z}_{t}\left(\tilde{G}_{n}\right)\right|\right\}$ let $n_{i}:=c_{z_{i}}\left(\tilde{G}_{n}\right) \geqslant 2$, that is, there are distinct vertices $v_{i, 1}, \ldots, v_{i, n_{i}}$ such that $X_{v}\left(\tilde{G}_{n}\right)=z_{i}$ for all $v \in\left\{v_{i, 1}, \ldots, v_{i, n_{i}}\right\}$. We will show that

$$
\mathbb{P}\left[Z_{t}\left(G_{n}\right) \geqslant Z_{t}\left(\tilde{G}_{n}\right) / 8 \mid \mathcal{Z}_{t}\left(\tilde{G}_{n}\right), n_{1}, \ldots, n_{\left|\mathcal{Z}_{t}\left(\tilde{G}_{n}\right)\right|}\right] \geqslant 1 / 8
$$

and then, since by assumption $\mathbb{P}\left[Z_{t}\left(\tilde{G}_{n}\right) \geqslant c\left|I_{t}\right|\right]>f^{*}$, we obtain $\mathbb{P}\left[Z_{t}\left(G_{n}\right) \geqslant c / 8\left|I_{t}\right|\right] \geqslant f^{*} / 8$, which contradicts Lemma 3.8. Due to (3.11) the events

$$
\left(\left\{X_{v_{i, j}}\left(G_{n}\right)=X_{v_{i, j}}\left(\tilde{G}_{n}\right)\right\}\right)_{1 \leqslant i \leqslant\left|\mathcal{Z}_{t}\left(\tilde{G}_{n}\right)\right|, 1 \leqslant j \leqslant n_{i}}
$$

are independent. Moreover, for all $1 \leqslant i \leqslant\left|\mathcal{Z}_{t}\left(\tilde{G}_{n}\right)\right|, 1 \leqslant j \leqslant n_{i}$,

$$
\mathbb{P}\left[X_{v_{i, j}}\left(G_{n}\right)=X_{v_{i, j}}\left(\tilde{G}_{n}\right) \mid \mathcal{Z}_{t}\left(\tilde{G}_{n}\right), n_{1}, \ldots, n_{\left|\mathcal{Z}_{t}\left(\tilde{G}_{n}\right)\right|}\right]=\frac{d_{\tilde{G}_{n}}\left(v_{i, j}\right)}{d_{G_{n}}\left(v_{i, j}\right)} \geqslant 1 / 2+\varepsilon .
$$

For $1 \leqslant i \leqslant\left|\mathcal{Z}_{t}\left(\tilde{G}_{n}\right)\right|$ let $B_{i} \sim \operatorname{Bin}\left(n_{i}, 1 / 2\right)$ be independent random variables. Moreover, let $M_{1}:=$ $\left\{i|1 \leqslant i \leqslant| \mathcal{Z}_{t}\left(\tilde{G}_{n}\right) \mid, n_{i}=2\right\}$ and $M_{2}:=\left\{i|1 \leqslant i \leqslant| \mathcal{Z}_{t}\left(\tilde{G}_{n}\right) \mid, n_{i}>2\right\}$. Using (3.13) and (3.14), given $\mathcal{Z}_{t}\left(\tilde{G}_{n}\right), n_{1}, \ldots, n_{\left|\mathcal{Z}_{t}\left(\tilde{G}_{n}\right)\right|}$, we infer that $Z_{t}\left(G_{n}\right)$ dominates

$$
\sum_{i=1}^{\left|\mathcal{Z}_{t}\left(\tilde{G}_{n}\right)\right|} \max \left\{B_{i}-1,0\right\} \geqslant \sum_{i \in M_{1}} \max \left\{B_{i}-1,0\right\}+\sum_{i \in M_{2}} B_{i}-\left|M_{2}\right| .
$$

We treat the two sums individually. Note that $\sum_{i \in M_{1}} \max \left\{B_{i}-1,0\right\} \sim \operatorname{Bin}\left(\left|M_{1}\right|, 1 / 4\right)$; in particular,

$$
\mathbb{P}\left[\sum_{i \in M_{1}} \max \left\{B_{i}-1,0\right\} \geqslant\left|M_{1}\right| / 4\right] \geqslant 1 / 4
$$

by (3.10). Regarding the second sum, since $\sum_{i \in M_{2}} B_{i} \sim \operatorname{Bin}\left(\sum_{i \in M_{2}} n_{i}, 1 / 2\right)$, we obtain

$$
\mathbb{P}\left[\sum_{i \in M_{2}} B_{i} \geqslant 1 / 2 \sum_{i \in M_{2}} n_{i}\right] \geqslant 1 / 2
$$


Thus, given $\mathcal{Z}_{t}\left(\tilde{G}_{n}\right), n_{1}, \ldots, n_{\left|\mathcal{Z}_{t}\left(\tilde{G}_{n}\right)\right|}$ and using $2\left|M_{1}\right|=\sum_{i \in M_{1}} n_{i}$ and $\sum_{i \in M_{2}} n_{i} \geqslant 3\left|M_{2}\right|$, we infer that with probability at least $1 / 4 \cdot 1 / 2=1 / 8$

$$
\begin{aligned}
Z_{t}\left(G_{n}\right) & \geqslant \frac{1}{4}\left|M_{1}\right|+\frac{1}{2} \sum_{i \in M_{2}} n_{i}-\left|M_{2}\right| \\
& =\frac{1}{8} \sum_{i \in M_{1}} n_{i}+\frac{1}{2} \sum_{i \in M_{2}} n_{i}-\left|M_{2}\right| \\
& \geqslant \frac{1}{8} \sum_{i \in M_{1}} n_{i}+\frac{1}{6} \sum_{i \in M_{2}} n_{i} \\
& \geqslant \frac{1}{8} \sum_{i=1}^{\left|\mathcal{Z}_{t}\left(\tilde{G}_{n}\right)\right|} n_{i} \\
& =\frac{1}{8}\left(Z_{t}\left(\tilde{G}_{n}\right)+\left|\mathcal{Z}_{t}\left(\tilde{G}_{n}\right)\right|\right) \\
& \geqslant \frac{1}{8} Z_{t}\left(\tilde{G}_{n}\right) .
\end{aligned}
$$

This establishes (3.12). All in all, for $q=1$ we have shown that (3.7) also holds in the new model. Hence claim (a) follows directly from Lemma 3.9.

Next we prove claim (b). We write $\Delta_{n}:=\Delta\left(G_{n}\right), \tilde{\Delta}_{n}:=\Delta\left(\tilde{G}_{n}\right), \delta_{n}:=\delta\left(G_{n}\right)$ and $\tilde{\delta}_{n}:=\delta\left(\tilde{G}_{n}\right)$; moreover, we write $\tilde{N}(\cdot)$ instead of $N_{\tilde{G}_{n}}(\cdot)$. We assume that $\left|I_{t}\right| \in[n / \log n, n-n / \log n]$. We further distinguish two cases, namely $\left|I_{t}\right| \in[n / \log n, n / 2]$ and $\left|I_{t}\right| \in[n / 2, n-n / \log n]$. We start with the case $\left|I_{t}\right| \in[n / \log n, n / 2]$. Using Lemmas 2.7 and 2.8 and the assumption that $\Delta_{n} / \delta_{n}=$ $1+o(1)$ we obtain, for any $0<\bar{\varepsilon}<\varepsilon / 2$, for $n$ sufficiently large,

$$
e\left(I_{t}, U_{t}\right)>\bar{\varepsilon} \delta_{n}\left|I_{t}\right| .
$$

Using the fact that $e^{x} \geqslant(1+x / n)^{n}$ for $n \in \mathbb{N}$ and $|x| \leqslant n$, we obtain

$$
\mathbb{E}_{t}\left[\left|I_{t+1} \backslash I_{t}\right|\right] \geqslant \sum_{u \in \tilde{N}\left(I_{t}\right) \backslash I_{t}}\left[1-\prod_{v \in \tilde{N}(u) \cap I_{t}}\left(1-\frac{q}{\tilde{\Delta}_{n}}\right)\right] \geqslant \sum_{u \in \tilde{N}\left(I_{t}\right) \backslash I_{t}} 1-e^{-\left|\tilde{N}(u) \cap I_{t}\right| q / \tilde{\Delta}_{n}} .
$$

Further, using the fact that $e^{-x} \leqslant 1-x / 2$ for any $x \in(0,1)$ and (3.15) yields the bound

$$
\mathbb{E}_{t}\left[\left|I_{t+1} \backslash I_{t}\right|\right] \geqslant \sum_{u \in \tilde{N}\left(I_{t}\right) \backslash I_{t}} \frac{q\left|\tilde{N}(u) \cap I_{t}\right|}{2 \tilde{\Delta}_{n}}=\frac{q e\left(I_{t}, U_{t}\right)}{2 \tilde{\Delta}_{n}} \geqslant \frac{\bar{\varepsilon} q \delta_{n}}{2 \Delta_{n}}\left|I_{t}\right| .
$$

For this case the claim follows by Lemma 2.5, when setting $f=n / \log n, g=\log n$ and $c=$ $\bar{\varepsilon} q \delta_{n} /\left(2 \Delta_{n}\right)$.

Finally we consider the case $\left|I_{t}\right| \in[n / 2, n-n / \log n]$; here we examine the shrinking of $U_{t}$. Using Lemmas 2.7 and 2.8 we obtain, for any $0<\bar{\varepsilon}<\varepsilon / 2$, for $n$ sufficiently large, $e\left(I_{t}, U_{t}\right)>$ $\bar{\varepsilon} \delta_{n}\left|U_{t}\right|$. Hence, again using the fact that for any $x \in(0,1)$ it holds that $e^{-x} \leqslant 1-x / 2$, and that for $n \in \mathbb{N}$ and $|x| \leqslant n$ it is $e^{x} \geqslant(1+x / n)^{n}$, we obtain

$$
\begin{aligned}
\mathbb{E}_{t}\left[\left|U_{t+1}\right|\right] & =\sum_{u \in U_{t}} \prod_{v \in \tilde{N}(u) \cap I_{t}}\left(1-\frac{q}{d_{\tilde{G}_{n}}(v)}\right) \\
& \leqslant \sum_{u \in U_{t}} e^{-\left|\tilde{N}(u) \cap I_{t}\right| q / \tilde{\Delta}_{n}}
\end{aligned}
$$




$$
\begin{aligned}
& \leqslant \sum_{u \in U_{t}} 1-\frac{q\left|\tilde{N}(u) \cap I_{t}\right|}{2 \tilde{\Delta}_{n}} \\
& \leqslant\left|U_{t}\right|-\frac{\bar{\varepsilon} q \delta_{n}}{2 \tilde{\Delta}_{n}}\left|U_{t}\right| \\
& \leqslant\left(1-\frac{\bar{\varepsilon} q \delta_{n}}{2 \Delta_{n}}\right)\left|U_{t}\right| .
\end{aligned}
$$

Using the tower property of conditional expectation, we immediately get

$$
\mathbb{E}_{t}\left[\left|U_{t+\tau}\right|\right] \leqslant\left(1-\frac{\bar{\varepsilon} q \delta_{n}}{2 \Delta_{n}}\right)^{\tau}\left|U_{t}\right|, \quad \tau \in \mathbb{N}
$$

Thus, for

$$
\tau:=-2 \log \log (n) / \log \left(1-\bar{\varepsilon} q \delta_{n} /\left(2 \Delta_{n}\right)\right)=o(\log n),
$$

we have $\mathbb{E}_{t}\left[\left|U_{t+\tau}\right|\right]=o(n / \log n)$. Hence by Markov's inequality, $\mathbb{P}\left[\left|U_{t+\tau}\right| \geqslant n / \log n\right]=o(1)$.

\subsection{Proof of Theorem 1.4(a) - edge deletions slow down push}

Let $I_{t}^{(p u s h)}:=I_{t}$. In order to show the claim we construct an explicit sequence of graphs that has the desired property. More precisely, for any $\varepsilon>0$, each $q \in(0,1]$ and $n \in \mathbb{N}$ we will define a graph $G_{n}(\varepsilon)$ that is obtained by deleting edges from the complete graph on $n$ vertices such that each vertex keeps at least a $(1-\varepsilon)$ fraction of its edges and such that push slows down significantly.

We define $G_{n}(\varepsilon)=\left(V_{1} \cup V_{2}, E\right)$ with vertex set $V=V_{1} \cup V_{2}$, where $V_{1}:=\{1, \ldots,\lfloor n / 2\rfloor\}$ and $V_{2}:=\{\lfloor n / 2\rfloor+1, \ldots, n\}$, as follows. We include in $E$ all pairs of vertices that intersect $V_{1}$ and, moreover, we add edges (that now have endpoints only in $V_{2}$ ) such that all vertices in $V_{2}$ have degree $\lceil(1-\varepsilon) n\rceil+1 \pm 1$. According to Lemma 3.7(a) there is a $t=\log _{1+q}(n)+o(\log n)$ such that w.h.p. $\left|I_{t}\right|<n / \log n$. It thus suffices to show that it takes w.h.p. at least $(1+\varepsilon / 2) q^{-1} \log n$ more rounds to inform all remaining vertices.

Let $U_{t}^{\prime}:=U_{t}^{(\text {push })} \cap V_{2}$. As $\left|I_{t}\right|<n / \log n$ we have $\left|U_{t}^{\prime}\right| \geqslant n / 4$ with plenty of room to spare. In the remainder of this proof we will consider a modified process in which vertices have a higher chance of getting informed; in particular we assume that in each round, all vertices choose a neighbour independently and uniformly at random and after this round the chosen vertices are informed. Let $E_{u}$ denote the event that $u \in U_{t}^{\prime}$ does not get informed within the next $\tau:=(1+\varepsilon / 2) q^{-1} \log n$ rounds in this modified model. Each vertex $u \in U_{t}^{\prime}$ has $\lfloor n / 2\rfloor$ neighbours that have degree $n-1$, at most $\lceil(1-\varepsilon) n\rceil+1 \pm 1-\lfloor n / 2\rfloor \leqslant(1 / 2-\varepsilon) n+4$ neighbours that have at least degree $(1-\varepsilon) n$ and no further neighbours. Therefore, using the fact that for any $a \in \mathbb{R}$ we have $(1+a / n)^{n}=$ $e^{a}+O(1 / n)$, we obtain for each $u \in U_{t}^{\prime}$

$$
\begin{aligned}
\mathbb{P}_{t}\left[E_{u}\right] & \geqslant\left(\left(1-\frac{q}{n-1}\right)^{n / 2}\left(1-\frac{q}{(1-\varepsilon) n}\right)^{(1 / 2-\varepsilon) n+4}\right)^{\tau} \\
& =(1+o(1))\left(e^{-q(1 / 2+(1 / 2-\varepsilon) /(1-\varepsilon))}\right)^{\tau} \\
& =(1+o(1)) \exp \left(-\frac{4-4 \varepsilon-3 \varepsilon^{2}}{4-4 \varepsilon} \log n\right) \\
& =\omega\left(n^{-1}\right) .
\end{aligned}
$$

In this modified model the events $\left\{\overline{E_{u}} \mid u \in U_{t}^{\prime}\right\}$ also satisfy $\mathbb{P}_{t}\left[\overline{E_{u}} \mid\left\{\overline{E_{v}}: v \in U\right\}\right] \leqslant 1-p$ for all $u \in V_{2}$ and $U \subseteq V \backslash\{u\}$ and for some $p=\omega\left(n^{-1}\right)$. This follows immediately from the previous 
calculation, as conditioning on an event like $\left\{\overline{E_{v}}: v \in U\right\}$ only decreases the number of vertices that can push to $u$. Thus, as $\left|U_{t}^{\prime}\right|=\Theta(n)$,

$$
\mathbb{P}_{t}\left[\bigwedge_{u \in U_{t}^{\prime}} \overline{E_{u}}\right] \leqslant \prod_{u \in U_{t}^{\prime}}(1-p) \leqslant \exp \left(-\sum_{u \in U_{t}^{\prime}} p\right)=o(1)
$$

\subsection{Proof of Theorems 1.3(b) and 1.5(a) - push\&pull informs almost all vertices fast in spite of edge deletions}

Before we show the actual proof we will first present an informal argument that contains all relevant ideas and important observations. Let $\sqrt{\log n} \leqslant\left|I_{t}\right| \leqslant n / \log n$ and assume $q=1$. In Section 3.3 we proved that for push the informed vertices nearly double in every round for an arbitrary expander sequence with edge deletions and an otherwise arbitrary set $I_{t}$. For pull this is not true; however, we proved in Section 3.1 that the number of edges between the informed and the uninformed vertices nearly doubles in every round. The first attempt towards the proof of Theorems 1.3(b) and 1.5(b) then seems obvious: one would try to show that either the vertices triple every round, or the the edges do so, or for example that the product of the two quantities increases by a factor of 9. As it turns out, this is in general not the case; indeed, it is possible to choose an expander sequence, to delete edges such that each vertex keeps at least an $(1 / 2+\varepsilon)$ fraction of its neighbours, and to choose a (large) set of informed vertices $I_{t}$ such that after one round w.h.p. either $\left|I_{t+1}\right|<c\left|I_{t}\right|$ or $e\left(I_{t+1}, U_{t+1}\right)<c e\left(I_{t}, U_{t}\right)$ or $\left|I_{t+1}\right| e\left(I_{t+1}, U_{t+1}\right)<c^{2}\left|I_{t}\right| e\left(I_{t}, U_{t}\right)$ for some $c<3$. On the other hand, and although we have no explicit description of these 'malicious' sets, it seems rather unlikely that such sets will occur several times during the execution of pusherpull.

In order to show the claimed running time of pusherpull we will impose some additional structure. Let $\varepsilon>0$. In the subsequent exposition we assume that our graph $G$ - obtained from an expander by deleting edges such that each vertex keeps at least a $(1 / 2+\varepsilon)$ fraction of the edges has a very special structure. In particular, we assume that there is a partition $\Pi=\left(V_{i}\right)_{i \in[k]}$ of the vertex set of $G$ into a bounded number $k$ of equal parts such that $E_{G}\left(V_{i}\right)=\emptyset$ for all $1 \leqslant i \leqslant k$ and such that the induced subgraph $\left(V_{i}, V_{j}\right)$ looks like a random regular bipartite graph for all $1 \leqslant i<j \leqslant k$. Of course, not every relevant $G$ admits such a partition; however, Szemerédi's Regularity Lemma guarantees that every sufficiently large graph has a partition that is in a welldefined sense almost like the one described previously, and a substantial part of our proof is concerned with showing that being 'almost special' does not hurt significantly.

Assuming that $G$ is very special, let us collect some easy facts. Denote the degree of $u \in V_{i}$ in the induced subgraph $\left(V_{i}, V_{j}\right)$ by $d_{i j}$; this immediately gives $d_{G}(u)=\sum_{\ell=1}^{k} d_{i \ell}$, and note that $d_{i i}=0$ as there are no edges in $V_{i}$. Moreover, regular bipartite random graphs satisfy an expander property, that is,

$$
\begin{aligned}
e\left(W_{i}, W_{j}\right) & =d_{i, j}\left|W_{i}\right|\left|W_{j}\right| /\left|V_{j}\right|+o\left(d_{i, j}\right)\left|W_{i}\right| \\
& \approx\left|W_{i}\right|\left|W_{j}\right| d_{i j} k / n \text { for all } W_{i} \subseteq V_{i}, W_{j} \subseteq V_{j}, 1 \leqslant i<j \leqslant k,
\end{aligned}
$$

where we used the fact that all $\left|V_{i}\right|$ are of equal size. This is quite similar to the property that we used in our preceding analysis on expander sequences; see Lemma 2.7. As a pair in $\Pi$ behaves like a bipartite expander sequence, we can easily compute the expected number of informed vertices like we did in Section 3.2. We do so now for pull. Let $\left|I_{t+1}^{i, j}\right|$ be the number of vertices in $V_{i}$ informed after round $t+1$ by pull from vertices only in $V_{j}$ and set $I_{t}^{i}:=I_{t} \cap V_{i}, U_{t}^{i}:=U_{t} \cap V_{i}$ for all $1 \leqslant i \leqslant k$. Thus, as long as $I_{t}^{i}$ is much smaller than $V_{i}$ (and thus also $U_{t}^{i} \approx\left|V_{i}\right|=n / k$ ), we get 


$$
\mathbb{E}_{t}\left[\left|I_{t+1}^{(p u l l), i, j} \backslash I_{t}\right|\right]=\sum_{u \in U_{t}^{i}} \frac{\left|N(u) \cap I_{t}^{j}\right|}{d(u)}=\frac{e\left(U_{t}^{i}, I_{t}^{j}\right)}{\sum_{1 \leqslant \ell \leqslant k} d_{i \ell}} \approx \frac{d_{i j}}{\sum_{1 \leqslant \ell \leqslant k} d_{i \ell}}\left|I_{t}^{j}\right| .
$$

A similar calculation, which we do not perform in detail, yields for push

$$
\mathbb{E}_{t}\left[\left|I_{t+1}^{(p u s h), i, j} \backslash I_{t}\right|\right] \approx \frac{d_{i j}}{\sum_{1 \leqslant \ell \leqslant k} d_{\ell j}}\left|I_{t}^{j}\right|
$$

Moreover, as in previous proofs it turns out that the number of vertices informed simultaneously by push as well as pull is negligible; compare with the proof of Lemma 3.4. Thus we obtain that more or less

$$
\mathbb{E}_{t}\left[\left|I_{t+1}^{(p p), i, j}\right|\right] \approx\left|I_{t}^{i}\right|+\left(\frac{d_{i j}}{\sum_{1 \leqslant \ell \leqslant k} d_{i \ell}}+\frac{d_{i j}}{\sum_{1 \leqslant \ell \leqslant k} d_{\ell j}}\right)\left|I_{t}^{j}\right|,
$$

and by linearity of expectation

$$
\mathbb{E}_{t}\left[\left|I_{t+1}^{(p p), i}\right|\right] \approx\left|I_{t}^{i}\right|+\sum_{1 \leqslant j \leqslant k}\left(\frac{d_{i j}}{\sum_{1 \leqslant \ell \leqslant k} d_{i \ell}}+\frac{d_{i j}}{\sum_{1 \leqslant \ell \leqslant k} d_{\ell j}}\right)\left|I_{t}^{j}\right| .
$$

Set $X_{t}=\left(\left|I_{t}^{i}\right|\right)_{i \in[k]}$ and $A=\left(A_{i j}\right)_{1 \leqslant i, j \leqslant k}$, the matrix with entries

$$
A_{i j}=\frac{d_{i j}}{\sum_{1 \leqslant \ell \leqslant k} d_{i \ell}}+\frac{d_{i j}}{\sum_{1 \leqslant \ell \leqslant k} d_{\ell j}} \quad \text { for } 1 \leqslant i \neq j \leqslant k
$$

and $A_{i i}=1$ for $1 \leqslant i \leqslant k$. With this notation we obtain the recursive relation

$$
\mathbb{E}_{t}\left[X_{t+1}\right] \approx A \cdot X_{t},
$$

that is, we may expect that $X_{t} \approx \mathbb{E}_{t}\left[X_{t}\right] \approx A^{t} X_{0}$. If we then let $\lambda_{\max }$ denote the greatest eigenvalue of $A$, then we obtain that to leading order

$$
\left|I_{t}\right| \approx \lambda_{\max }^{t}
$$

Our aim is to show that pushepull is (at least) as fast as on the complete graph, that is, $\left|I_{t}\right| \succsim 3^{t}$, so we take a closer look at the eigenvalues of $A$. By construction $A$ is symmetric, so the largest eigenvalue equals $\sup _{\|x\|=1}\left\|x^{T} A x\right\|$, and the simple choice $x=k^{-1 / 2} \mathbf{1}$ yields

$$
\lambda_{\max } \geqslant \frac{\sum_{(i, j)} A_{i, j}}{k}=\frac{\sum_{j=1}^{k} 1+\sum_{i=1}^{k} \sum_{j=1}^{k} d_{i j} /\left(\sum_{\ell=1}^{k} d_{i \ell}\right)+\sum_{j=1}^{k} \sum_{i=1}^{k} d_{i j} /\left(\sum_{\ell=1}^{k} d_{\ell j}\right)}{k}=3 .
$$

This neat property leads us to the expected result

$$
T_{p p}(G)=(1+o(1)) \log _{\lambda_{\max }} n \leqslant(1+o(1)) \log _{3} n,
$$

and it also completes the informal argument that justifies the claim made in Theorems 1.3(b) and 1.5(b). In the rest of this section we will turn this argument step by step into a formal proof by filling in all missing pieces.

Obtaining an appropriate regular partition. An important ingredient in the previous sketch was the assumption that the given graph has a partition into a bounded number of equal parts, such that the bipartite graph induced by any two different parts looks like a random regular graph. This assumption is quite strong and very much not true in general. However, restricting ourselves to dense graphs we can actually come quite close to that. Let us begin with some definitions; the statements are taken from [30]. 
Definition 3.1 (density). Given a graph $G=(V, E)$ and two disjoint non-empty sets of vertices $X, Y \subseteq V$, we define the density of the pair $(X, Y)$ as

$$
d_{G}(X, Y)=\frac{e_{G}(X, Y)}{|X||Y|} .
$$

As usual, if the graph is clear from the context the index will be omitted. The next definition gives a partition that is close to the previously described properties; all sets in the partition have nearly the same size and nearly all pairs behave in a well-defined sense like regular bipartite random graphs.

Definition $3.2\left(\left(\varepsilon, k_{0}, K_{0}\right)\right.$-Szemerédi partition). Let $G=(V, E)$ and $k \in \mathbb{N}$. We call $\Pi=\left\{V_{i}\right\}_{i \in[k]}$ an $\left(\varepsilon, k_{0}, K_{0}\right)$-Szemerédi partition of $G$ if the following conditions are fulfilled.

(a) $V_{1} \dot{\cup} \ldots \dot{\cup} V_{k}=V$.

(b) $k_{0} \leqslant k \leqslant K_{0}$.

(c) $\left|V_{1}\right| \leqslant \cdots \leqslant\left|V_{k}\right| \leqslant\left|V_{1}\right|+1$.

(d) For all but at most $\varepsilon k^{2}$ pairs $\left(V_{i}, V_{j}\right)$ of $\Pi$ with $i<j$, we have that for all subsets $U_{i} \subseteq V_{i}$ and $U_{j} \subseteq V_{j}$ with $\left|U_{i}\right| \geqslant \varepsilon\left|V_{i}\right|$ and $\left|U_{j}\right| \geqslant \varepsilon\left|V_{j}\right|$,

$$
\left|d\left(U_{i}, U_{j}\right)-d\left(V_{i}, V_{j}\right)\right| \leqslant \varepsilon .
$$

A pair $\left(V_{i}, V_{j}\right)$ satisfying the last condition is called $\varepsilon$-regular. For pairs $\left(V_{i}, V_{j}\right)$ in $\Pi$ we will abbreviate $d\left(V_{i}, V_{j}\right)$ to $d_{i j}$.

Next we state Szemerédi's Regularity Lemma. It guarantees that we will have a Szemerédi partition if the underlying graph is large enough.

Lemma 3.10 ([30], The Regularity Lemma). For every $\varepsilon>0$ and every $k_{0} \in \mathbb{N}$ there exist $K_{0}=$ $K_{0}\left(\varepsilon, k_{0}\right)$ and $n_{0}$ such that every graph $G=(V, E)$ with at least $|V|=n \geqslant n_{0}$ vertices admits an $\left(\varepsilon, k_{0}, K_{0}\right)$-Szemerédi partition.

The next lemma gives a useful property of regular pairs. In particular, with the exception of a small set only, all other vertices have a degree that is close to $d N$, where $d$ is the density of the pair and $N$ is the number of vertices in each part. In fact the statement also is true for arbitrary but not too small subsets of the parts.

Lemma 3.11. Let $G=(V, E)$ be a graph, $\varepsilon>0$ and $U, U^{\prime} \subseteq V$. Suppose that $\left(U, U^{\prime}\right)$ is an $\varepsilon$ regular pair, and let $W \subseteq U^{\prime},|W| \geqslant \varepsilon\left|U^{\prime}\right|$. Furthermore, let $\mathcal{E}(U, W) \subseteq U$ be the largest set such that $\left|d(u, W)-d\left(U, U^{\prime}\right)\right| \geqslant \varepsilon$ for all $u \in \mathcal{E}(U, W)$. Then $|\mathcal{E}(U, W)| \leqslant 2 \varepsilon|U|$.

Proof. We will prove this by contradiction. Assume that $|\mathcal{E}(U, W)| \geqslant 2 \varepsilon|U|$. Let us write $\mathcal{E}(U, W)=S \cup L$, where

$$
S=\left\{u \in \mathcal{E}(U, W): d(u, W)<d\left(U, U^{\prime}\right)-\varepsilon\right\}, \quad L=\left\{u \in \mathcal{E}(U, W): d(u, W)>d\left(U, U^{\prime}\right)+\varepsilon\right\} .
$$

Then $|S| \geqslant \varepsilon|U|$ or $|L| \geqslant \varepsilon|U|$. In the former case

$$
d(S, W)=\frac{\sum_{u \in S} e(u, W)}{|S||W|}=\frac{\sum_{u \in S} d(u, W)}{|S|}<d\left(U, U^{\prime}\right)-\varepsilon .
$$

As $|S| \geqslant \varepsilon|U|,|W| \geqslant \varepsilon\left|U^{\prime}\right|$, this contradicts the assumption that $\left(U, U^{\prime}\right)$ is an $\varepsilon$-regular pair. The case $|L| \geqslant \varepsilon|U|$ follows analogously by showing that $d(L, W)>d\left(U, U^{\prime}\right)+\varepsilon$. 
We call the set $\mathcal{E}(U, W)$ in Lemma 3.11 the exceptional set of $U$ with respect to $W$. In particular, Lemma 3.11 implies that for every $\varepsilon$-regular pair $\left(U, U^{\prime}\right)$ and all $W \subseteq U^{\prime},|W| \geqslant(1-c \varepsilon)\left|U^{\prime}\right|, c>0$ we have

$$
\begin{aligned}
\left|d(u, W)-d\left(U, U^{\prime}\right)\right| & \leqslant\left|d(u, W)-d\left(u, U^{\prime}\right)\right|+\left|d\left(u, U^{\prime}\right)-d\left(U, U^{\prime}\right)\right| \\
& \leqslant(c+1) \varepsilon \text { for all } u \in U \backslash \mathcal{E}\left(U, U^{\prime}\right) .
\end{aligned}
$$

Having done these preparations we can now determine a partition that comes close to the initially described properties.

Lemma 3.12. Let $G_{n}=(V, E)$ be a graph on $n$ vertices such that $\delta_{G_{n}} \geqslant \alpha n$ for some $\alpha>0$. Then for all $\eta>0$ and $k_{0}>1 / \sqrt{\eta}$ there exists $n_{0}, K_{0} \in \mathbb{N}$ such that for all $G_{n}$ with $n \geqslant n_{0}$ there is an $\left(\eta, k_{0}, K_{0}\right)$-Szemerédi partition $\Pi=\left\{V_{i}\right\}_{i \in[k]}$ of $G_{n}$ with the following property. There is $F \subseteq \Pi$ with $|F| \leqslant \eta k$ such that, for all $V_{i} \in \Pi \backslash F$,

- there are at most $\eta k$ non- $\eta$-regular pairs $\left(V_{i}, V_{j}\right), j \in[k]$, and

- there exists an exceptional set $N_{i},\left|N_{i}\right| \leqslant \eta\left|V_{i}\right|$ such that

$$
d(u) \leqslant(1+\eta) \frac{n}{k} \sum_{1 \leqslant j \leqslant k} d\left(V_{i}, V_{j}\right) \quad \text { for all } u \in V_{i} \backslash N_{i} .
$$

Proof. According to Lemma 3.10, for all $\xi>0$ and $k_{0}>1 / \sqrt{\xi}$, there are $n_{0}, K_{0} \in \mathbb{N}$ such that for all $G_{n}$ with $n \geqslant n_{0}$ there is a $k \in \mathbb{N}$ and a $\left(\xi, k_{0}, K_{0}\right)$-Szemerédi partition $\Pi=\left\{V_{i}\right\}_{i \in[k]}$ of $G_{n}$. Let $F \subseteq \Pi$ contain the parts $V_{i} \in \Pi$ such that there are at least $\sqrt{\xi} k$ other parts $V_{j} \in \Pi$ such that the $\operatorname{pair}\left(V_{i}, V_{j}\right)$ is not $\xi$-regular. As there are at most $\xi k^{2}$ non- $\xi$-regular pairs, we infer that $|F| \leqslant \sqrt{\xi} k$. Let $V_{i} \in \Pi \backslash F$. Further, let $A_{i} \subseteq \Pi$ be such that $\left(V_{i}, V_{j}\right)$ is a $\xi$-regular pair for all $V_{j} \in \Pi \backslash A_{i}$ and $\left(V_{i}, V_{j}\right)$ is not $\xi$-regular for all $V_{j} \in A_{i}$. The definition of $F$ implies that $\left|A_{i}\right| \leqslant \sqrt{\xi k}$. For these $V_{j} \in \Pi \backslash A_{i}$ let $\mathcal{E}_{i}\left(V_{j}\right)=\mathcal{E}\left(V_{i}, V_{j}\right)$ be the exceptional set of $V_{i}$ with respect to $V_{j}$. On top of that let $N_{i} \subseteq V_{i}$ be the set of points in $V_{i}$ that are in at least $\sqrt{\xi} k$ exceptional sets with respect to parts in $\Pi \backslash A_{i}$. As there are at most $k$ exceptional sets and by Lemma 3.11 each exceptional set has at most $2 \xi\left|V_{i}\right|$ vertices, we get that $\left|N_{i}\right| \leqslant 2 \sqrt{\xi}\left|V_{i}\right|$. Let $V_{i} \in \Pi \backslash F, u \in V_{i} \backslash N_{i}$ and let $B(u) \subseteq \Pi \backslash A_{i}$ be the set of parts such that $u \in \mathcal{E}_{i}\left(V_{j}\right)$ for all $V_{j} \in B$. Then $|B| \leqslant \sqrt{\xi} k$ and

$$
\begin{aligned}
d(u) & =\sum_{1 \leqslant j \leqslant k}\left|V_{j}\right| d\left(u, V_{j}\right) \\
& =\left(\sum_{V_{j} \in A_{i} \cup B}\left|V_{j}\right| d\left(u, V_{j}\right)+\sum_{V_{j} \in \Pi \backslash\left(A_{i} \cup B\right)}\left|V_{j}\right| d\left(u, V_{j}\right)\right) \\
& \leqslant\left|N(u) \cap\left(\bigcup_{V_{j} \in A_{i} \cup B \cup\left\{V_{i}\right\}} V_{j}\right)\right|+\sum_{1 \leqslant j \leqslant k}\left|V_{j}\right|\left(d\left(V_{i}, V_{j}\right)+\xi\right) .
\end{aligned}
$$

By the definition of $F$ and as $u \in V_{i} \backslash N_{i}$, we get that

$$
\left|\bigcup_{V_{j} \in A_{i} \cup B \cup\left\{V_{i}\right\}} V_{j}\right| \leqslant(\sqrt{\xi} k+\sqrt{\xi} k+1)(n / k+1) \leqslant 3 \sqrt{\xi} n .
$$

With that at hand and by using $d(u) \geqslant \alpha n$ and the fact that the sizes of the parts in $\Pi$ differ by at most one, we obtain

$$
d(u) \leqslant 3 \sqrt{\xi} n+\frac{n}{k} \sum_{1 \leqslant j \leqslant k} d\left(V_{i}, V_{j}\right)+2 \xi n \leqslant \frac{n}{k} \sum_{1 \leqslant j \leqslant k} d\left(V_{i}, V_{j}\right)+5 \sqrt{\xi} d(u) / \alpha .
$$


Let $\eta>0$. Choosing $\xi$ small enough such that

$$
\max \{\xi, 2 \sqrt{\xi}, 1 /(1-5 \sqrt{\xi} / \alpha)-1\} \leqslant \eta
$$

implies the claim.

The recursion relation. In this section we exploit the properties of the partition to study the expected number of informed vertices after one additional round; our aim is to establish a precise version of (3.16). In the remainder let $\|A\|_{F}=\left(\sum_{1 \leqslant i \leqslant n} \sum_{1 \leqslant j \leqslant n}\left|a_{i, j}\right|^{2}\right)^{1 / 2}$ denote the Frobenius norm of a matrix $A \in \mathbb{R}^{n \times n}$.

For the next lemma consider the setting of Theorems 1.3(b) and 1.5(b), that is, we are given an expander sequence $\left(G_{n}\right)_{n \in \mathbb{N}}$ with minimal degree $\delta_{n} \geqslant \alpha n$ for some $\alpha>0$ and an $\varepsilon>0$. We obtain a sequence of graphs $\left(\tilde{G}_{n}\right)_{n \in \mathbb{N}}$ by deleting up to a $1 / 2-\varepsilon$ fraction of the edges at each vertex in $G_{n}$. Further, let $\eta>0, k_{0} \in \mathbb{N}$ and $\Pi=\left\{V_{i}\right\}_{i \in[k]}$ be the $\left(\eta, k_{0}, K_{0}\right)$-Szemerédi partition of $\tilde{G}_{n}$ as given by Lemma 3.12. For that partition define $\mathcal{E}_{i, j}:=\mathcal{E}\left(V_{i}, V_{j}\right)$ as the exceptional set of $V_{i}$ with respect to $V_{j}$ given by Lemma 3.11, $i \neq j \in[k], F$ and $N_{i}$ as the exceptional sets from Lemma 3.12, $i \in \Pi \backslash F$. Moreover, let $\Pi_{i}=\left\{V_{j} \in \Pi \backslash F:\left(V_{i}, V_{j}\right)\right.$ is $\eta$-regular $\}$ and note that

$$
\left|\Pi_{i}\right| \geqslant(1-2 \eta) k, \quad\left|N_{i}\right| \leqslant \eta\left|V_{i}\right| \quad \text { and } \quad\left|\mathcal{E}_{i, j}\right| \leqslant 2 \eta\left|V_{i}\right| \quad \text { for all } i \in \Pi \backslash F, j \in \Pi_{i} .
$$

Finally, define

$$
\mathcal{H}_{i, j^{\prime}}=N_{i} \cup \mathcal{E}_{i, j^{\prime}}, \quad i \in \Pi \backslash F \text { and } j \in \Pi_{i}
$$

and

$$
X_{t, i, j}=\left|I_{t}^{(p p)} \cap\left(V_{i} \backslash\left(N_{i} \cup \mathcal{E}_{i, j}\right)\right)\right|, \quad i \in \Pi \backslash F \text { and } j \in \Pi_{i}
$$

as well as

$$
X_{t, i}=\min _{j \in \Pi_{i}} X_{t, i, j}, \quad i \in \Pi \backslash F .
$$

This definition guarantees that $\left|I_{t}^{(p p)}\right| \geqslant\left\|X_{t}\right\|_{1}$. The cornerstone of our proof is the following lemma, which bounds the growth of $X_{t}=\left(X_{t, i}\right)_{i \in \Pi \backslash F}$ after one round.

Lemma 3.13. Consider the situation as described above and assume additionally that $\left|X_{t, i}\right| \geqslant$ $\log \log n$ for all $i \in \Pi \backslash F$ and that $\left|I_{t}^{(p p)}\right| \leqslant n / \log n$. Then, for all $v>0$ and $n$ large enough, there exists a symmetric matrix $A$ with biggest eigenvalue $\lambda_{\max } \geqslant 1+2 q-v$ and an error matrix $\Delta A$ with $\|\Delta A\|_{F} \leqslant v$ such that w.h.p.

$$
X_{t+1} \geqslant(A+\Delta A) X_{t}
$$

Proof. We set $I_{t}^{\mathcal{P}, i}=I_{t}^{\mathcal{P}} \cap V_{i}, U_{t}^{\mathcal{P}, i}=U_{t}^{\mathcal{P}} \cap V_{i}$ for $\mathcal{P} \in\{p u s h, p u l l, p p\}$ and let

$$
I_{t+1}^{\mathcal{P}, i, j} \backslash I_{t}=\left\{u \in U_{t} \cap V_{i} \mid \text { there is } v \in I_{t} \cap V_{j} \text { such that } u \text { gets informed by } v \text { using } \mathcal{P}\right\}
$$

be the vertices in $V_{i}$ newly informed in round $t+1$ by operations involving only vertices from $V_{i}$ and $V_{j}$. Let $(i, j) \in \Pi \backslash F$. For all $u \in U_{t}^{i}$ we know that $d(u) \geqslant \alpha n / 2$. Moreover, $\left|I_{t}^{i}\right| \leqslant\left|I_{t}\right| \leqslant n / \log n$. Thus the probability of $u \in U_{t}^{i}$ being informed by vertices in $I_{t}^{j}$ via pull is $q\left|N(u) \cap I_{t}^{j}\right| /|N(u)|=$ $o(1)$. As the events of $u$ being informed by push and pull are independent,

$$
\mathbb{P}\left[u \in I_{t+1}^{(\text {push }), i, j} \cap I_{t+1}^{(\text {pull }), i, j}\right]=o(1) \mathbb{P}\left[u \in I_{t+1}^{(\text {push }), i, j}\right] .
$$

Thus, for any set $S \in V$,

$$
\mathbb{E}\left[\left|\left(I_{t+1}^{(p p), i, j} \backslash I_{t}\right) \cap S\right|\right]=(1-o(1))\left(\mathbb{E}\left[\left|\left(I_{t+1}^{(\text {pull }), i, j} \backslash I_{t}\right) \cap S\right|\right]+\mathbb{E}\left[\left|\left(I_{t+1}^{(\text {push }), i, j} \backslash I_{t}\right) \cap S\right|\right]\right) .
$$


Let $i \in \Pi \backslash F$ and $j \in \Pi_{i}$. We start by determining the expected number of vertices informed by pull. Further, set

$$
D_{i}=(1+\eta) \frac{n}{k} \sum_{1 \leqslant \ell \leqslant k} d_{i \ell}
$$

According to Lemma 3.12, all $v \in U_{t}^{i} \backslash N_{i}$ have degree less than $D_{i}$. Let $j^{\prime} \in \Pi_{i}$. Then

$$
\mathbb{E}_{t}\left[\left|I_{t+1}^{(p u l l), i, j} \backslash\left(I_{t} \cup \mathcal{H}_{i, j^{\prime}}\right)\right|\right]=\sum_{u \in U_{t}^{i} \backslash \mathcal{H}_{i, j^{\prime}}} q \frac{\left|N(u) \cap I_{t}^{j}\right|}{|N(u)|} \geqslant q \frac{e\left(U_{t}^{i} \backslash \mathcal{H}_{i, j^{\prime}}, I_{t}^{j}\right)}{D_{i}} .
$$

Since $\left|I_{t}^{i}\right| \leqslant\left|I_{t}\right| \leqslant n / \log n$, we get with room to spare that $\left|U_{t}^{i} \backslash \mathcal{H}_{i, j^{\prime}}\right| \geqslant(1-5 \eta) n / k$ for $n$ large enough and all $j^{\prime} \in \Pi_{i}$. Applying (3.17), where we choose $W=U_{t}^{i} \backslash \mathcal{H}_{i, j^{\prime}}$, yields $\mid d\left(U_{t}^{i} \backslash \mathcal{H}_{i, j^{\prime}}, u\right)-$ $d_{i j} \mid \leqslant 6 \eta$ for all $u \in V_{j} \backslash \mathcal{E}_{j, i}$. Thus

$$
\begin{aligned}
\mathbb{E}_{t}\left[\left|I_{t+1}^{(p u l l), i, j} \backslash\left(I_{t} \cup \mathcal{H}_{i, j^{\prime}}\right)\right|\right] & \geqslant q \frac{\left(d_{i j}-6 \eta\right)\left|U_{t}^{i} \backslash \mathcal{H}_{i, j^{\prime}}\right|\left|I_{t}^{j} \backslash \mathcal{E}_{j, i}\right|}{D_{i}} \\
& \geqslant(1-5 \eta) q \frac{\left(d_{i j}-6 \eta\right)\left|I_{t}^{j} \backslash\left(\mathcal{E}_{j, i} \cup N_{j}\right)\right|}{D_{i} k / n} .
\end{aligned}
$$

As $D_{i}=(1+\eta) n / k \sum_{1 \leqslant \ell \leqslant k} d_{i \ell}$, we get for

$$
c_{1}:=(1-6 \eta)(1+\eta)^{-1}
$$

with $X_{t, j, i}=\left|I_{t}^{j} \backslash\left(\mathcal{E}_{j, i} \cup N_{j}\right)\right|$ that

$$
\mathbb{E}_{t}\left[\left|I_{t+1}^{(p u l l), i, j} \backslash\left(I_{t} \cup \mathcal{H}_{i, j^{\prime}}\right)\right|\right] \geqslant c_{1} \cdot q \frac{\left(d_{i j}-6 \eta\right) X_{t, j, i}}{\sum_{1 \leqslant \ell \leqslant k} d_{i \ell}} \quad \text { for all } i \in \Pi \backslash F \text { and } j, j^{\prime} \in \Pi_{i} .
$$

We continue with push. Let $i \in \Pi \backslash F$ and $j, j^{\prime} \in \Pi_{i}$, and set (as before)

$$
D_{j}=(1+\eta) \frac{n}{k} \sum_{1 \leqslant \ell \leqslant k} d_{\ell j}
$$

Then

$$
\mathbb{E}_{t}\left[\left|I_{t+1}^{(p u s h), i, j} \backslash\left(I_{t} \cup \mathcal{H}_{i, j^{\prime}}\right)\right|\right]=\sum_{u \in U_{t}^{i} \backslash \mathcal{H}_{i, j^{\prime}}}\left(1-\prod_{v \in N(u) \cap I_{t}^{j}}\left(1-\frac{q}{|N(v)|}\right)\right) .
$$

According to Lemma 3.12 all $v \in I_{t}^{j} \backslash N_{j}$ have degree less than $D_{j}$ and furthermore $\left|I_{t}\right|=o(n)=$ $o\left(D_{j}\right)$. Thus (3.3) yields the estimate

$$
\begin{aligned}
\mathbb{E}_{t}\left[\left|I_{t+1}^{(p u s h), i, j} \backslash\left(I_{t} \cup \mathcal{H}_{i, j^{\prime}}\right)\right|\right] & \geqslant \sum_{u \in U_{t}^{i} \backslash \mathcal{H}_{i, j^{\prime}}}\left(1-\left(1-\frac{q}{D_{j}}\right)^{\left|N(u) \cap\left(I_{t}^{j} \backslash N_{j}\right)\right|}\right) \\
& \geqslant(1-o(1)) \sum_{u \in U_{t}^{i} \backslash \mathcal{H}_{i, j^{\prime}}} q \frac{\left|N(u) \cap\left(I_{t}^{j} \backslash N_{j}\right)\right|}{D_{j}} .
\end{aligned}
$$

The remaining steps are similar to the previously considered case of pull. By assumption we have that $\left|I_{t}^{j} \backslash \mathcal{H}_{j, i}\right|=X_{t, j, i}$ and as $\left|I_{t}^{i}\right| \leqslant\left|I_{t}\right| \leqslant n / \log n$ we obtain that $\left|U_{t}^{i} \backslash \mathcal{H}_{i, j^{\prime}}\right| \geqslant(1-5 \eta) n / k$ for $n$ large 
enough and all $j^{\prime} \in \Pi_{i}$. Using (3.17) we obtain that $\left|d\left(U_{t}^{i} \backslash \mathcal{H}_{i, j^{\prime}}, u\right)-d_{i j}\right| \leqslant 6 \eta$ for all $u \in V_{j} \backslash \mathcal{E}_{j, i}$. Thus

$$
\begin{aligned}
\mathbb{E}_{t}\left[\left|I_{t+1}^{(p u s h), i, j} \backslash\left(I_{t} \cup \mathcal{H}_{i, j^{\prime}}\right)\right|\right] & \geqslant(q-o(1)) \frac{e\left(U_{t}^{i} \backslash \mathcal{H}_{i, j^{\prime}}, I_{t}^{j} \backslash\left(N_{j} \cup \mathcal{E}_{j, i}\right)\right)}{D_{j}} \\
& \geqslant(q-o(1)) \frac{\left(d_{i j}-6 \eta\right)\left|U_{t}^{i} \backslash \mathcal{H}_{i, j^{\prime}}\right| X_{t, j, i}}{D_{j}} .
\end{aligned}
$$

Using the fact that $D_{j}=(1+\eta) n / k \sum_{1 \leqslant \ell \leqslant k} d_{\ell j}$, we get for the same constant $c_{1}$ as in (3.20) and $n$ large enough

$$
\mathbb{E}_{t}\left[\left|I_{t+1}^{(p u s h), i, j} \backslash\left(I_{t} \cup \mathcal{H}_{i, j^{\prime}}\right)\right|\right] \geqslant c_{1} \cdot q \frac{\left(d_{i j}-6 \eta\right) X_{t, j, i}}{\sum_{1 \leqslant \ell \leqslant k} d_{\ell j}} \quad \text { for all } i \in \Pi \backslash F \text { and } j, j^{\prime} \in \Pi_{i} .
$$

With (3.19), we can combine the results for pull, (3.20), and push, (3.22), to get for $c_{2}:=c_{1}-\eta$

$$
\mathbb{E}_{t}\left[\left|I_{t+1}^{(p p), i, j} \backslash\left(I_{t}^{i} \cup \mathcal{H}_{i, j^{\prime}}\right)\right|\right] \geqslant c_{2} \cdot q\left(\frac{d_{i j}-6 \eta}{\sum_{1 \leqslant \ell \leqslant k} d_{i \ell}}+\frac{d_{i j}-6 \eta}{\sum_{1 \leqslant \ell \leqslant k} d_{\ell j}}\right) X_{t, j, i} \quad \text { for all } i \in \Pi \backslash F, j, j^{\prime} \in \Pi_{i} .
$$

Next we will show how we can exploit (3.23) to obtain (a lower bound for) $\mathbb{E}_{t}\left[\left|\left(I_{t+1}^{(p p), i} \backslash I_{t}\right)\right|\right]$. Let $i \in \Pi \backslash F$ and $u \in U_{t}^{i}$. Using $\left|I_{t}\right|=o(n)$ and (3.3) we obtain

$$
\mathbb{P}_{t}\left[u \in I_{t+1}^{(\text {push }), i} \backslash I_{t}\right]=1-\prod_{i \in N(u) \cap I_{t}}\left(1-\frac{1}{|N(i)|}\right)=(1-o(1)) \sum_{i \in N(u) \cap I_{t}} \frac{1}{|N(i)|} .
$$

Let $W \subseteq V$. Using (3.19), the previous equation and that $\Pi$ is a partition, we get

$$
\begin{aligned}
\mathbb{E}_{t}\left[\left|\left(I_{t+1}^{(p p), i} \backslash I_{t}\right) \cap W\right|\right] & =(1-o(1)) \sum_{u \in U_{t}^{i} \cap W}\left(\frac{\left|N(u) \cap I_{t}\right|}{|N(u)|}+\sum_{i \in N(u) \cap I_{t}} \frac{1}{|N(i)|}\right) \\
& =(1-o(1)) \sum_{u \in U_{t}^{i} \cap W}\left(\sum_{j \in[k]}\left(\frac{\left|N(u) \cap I_{t} \cap V_{j}\right|}{|N(u)|}+\sum_{i \in N(u) \cap I_{t} \cap V_{j}} \frac{1}{|N(i)|}\right)\right) \\
& =(1-o(1)) \sum_{j \in[k]} \mathbb{E}_{t}\left[\left|\left(I_{t+1}^{(p p), i, j} \backslash I_{t}\right) \cap W\right|\right] .
\end{aligned}
$$

Choose $W=V \backslash \mathcal{H}_{i, j^{\prime}}$. Then the previous equation implies

$$
\mathbb{E}_{t}\left[\left|I_{t+1}^{(p p), i} \backslash\left(I_{t} \cup \mathcal{H}_{i, j^{\prime}}\right)\right|\right] \geqslant(1-o(1)) \sum_{j \in \Pi \backslash F} \mathbb{E}_{t}\left[\left|I_{t+1}^{(p p), i, j} \backslash\left(I_{t} \cup \mathcal{H}_{i, j^{\prime}}\right)\right|\right] \quad \text { for all } i \in \Pi \backslash F, j^{\prime} \in \Pi_{i},
$$

which in turn, using (3.23) and $X_{t, j, i} \geqslant X_{t, j}$ for all $j \in \Pi \backslash F$ and $i \in \Pi_{j}$, implies for $c:=c_{2}-\eta$

$$
\mathbb{E}_{t}\left[X_{t+1, i, j^{\prime}}\right] \geqslant X_{t, i}+c \cdot q \sum_{j \in \Pi_{i}}\left(\frac{d_{i j}-6 \eta}{\sum_{1 \leqslant \ell \leqslant k} d_{i \ell}}+\frac{d_{i j}-6 \eta}{\sum_{1 \leqslant \ell \leqslant k} d_{\ell j}}\right) X_{t, j} \quad \text { for all } i \in \Pi \backslash F, j^{\prime} \in \Pi_{i} .
$$

Assume that (3.24) holds not only in expectation but also for a slightly smaller $c$, say $c-\eta$, with high probability. We are going to show this at the end of the proof. Using this assumption and a union bound over $j^{\prime} \in \Pi_{i}$ gives w.h.p.

$$
X_{t+1, i}=\min _{j^{\prime} \in \Pi_{i}} X_{t+1, i, j^{\prime}} \geqslant\left\langle a_{i},\left(X_{t, j}\right)_{j \in \Pi_{i}}\right\rangle \quad \text { for all } i \in \Pi \backslash F,
$$


where for $i \in \Pi \backslash F$ and $j \in \Pi_{i}$ we have

$$
a_{i j}=\mathbb{1}[i=j]+c \cdot q\left(\frac{d_{i j}-6 \eta}{\sum_{1 \leqslant \ell \leqslant k} d_{i \ell}}+\frac{d_{i j}-6 \eta}{\sum_{1 \leqslant \ell \leqslant k} d_{\ell j}}\right) .
$$

Let $A$ be the $|\Pi \backslash F| \times|\Pi \backslash F|$ matrix with entries as in the previous equation, that is, $A=$ $\left(a_{i j}\right)_{(i, j) \in(\Pi \backslash F)^{2}}$ is given by (3.26) for all $(i, j) \in(\Pi \backslash F)^{2}$. Note that $A$ is symmetric. Then we obtain from (3.25)

$$
X_{t+1} \geqslant B \cdot X_{t},
$$

with $B=A+\Delta A$, where

$$
(\Delta A)_{i j}= \begin{cases}0 & i \in \Pi \backslash F \text { and } j \in \Pi_{i}, \\ -a_{i j} & i \in \Pi \backslash F \text { and } j \in \Pi \backslash\left(F \cup \Pi_{i}\right) .\end{cases}
$$

Set

$$
F^{\prime}:=\left\{(i, j) \in(\Pi \backslash F)^{2} \mid j \in \Pi \backslash\left(F \cup \Pi_{i}\right)\right\} .
$$

As $d(u) \geqslant \alpha n / 2$ for all $u \in V$ and some $\alpha>0$, we also know that $\sum_{1 \leqslant \ell \leqslant k} d_{\ell j} \geqslant k \alpha / 2$. Together with $0 \leqslant d_{i, j} \leqslant 1$ for all $(i, j) \in[k]^{2}$, we get that

$$
\left|\frac{d_{i j}-6 \eta}{\sum_{1 \leqslant \ell \leqslant k} d_{i \ell}}\right| \leqslant \frac{2}{\alpha k} .
$$

Using the fact that $\left|F^{\prime}\right| \leqslant 2 \eta k^{2}$ (see (3.18)), we obtain

$$
\|\Delta A\|_{F}^{2}=\sum_{(i, j) \in F^{\prime}} a_{i j}^{2} \leqslant \sum_{(i, j) \in F^{\prime}}\left(\frac{4}{\alpha k}\right)^{2} \leqslant 2 \eta k^{2}\left(\frac{4}{\alpha k}\right)^{2}=\frac{4^{2} \cdot 2 \cdot \eta}{\alpha^{2}}
$$

and thus $\|\Delta A\|_{F} \leqslant 4 \sqrt{2 \eta} / \alpha$. This leaves us with bounding the biggest eigenvalue $\lambda_{\max }$ of $A$. Using the well-known inequality for symmetric matrices,

$$
\lambda_{\max } \geqslant \sum_{(i, j) \in(\Pi \backslash F)^{2}} A_{i j} /|\Pi \backslash F|,
$$

we obtain

$$
\begin{aligned}
\lambda_{\max } \geqslant & \frac{1}{|\Pi \backslash F|} \sum_{(i, j) \in(\Pi \backslash F)^{2}} a_{i j} \\
\geqslant & \frac{1}{|\Pi \backslash F|}\left(\sum_{(i, i) \in(\Pi \backslash F)^{2}} 1+\sum_{(i, j) \in[k]^{2}} \frac{c q\left(d_{i j}-6 \eta\right)}{\sum_{1 \leqslant \ell \leqslant k} d_{i \ell}}\right. \\
& \left.+\sum_{(i, j) \in[k]^{2}} \frac{c q\left(d_{i j}-6 \eta\right)}{\sum_{1 \leqslant \ell \leqslant k} d_{\ell j}}-2 \sum_{i \in[k] \backslash(\Pi \backslash F)} \sum_{j \in[k]} \frac{c q}{\sum_{1 \leqslant \ell \leqslant k} d_{\ell j}}\right) .
\end{aligned}
$$

Note that $|\Pi \backslash F| \geqslant(1-\eta) k, \quad|[k] \backslash(\Pi \backslash F)| \leqslant \eta k$. Moreover, $\quad \sum_{1 \leqslant \ell \leqslant k} d_{\ell j} \geqslant \alpha k / 2$ for all $j \in[k]$. Thus

$$
\lambda_{\max } \geqslant 1+\frac{1}{k}\left(c q k+c q k-12 c q \sum_{(i, j) \in[k]^{2}} \frac{\eta}{\sum_{1 \leqslant \ell \leqslant k} d_{\ell j}}-2 c q \frac{\eta k^{2}}{\alpha k / 2}\right) \geqslant 1+2 c q(1-8 \eta / \alpha) .
$$

Choosing $\eta$ small enough such that $2 q(1-c(1-8 \eta / \alpha)), 4 \sqrt{2 \eta} / \alpha \leqslant v$ implies the claim of this lemma. 
This leaves us with proving that (3.24) also holds with high probability. As $\left|I_{t+1}^{(p p)}\right|$ conditioned on $I_{t}$ is a self-bounding function, so is $\left|I_{t+1}^{(p p), i} \backslash I_{t}^{i}\right|$ for all $i \in \Pi \backslash F$ and therefore also $\mid I_{t+1}^{(p p), i} \backslash\left(I_{t} \cup\right.$ $\left.\mathcal{H}_{i, j^{\prime}}\right) \mid=: Y_{t+1, i, j^{\prime}}$ for all $i \in \Pi \backslash F$ and $j^{\prime} \in \Pi_{i}$. Note that $Y_{t+1, i, j^{\prime}}=X_{t+1, i, j^{\prime}}-X_{t, i, j^{\prime}}$. Lemma 2.2 yields that

$$
\mathbb{P}_{t}\left[Y_{t+1, i, j^{\prime}} \geqslant\left(1-\mathbb{E}_{t}\left[Y_{t+1, i, j^{\prime}}\right]^{-1 / 3}\right) \mathbb{E}_{t}\left[Y_{t+1, i, j^{\prime}}\right]\right] \geqslant 1-\mathbb{E}_{t}\left[Y_{t+1, i, j^{\prime}}\right]^{-1 / 3}
$$

and therefore, setting

$$
Z_{t, i}=c \cdot q \sum_{j \in \Pi_{i}}\left(\frac{d_{i j}-6 \eta}{\sum_{1 \leqslant \ell \leqslant k} d_{i \ell}}+\frac{d_{i j}-6 \eta}{\sum_{1 \leqslant \ell \leqslant k} d_{\ell j}}\right) X_{t, j} \quad \text { for all } i \in \Pi \backslash F,
$$

and using (3.24), i.e. $\mathbb{E}_{t}\left[Y_{t+1, i, j^{\prime}}\right] \geqslant Z_{t, i}$ for all $i \in \Pi \backslash F$ and $j^{\prime} \in \Pi_{i}$, we get with probability at least $1-k^{3} Z_{t, i}^{-1 / 3}$

$$
Y_{t+1, i, j^{\prime}} \geqslant\left(1-Z_{t, i}^{-1 / 3}\right) Z_{t, i} \quad \text { for all } i \in \Pi \backslash F \text { and } j^{\prime} \in \Pi_{i}
$$

This and $\left|I_{t}^{i}\right| \geqslant X_{t, i}$ for all $i \in \Pi \backslash F$ implies that (3.24) also holds with high probability for a marginally smaller $c$, as claimed.

Extension. We now solve the linear recurrence relation above and extend it to more than one round to get an upper bound on the runtime of pusherpull. We first state a Chernoff bound that will be very useful in the next lemma.

Lemma 3.14 ([26]). Let $\varepsilon, \delta>0$. Suppose that $X_{1}, \ldots, X_{n}$ are independent geometric random variables with parameter $\delta$, so $\mathbb{E}\left[X_{i}\right]=1 / \delta$ for each $i$. Let $X:=\sum_{1 \leqslant i \leqslant n} X_{i}, \mu=\mathbb{E}[X]=n / \delta$. Then

$$
\mathbb{P}[X \geqslant(1+\varepsilon) \mu] \leqslant e^{-n(\varepsilon-\log (1+\varepsilon))} \leqslant e^{-\varepsilon^{2} n / 2(1+\varepsilon)}
$$

Together with Lemma 2.11 the following lemma implies Theorems 1.3(b) and 1.5(b).

Lemma 3.15. Consider the setting of Theorems $1.3(b)$ and $1.5(b)$ and let $I_{t}=I_{t}^{(p p)}$. The following statements hold w.h.p.

(a) Let $S \subseteq V_{n},|S|=\Theta(n)$. Then there is $t=\Theta(\log \log n)$ such that w.h.p. $\left|I_{t}\right| \geqslant\left|I_{t} \cap S\right| \geqslant$ $\log \log n$.

(b) Let $\log \log n \leqslant\left|I_{t}\right| \leqslant n / \log n$. Then there is $\tau \leqslant \log _{1+2 q}\left(n /\left|I_{t}\right|\right)+o(\log n)$ such that $\left|I_{t+\tau}\right|>n / \log n$.

(c) Let $n / \log n \leqslant\left|I_{t}\right| \leqslant n-n / \log n$. Then there is $\tau=o(\log n)$ such that $\left|I_{t+\tau}\right|>n-n / \log n$.

(d) Let $\left|I_{t}\right| \geqslant n-n / \log n$ and $q=1$. Then there is $\tau=o(\log n)$ such that $\left|I_{t+\tau}\right|=n$.

Proof. As $\left|I_{t}^{(p p)}\right| \geqslant\left|I_{t}^{(p u l l)}\right|$ clearly (c) and (d) follow from Lemma 3.2. We show (a) by determining a lower bound for the probability that an arbitrary vertex gets informed after a constant number of rounds. Set $\beta=\min \{\alpha, \epsilon\}$, let $S_{0}=\{u\}$ and choose $w \in V, w \neq u$. By Lemma 2.9 there is $d \leqslant$ $8 / \beta^{2}+2$ and $c=\left(\beta^{4} / 64\right)^{8 / \beta^{2}+3} \in(0,1)$ such that there are at least $c n^{d-1}$ paths of (edge) length $d$ from $u$ to $w$. Let $\gamma=\left(u, v_{1}, \ldots, v_{d-1}, w\right)$ be such a path from $u$ to $w$, and let $A_{\gamma}$ denote the event that $w$ is informed via $\gamma$ after exactly $d$ rounds performing only push operations, that is, $A_{\gamma}$ is the event that in the first round the randomly selected neighbour of $u$ is $v_{1}$, in the second round the randomly selected neighbour of $v_{1}$ is $v_{2}$ and so forth, until in the $d$ th round the randomly selected neighbour of $v_{d-1}$ is $w$. Obviously, the probability of $A_{\gamma}$ is bounded from below by $n^{-d}$. Further, let $\gamma^{\prime} \neq \gamma$ be another path from $u$ to $w$ with length $d$. As $\gamma$ and $\gamma^{\prime}$ differ by at least one edge we 
readily obtain that $\mathbb{P}\left[A_{\gamma} \cap A_{\gamma^{\prime}}\right]=0$. Let $\Gamma$ denote the set of all paths with length $d$ from $u$ to $w$. Having done these preparations we use them to conclude for all $w \in V$ and $t \geqslant 0$

$$
\mathbb{P}_{t}\left[w \in I_{t+d}\right] \geqslant \mathbb{P}_{t}\left[\bigcup_{\gamma \in \Gamma} A_{\gamma}\right] \geqslant \sum_{\gamma \in \Gamma} \mathbb{P}_{t}\left[A_{\gamma}\right] \geqslant \sum_{\gamma \in \Gamma} n^{-d} \geqslant \frac{c}{n} .
$$

We define a modified protocol as follows. Wait $d:=\left\lceil 8 / \beta^{2}+2\right\rceil$ rounds, after that with probability $c$ choose one uninformed vertex uniformly at random and set it as informed. Repeat. Call the vertices informed by this algorithm $I_{t}^{\star}$. Then the probability of any vertex being informed after $d$ rounds is

$$
\mathbb{P}_{t}\left[v \in I_{t+d}^{\star} \mid v \notin I_{t}^{\star}\right]=c / n
$$

Thus, for any $t \geqslant 0$,

$$
\mathbb{P}_{t}\left[v \in I_{t+d} \mid v \in U_{t}\right] \geqslant \mathbb{P}_{t}\left[v \in I_{t+d}^{\star} \mid v \notin I_{t}^{\star}\right]=c / n .
$$

Note that for any $s \in \mathbb{N}$ the set $I_{s d}^{\star}$ is generated by a very simple procedure: $s$ times independently, with probability $c$, we choose a random vertex and put it into $I_{s d}^{\star}$. Thus $\left|I_{s d}^{\star} \cap S\right|$ is binomially distributed with $s$ trials, where each one has success probability $c|S| / n=\Theta(c)$; it follows readily that $\left|I_{s d}^{\star} \cap S\right|$ concentrates around a multiple of $s$ for large $s$, and the claim follows by choosing $s=\Theta(\log \log n)$.

This leaves (b) to be shown. Part (a) implies that there is some $t_{0}=o(\log n)$ such that $X_{t_{0}, i}=$ $\Theta(\log \log n)$ for all $i \in \Pi \backslash F$ by choosing $S=V_{i} \backslash\left(N_{i} \cup \mathcal{E}_{i, j}\right), j \in \Pi_{i}$ and applying a union bound over $i$ and $j$. Thus we can apply Lemma 3.13. It gives w.h.p., say with probability $1-g(n)=1-$ $o(1)$, that $X_{t+1} \geqslant(A+\Delta A) X_{t}$, $A$ has maximal eigenvalue $\lambda_{\max }(A) \geqslant 1+2 q-v$ and $\|\Delta A\|_{F} \leqslant v$. Then $B:=A+\triangle A$ has maximal eigenvalue

$$
\lambda_{\max }(B) \geqslant \lambda_{\max }(A)-\|\Delta A\|_{F} \geqslant 1+2 q-2 v
$$

(Theorem of Wielandt and Hoffmann; see e.g. [24]).

Set $f(n):=(\log (n / \log n))^{2 / 3}$. Our assumptions guarantee that $f(n)=\omega(1)$ and $f(n)=$ $o(\log n)$. Moreover, set

$$
\tau:=\frac{1}{1-g(n)} \cdot \frac{\log (n / \log n)}{\log \left(\lambda_{\max }(B)\right)}+f(n)=\frac{\log n}{\log \left(\lambda_{\max }(B)\right)}+o(\log n) .
$$

Let $\left(X_{i}\right)_{i \in \mathbb{N}}$ be independent and identically distributed geometric random variables with expectation $1-g(n)$. Set $X=X_{1}+X_{2}+\cdots+X_{\mathcal{T}}$ with $\mathcal{T}=\log (n / \log n) / \log \left(\lambda_{\max }(B)\right)$. We show that $\mathbb{P}[X \leqslant \tau]=1-o(1)$. To see this, note first that by linearity of expectation $\mathbb{E}[X]=\tau-f(n)$. Then, by Lemma 3.14,

$$
\mathbb{P}[X \leqslant \tau]=\mathbb{P}\left[X \leqslant\left(1+\frac{f(n)}{\tau+f(n)}\right) \mathbb{E}[X]\right] \geqslant 1-\exp \left(-\Theta\left(\frac{f(n)^{2}}{\tau}\right)\right)=1-o(1) .
$$

Thus we have w.h.p.

$$
\left|I_{t+\tau}\right| \geqslant\left\|X_{t+\tau}\right\|_{1} \geqslant\left\|B^{\mathcal{T}} X_{t_{0}}\right\|_{1} .
$$

Let $v$ be an eigenvector of $B$ to $\lambda_{\max }(B)$. As $v \neq 0$ there is an index $\ell$ such that $v_{\ell} \neq 0$. Without loss of generality we can assume that $v_{\ell}=1$, as $v / v_{\ell}$ is also an eigenvector to $\lambda_{\max }(B)$. Thus $\left(B^{\mathcal{T}} v\right)_{\ell}=$ $\lambda_{\max }(B)^{\mathcal{T}},\left(B^{\mathcal{T}}\left(X_{t_{0}}-v\right)\right)_{i} \geqslant 0$ for all $1 \leqslant i \leqslant k$ and therefore

$$
\left|I_{t+\tau}\right| \geqslant\left(B^{\mathcal{T}} X_{t_{0}}\right)_{\ell} \geqslant\left(B^{\mathcal{T}}\left(v+X_{t_{0}}-v\right)\right)_{\ell}=\left(B^{\mathcal{T}} v\right)_{\ell}+\left(B^{\mathcal{T}}\left(X_{t_{0}}-v\right)\right)_{\ell} \geqslant\left(B^{\mathcal{T}} v\right)_{\ell} \geqslant \lambda_{\max }(B)^{\mathcal{T}} .
$$

Our choice of $\mathcal{T}$ yields w.h.p. $\left|I_{t+\tau}\right| \geqslant \lambda_{\max }(B)^{\mathcal{T}} \geqslant n / \log n$. Note that, since $v>0$ was chosen arbitrarily, we in fact have that $\tau \leqslant \log _{1+2 q}(n)+o(\log n)$, and the proof is completed. 


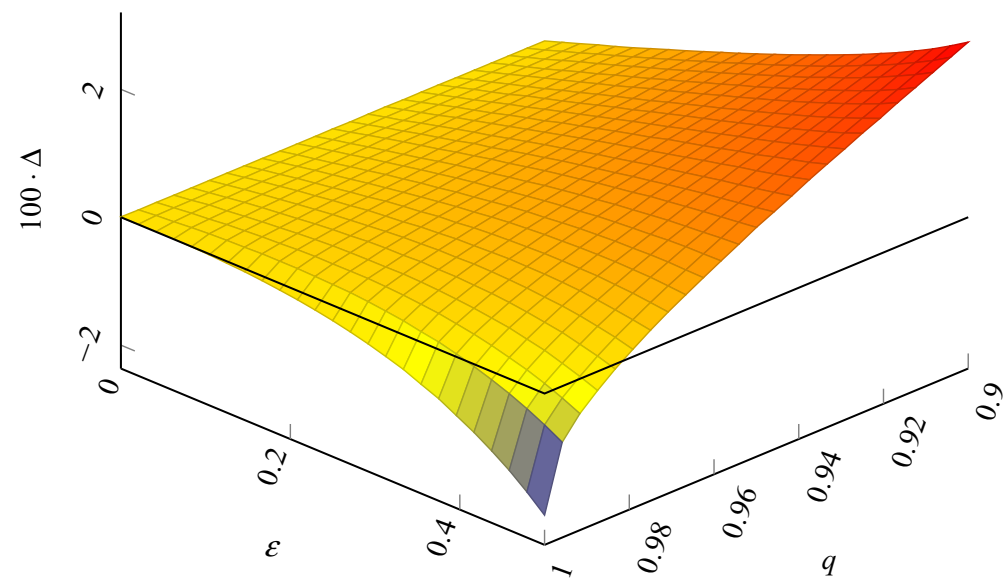

Figure 1. Plotted values of $\Delta$ in $T_{p p}\left(G_{n}(\varepsilon), q\right)-c_{p p} \log n=\Delta \log n+o(\log n)$, for $0.9<q<1$ and $0<\varepsilon<1 / 2$.

\subsection{Proof of Theorem 1.4(b) - edge deletions may slow down push\&pull}

For any $0<\varepsilon<1 / 2, q \in(0,1)$ we consider a sequence of graphs $\left(G_{n}(\varepsilon)\right)_{n \in \mathbb{N}}=\left(\left(V_{n}, E_{n}\right)\right)_{n \in \mathbb{N}}$ that is similar to the one studied in the proof of Theorem 1.4(a). Let $V_{n}=A_{n} \cup B_{n}$ with $A_{n}:=$ $\{1, \ldots,\lfloor n / 2\rfloor\}, B_{n}:=\{\lfloor n / 2\rfloor+1, \ldots, n\}$ and $\operatorname{deg}(v)=n-1$ for all $v \in A_{n}$. Let the induced subgraph of $B_{n}$ be a random graph in which each edge is included independently with probability $p=1-2 \varepsilon$. We know and it is easy to show (see e.g. [15, Section IV]) that w.h.p. this subgraph is almost regular, that is,

$$
d_{B_{n}}(v)=(1+o(1))(1-2 \varepsilon) n / 2 \quad \text { for all } v \in B_{n},
$$

and is an expander, which means that for every $S_{n} \subseteq B_{n}, 1 \leqslant\left|S_{n}\right| \leqslant n / 4$ and $d_{B_{n}}:=(1-2 \varepsilon) n / 2$ we have

$$
e\left(S_{n}, B_{n} \backslash S_{n}\right)=(1+o(1)) \frac{d_{B_{n}}\left|S_{n}\right|\left|B_{n} \backslash S_{n}\right|}{\left|B_{n}\right|}=(1-2 \varepsilon+o(1))\left|S_{n}\right|\left|B_{n} \backslash S_{n}\right| .
$$

First we give a statement that describes the expected number of informed vertices after performing one round of pusherpull.

Lemma 3.16. Let $G_{n}(\varepsilon)=\left(A_{n} \cup B_{n}, E_{n}\right)$ be as above.

(a) Let $\sqrt{\log n} \leqslant\left|I_{t}\right| \leqslant n / \log n$ and set

$$
X_{t}=\left(\left|I_{t}^{(p p),(A)}\right|,\left|I_{t}^{(p p),(B)}\right|\right):=\left(\left|I_{t}^{(p p)} \cap A_{n}\right|,\left|I_{t}^{(p p)} \cap B_{n}\right|\right) .
$$

Then $\mathbb{E}_{t}\left[X_{t+1}\right]=(1+o(1)) M X_{t}$, where

$$
M=\left(\begin{array}{cc}
1+q & q(1+\varepsilon /(2-2 \varepsilon)) \\
q(1+\varepsilon /(2-2 \varepsilon)) & 1+q(1-2 \varepsilon /(2-2 \varepsilon))
\end{array}\right) .
$$

(b) Let $\left|U_{t}^{(p p)}\right| \leqslant n / \log n$. Then

$$
\mathbb{E}_{t}\left[\left|U_{t+1}^{(p p)}\right|\right] \leqslant(1+o(1)) e^{-q(1 / 2+(1 / 2-\varepsilon) /(1-\varepsilon))}(1-q)\left|U_{t}\right| .
$$

Proof. For $J \in\{A, B\}, J_{n} \in\left\{A_{n}, B_{n}\right\}$ set $U_{t}^{(J)}:=U_{t} \cap J_{n}, I_{t}^{(J)}:=I_{t} \cap J_{n}$ and $I_{t+1}^{(p p),(J)}=I_{t+1}^{(p p)} \cap J_{n}$. We first prove (a) by computing the expected number of informed vertices after a single round. Since 
$d(u)=\Theta(n)$ for all $u \in V_{n}$ and $\left|I_{t}\right| \leqslant n / \log n$, the probability of $u \in U_{t}$ being informed by pull is

$$
\mathbb{P}_{t}\left[u \in I_{t+1}^{(\text {pull })} \backslash I_{t}\right]=\frac{q\left|N(u) \cap I_{t}\right|}{|N(u)|}=o(1) .
$$

As the events of $u$ being informed by push and pull are independent, we have

$$
\mathbb{P}_{t}\left[u \in\left(I_{t+1}^{(\text {push })} \cap I_{t+1}^{(\text {pull })}\right) \backslash I_{t}\right]=o(1) \mathbb{P}_{t}\left[u \in I_{t+1}^{(\text {push })} \backslash I_{t}\right] .
$$

Thus

$$
\mathbb{E}_{t}\left[\left|I_{t+1}^{(p p)} \backslash I_{t}\right|\right]=(1+o(1))\left(\mathbb{E}_{t}\left[\left|I_{t+1}^{(\text {push })} \backslash I_{t}\right|\right]+\mathbb{E}_{t}\left[\left|I_{t+1}^{(\text {pull })} \backslash I_{t}\right|\right]\right) .
$$

We look at pull in detail first. Recall that

$$
\operatorname{deg}(v)=n-1 \text { for all } v \in A_{n} \quad \text { and } \quad \operatorname{deg}(v)=(1+o(1))(1-\varepsilon) n \text { for all } v \in B_{n} .
$$

Moreover, using (3.29), we obtain

$$
\begin{aligned}
\mathbb{E}_{t}\left[\left|I_{t+1}^{(\text {pull })} \backslash I_{t}\right|\right] & =\sum_{u \in U_{t}} q \frac{\left|N(u) \cap I_{t}\right|}{|N(u)|} \\
& =\sum_{u \in U_{t}^{(A)}} q \frac{\left|N(u) \cap I_{t}\right|}{|N(u)|}+\sum_{u \in U_{t}^{(B)}} q \frac{\left|N(u) \cap I_{t}\right|}{|N(u)|} \\
& =(q+o(1)) \frac{n}{2}\left(\frac{\left|I_{t}^{(A)}\right|+\left|I_{t}^{(B)}\right|}{n}+\frac{\left|I_{t}^{(A)}\right|+(1-2 \varepsilon)\left|I_{t}^{(B)}\right|}{(1-\varepsilon) n}\right)
\end{aligned}
$$

and thus

$$
\begin{aligned}
& \mathbb{E}_{t}\left[\left|I_{t+1}^{(\text {pull }),(A)} \backslash I_{t}\right|\right]=(q+o(1)) \frac{\left|I_{t}^{(A)}\right|+\left|I_{t}^{(B)}\right|}{2}, \\
& \mathbb{E}_{t}\left[\left|I_{t+1}^{(p u l l),(B)} \backslash I_{t}\right|\right]=(q+o(1)) \frac{\left|I_{t}^{(A)}\right|+(1-2 \varepsilon)\left|I_{t}^{(B)}\right|}{2(1-\varepsilon)} .
\end{aligned}
$$

Next we consider push. By using $\left|I_{t}\right|=o(n)=o\left(\delta_{G_{n}(\varepsilon)}\right)$ and (3.3), we obtain

$$
\begin{aligned}
\mathbb{E}_{t}\left[\left|I_{t+1}^{(\text {push })} \backslash I_{t}\right|\right] & =\sum_{u \in U_{t}} 1-\prod_{i \in N(u) \cap I_{t}}\left(1-\frac{q}{|N(i)|}\right) \\
& =\sum_{u \in U_{t}}(1+o(1)) \sum_{i \in N(u) \cap I_{t}} \frac{q}{|N(i)|} \\
& =(q+o(1)) \sum_{u \in U_{t}}\left(\frac{\left|I_{t}^{(A)}\right|}{n}+\frac{\mathbb{1}\left[u \in U_{t}^{(A)}\right]\left|I_{t}^{(B)}\right|+\mathbb{1}\left[u \in U_{t}^{(B)}\right]\left|N(u) \cap I_{t}^{(B)}\right|}{(1-\varepsilon) n}\right)
\end{aligned}
$$

and thus, with $\left|U_{t}^{(A)}\right|,\left|U_{t}^{(B)}\right|=(1-o(1)) n / 2$ and (3.29),

$$
\begin{aligned}
& \mathbb{E}_{t}\left[\left|I_{t+1}^{(\text {push }),(A)} \backslash I_{t}\right|\right]=(q+o(1))\left(\frac{\left|I_{t}^{(A)}\right|}{2}+\frac{\left|I_{t}^{(B)}\right|}{2}+\frac{\varepsilon\left|I_{t}^{(B)}\right|}{2(1-\varepsilon)}\right), \\
& \mathbb{E}_{t}\left[\left|I_{t+1}^{(\text {push }),(B)} \backslash I_{t}\right|\right]=(q+o(1))\left(\frac{\left|I_{t}^{(A)}\right|}{2}+\frac{\left|I_{t}^{(B)}\right|}{2}-\frac{\varepsilon\left|I_{t}^{(B)}\right|}{2(1-\varepsilon)}\right) .
\end{aligned}
$$

Accumulating the calculated expectations for pull and push yields the claim.

Next we show (b). The assumption implies that $\left|I_{t}\right|=(1-o(1)) n$ and therefore $\left|I_{t}^{(A)}\right|=\left|I_{t}^{(B)}\right|=$ $(1-o(1)) n / 2$. Let $D_{u}$ be the event that an uninformed vertex $u$ does not get informed by the 
push algorithm; let $E_{u}$ be the corresponding event for $p u l l$. Then $D_{u}$ and $E_{u}$ are independent and $D_{u} \cap E_{u}$ is the event that $u$ does not get informed in the current round. Let $u \in U_{t}^{(A)}$, and then

$$
\begin{aligned}
\mathbb{P}_{t}\left[D_{u}\right] & =\prod_{v \in I_{t}^{(A)}}\left(1-\frac{q}{|N(v)|}\right) \prod_{v \in I_{t}^{(B)}}\left(1-\frac{q}{|N(v)|}\right) \\
& =(1-o(1))\left(1-\frac{q}{n}\right)^{\left|I_{t}^{(A)}\right|}\left(1-\frac{q}{(1-\varepsilon) n}\right)^{\left|I_{t}^{(B)}\right|} \\
& =e^{-q(1 / 2+1 /(2(1-\varepsilon)))}+o(1) \\
& \leqslant e^{-q(1 / 2+(1-2 \varepsilon) /(2(1-\varepsilon)))}+o(1)
\end{aligned}
$$

and

$$
\mathbb{P}_{t}\left[E_{u}\right]=1-\frac{q|N(u) \cap| I_{t}||}{|N(u)|}=1-\frac{q\left|I_{t}\right|}{n-1}=1-q+o(1) .
$$

Now consider $u \in U_{t}^{(B)}$; then according to (3.28) we have

$$
\left|N(u) \cap I_{t}^{(B)}\right|=\left|N(u) \cap B_{n}\right|-\left|N(u) \cap U_{t}^{(B)}\right|=(1+o(1))(1-2 \varepsilon) n / 2 .
$$

Therefore

$$
\begin{aligned}
\mathbb{P}_{t}\left[D_{u}\right] & =\prod_{v \in I_{t}^{(A)}}\left(1-\frac{q}{|N(v)|}\right) \prod_{v \in N(u) \cap I_{t}^{(B)}}\left(1-\frac{q}{|N(v)|}\right) \\
& =(1-o(1)) e^{-q / 2}\left(1-\frac{q}{(1-\varepsilon) n}\right)^{\left|N(u) \cap I_{t}^{(B)}\right|} \\
& =e^{-q(1 / 2+(1-2 \varepsilon) /(2(1-\varepsilon)))}+o(1)
\end{aligned}
$$

and

$$
\mathbb{P}_{t}\left[E_{u}\right]=1-\frac{q|N(u) \cap| I_{t}||}{|N(u)|}=1-(1+o(1)) \frac{q\left(\left|I_{t}^{(A)}\right|+\left|N(u) \cap I_{t}^{(B)}\right|\right)}{(1-\varepsilon) n}=1-q+o(1) .
$$

Combining the results for $u \in U_{t}^{(A)}$ and $u \in U_{t}^{(B)}$, we get

$$
\mathbb{E}_{t}\left[\left|U_{t+1}\right|\right]=\sum_{u \in U_{t}} \mathbb{P}_{t}\left[D_{u}\right] \mathbb{P}_{t}\left[E_{u}\right] \leqslant(1+o(1)) e^{-q(1 / 2+(1 / 2-\varepsilon) /(1-\varepsilon))}(1-q)\left|U_{t}\right| .
$$

Remark 3.1. Let $\lambda_{\max }$ be the greatest eigenvalue of $M$ as defined in Lemma 3.16(a). Then

$$
\lambda_{\max }=1+2 q+\left(2 q\left(\sqrt{\left(\varepsilon^{2} / 2-\varepsilon+1\right)}-1\right)+q \varepsilon\right) /(2-2 \varepsilon)>1+2 q .
$$

Next comes a lemma that bounds the runtime of pusherpull on $G_{n}(\varepsilon)$. In particular, (a) and (c) of Lemma 3.17 provide a lower bound on the runtime, and (a), (b) and (d) of Lemma 3.17 together with Lemma 3.15(a) provide an upper bound.

Lemma 3.17. Let $I_{t}=I_{t}^{(p p)}, \varepsilon>0$ and $\lambda=\lambda_{\max }(M)$ be the greatest eigenvalue of $M$ as given in Lemma 3.16(a). Consider $G_{n}(\varepsilon)$.

(a) Let $\sqrt{\log n} \leqslant\left|I_{t}\right| \leqslant n / \log n$. Then there are $\tau_{1}, \tau_{2}=\log _{\lambda}\left(n /\left|I_{t}\right|\right)+o(\log n)$ such that $\left|I_{t+\tau_{1}}\right|<n / \log n<\left|I_{t+\tau_{1}}\right|$. 
(b) Let $n / \log n \leqslant\left|I_{t}\right| \leqslant n-n / \log n$. Then there is $\tau=o(\log n)$ such that $\left|I_{t+\tau}\right|>n-n / \log n$.

(c) Let $\left|I_{t}\right| \leqslant n / \log n$. Then there is

$$
\tau \geqslant \log n / \log \left((1-q)^{-1} \exp (q(1 / 2+(1 / 2-\varepsilon) /(1-\varepsilon)))\right)-o(\log n)
$$

such that $\left|I_{t+\tau}\right|<n$.

(d) Let $\left|I_{t}\right| \geqslant n-n / \log n$ and $q \in(0,1)$. Then there is

$$
\tau \leqslant \log n / \log \left((1-q)^{-1} \exp (q(1 / 2+(1 / 2-\varepsilon) /(1-\varepsilon)))\right)+o(\log n)
$$

such that $\left|I_{t+\tau}\right|=n$.

Proof. We do not give a proof for (b) as it follows immediately from Lemma 3.15(a). For $J \in\{A, B\}$ set $U_{t}^{(J)}:=U_{t} \cap J_{n}, I_{t}^{(J)}:=I_{t} \cap J_{n}$. We prove (a) first. Let $t_{0}>0$ be the first round such that $\left|I_{t_{0}}\right| \geqslant \log \log n$ and set $X_{t}$ and $M$ as in Lemma 3.16(a); note that Lemma 3.15(a) also gives that $\left(X_{t_{0}}\right)_{i} \geqslant \log \log n / 2$ for $i \in\{1,2\}$. Then, for all $t \geqslant t_{0}$ such that $\left|I_{t}\right| \leqslant n / \log n$, we obtain from Lemma 3.16(a) that $\mathbb{E}_{t}\left[X_{t+1}\right]=(1+o(1)) M X_{t}$ and, in particular, $\mathbb{E}_{t}\left[\left(X_{t+1}\right)_{i}\right]=\Theta\left(\left|I_{t}\right|\right)$ for $i \in\{1,2\}$. As every component of $X_{t}$ is self-bounding, Lemma 2.1 applies and we get for $i \in\{1,2\}$

$$
\mathbb{P}_{t}\left[\left|\left(X_{t+1}\right)_{i}-\mathbb{E}_{t}\left[\left(X_{t+1}\right)_{i}\right]\right| \geqslant \mathbb{E}_{t}\left[\left(X_{t+1}\right)_{i}\right]^{2 / 3}\right]=O\left(\left|I_{t}\right|^{-1 / 3}\right)
$$

and by the union bound, provided that $\left|I_{t}\right| \leqslant n / \log n$,

$$
\mathbb{P}_{t}\left[\bigcap_{i \in\{1,2\}}\left(\left|\left(X_{t+1}\right)_{i}-\mathbb{E}_{t}\left[\left(X_{t+1}\right)_{i}\right]\right| \leqslant \mathbb{E}_{t}\left[\left(X_{t+1}\right)_{i}\right]^{2 / 3}\right)\right]=1-O\left(\left|I_{t}\right|^{-1 / 3}\right) .
$$

Using (3.30) we want to find a bound on $\left|I_{t+1}\right|$. As long as $\left|I_{t}\right| \leqslant n / \log n$, we get

$$
\left(\left(1-O\left(\left|I_{t_{0}}\right|^{-1 / 3}\right)\right) M\right)^{t+1-t_{0}} X_{t_{0}} \leqslant X_{t+1} \leqslant\left(\left(1+O\left(\left|I_{t_{0}}\right|^{-1 / 3}\right)\right) M\right)^{t+1-t_{0}} X_{t_{0}} .
$$

As seen in Remark 3.1, $M$ has maximal eigenvalue $\lambda_{\max }>1$, and as $M$ is a positive matrix there is a positive eigenvector $v$ to $\lambda_{\max }$; see [32]. This gives constants $c_{1}, c_{2}>0$ such that $c_{1} v \log \log n \leqslant$ $X_{t_{0}} \leqslant c_{2} v \log \log n$, and for $t$ large enough

$$
\frac{c_{1}}{c_{2}}\left(\left(1-O\left(\left|I_{t_{0}}\right|^{-1 / 3}\right)\right) \lambda_{\max }\right)^{t+1-t_{0}} X_{t_{0}} \leqslant X_{t+1} \leqslant \frac{c_{2}}{c_{1}}\left(\left(1+O\left(\left|I_{t_{0}}\right|^{-1 / 3}\right)\right) \lambda_{\max }\right)^{t+1-t_{0}} X_{t_{0}},
$$

and therefore

$$
\left|I_{t+1}\right| \leqslant \frac{c_{1}}{c_{2}}\left((1+o(1)) \lambda_{\max }\right)^{t-t_{0}}\left|I_{t_{0}}\right|
$$

as long as the right-hand side is bounded by $n / \log n$. For all these $t$ we also get

$$
\left|I_{t+1}\right| \geqslant \frac{c_{2}}{c_{1}}\left((1-o(1)) \lambda_{\max }\right)^{t-t_{0}}\left|I_{t_{0}}\right|
$$

Proceeding as in Lemmas 2.5 and 2.6 and their proofs, where we replace the events

$$
\left|I_{t}\right| \geqslant \mathbb{E}_{t-1}\left[\left|I_{t}\right|\right]-\mathbb{E}_{t-1}\left[\left|I_{t}\right|\right]^{2 / 3} \text { or }\left|I_{t}\right| \geqslant n / g(n)
$$

and

$$
|| I_{t}\left|-\mathbb{E}_{t-1}\left[\left|I_{t}\right|\right]\right| \leqslant \mathbb{E}_{t-1}\left[\left|I_{t}\right|\right]^{2 / 3}
$$

with

$$
\bigcap_{i \in\{1,2\}}\left(\left(X_{t+1}\right)_{i} \geqslant\left(1-\mathbb{E}_{t}\left[\left(X_{t+1}\right)_{i}\right]^{-1 / 3}\right) \mathbb{E}_{t}\left[\left(X_{t+1}\right)_{i}\right]\right) \quad \text { or } \quad\left|I_{t}\right| \geqslant n / \log n
$$

and

$$
\bigcap_{i \in\{1,2\}}\left(\left|\left(X_{t+1}\right)_{i}-\mathbb{E}_{t}\left[\left(X_{t+1}\right)_{i}\right]\right| \leqslant \mathbb{E}_{t}\left[\left(X_{t+1}\right)_{i}\right]^{2 / 3}\right)
$$


we obtain the statement. Next we show (c). The assumption guarantees that less than $n / \log n$ vertices are informed. Thus $\left|U_{t}^{(B)}\right| \geqslant n / 2-\left|I_{t}\right| \geqslant(1 / 2-1 / \log n) n$. We consider a modified dissemination process, where in each round, each uninformed vertex always chooses an informed neighbour (but does not necessarily get informed as the message transmission may fail), and additionally each vertex chooses a neighbour independently and uniformly at random and after this round the chosen vertex is informed with probability $q$; in other words, we assume that uninformed vertices can inform other vertices. In this modified process the probability of an uninformed vertex $u \in U_{t}^{(B)}$ staying uninformed after performing one round is given by the product of the probabilities of not being informed by pull or via push by a vertex in $A_{n}$ or $B_{n}$. Using (3.29) and $(1-1 / n)^{n}=e^{-1+o(1)}$, we get $g(n)=o(1)$ such that

$$
\begin{aligned}
\mathbb{P}_{t}\left[u \in U_{t+1}^{(B)}\right] & =(1-q)\left(1-\frac{q}{n}\right)^{n / 2}\left(1-\frac{q}{(1-\varepsilon) n}\right)^{\left|N(u) \cap B_{n}\right|} \\
& =(1-q) \exp \left(-q\left(\frac{1}{2}+\frac{1 / 2-\varepsilon}{1-\varepsilon}\right)+g(n)\right) .
\end{aligned}
$$

As we have seen in the proof of Lemma 3.16(b), the probability of being informed by pushepull is greater for a vertex in $A_{n}$ than for a vertex in $B_{n}$. Therefore it is sensible to expect that some vertices in $B_{n}$ will be the last to be informed. Consequently let $E_{\mathcal{u}}$ denote the event that a currently uninformed vertex $u \in U_{t}^{(B)}$ does not get informed in this modified version within the next

$$
\tau:=\frac{1}{\log \left((1-q)^{-1} \exp (q(1 / 2+(1 / 2-\varepsilon) /(1-\varepsilon)-g(n)))\right)} \log (n)-h(n)
$$

rounds, where $h=o(\log n)$ and $h=\omega(1)$. Therefore we have

$$
\mathbb{P}_{t}\left[E_{u}\right]=\left((1-q) \exp \left(-q\left(\frac{1}{2}+\frac{1 / 2-\varepsilon}{1-\varepsilon}\right)+g(n)\right)\right)^{\tau}=\frac{1}{n} e^{\omega(1)} .
$$

In this modified model the events $\left\{E_{u} \mid u \in U_{t}^{(B)}\right\}$ satisfy that there is $p=\omega\left(n^{-1}\right)$ such that

$$
\mathbb{P}_{t}\left[E_{u} \mid\left\{\overline{E_{v}}: v \in U\right\}\right] \geqslant p \quad \text { for all } u \in B_{n} \text { and } U \subseteq V \backslash\{u\}
$$

This follows immediately by the above calculations. Thus, as $\left|U_{t}^{(B)}\right|=\Theta(n)$,

$$
\mathbb{P}_{t}\left[\bigwedge_{u \in U_{t}^{(B)}} \overline{E_{u}}\right] \leqslant \prod_{u \in U_{t}^{(B)}}(1-p) \leqslant \exp \left(-\sum_{u \in U_{t}^{(B)}} p\right)=o(1)
$$

Finally we show (d). By Lemma 3.16(b), we obtain that for any $\tau \in \mathbb{N}$,

$$
\mathbb{E}_{t}\left[\left|U_{t+\tau}\right|\right] \leqslant\left((1+o(1)) e^{-q(1 / 2+(1 / 2-\varepsilon) /(1-\varepsilon))}(1-q)\right)^{\tau}\left|U_{t}\right| .
$$

Then, for some

$$
\tau:=\frac{\log (n)}{\log \left((1-q)^{-1} \exp (q(1 / 2+(1 / 2-\varepsilon) /(1-\varepsilon)))\right)}+o(\log n),
$$

we obtain that, say, $\mathbb{E}_{t}\left[\left|U_{t+\tau}\right|\right] \leqslant\left|U_{t}\right| / n \leqslant 1 / \log n$. Thus $\mathbb{P}_{t}\left[\left|U_{t+\tau}\right| \geqslant 1\right] \leqslant o(1)$ by Markov's inequality.

Lemma 3.17 together with Lemma 2.11 gives that

$$
T_{p p}\left(G_{n}(\varepsilon), q\right)=\log _{\lambda} n+\frac{1}{q(1-1.5 \varepsilon) /(1-\varepsilon)-\log (1-q)} \log n+o(\log n),
$$


where

$$
\lambda=1+2 q+\left(2 q\left(\sqrt{\left(\varepsilon^{2} / 2-\varepsilon+1\right)}-1\right)+q \varepsilon\right) /(2-2 \varepsilon)>1+2 q .
$$

To see whether pusherpull actually slowed down (in terms of order $\log n$ ) one has to compare the runtime on this sequence of graphs to $c_{p p} \log n$, the runtime on expander sequences. In Figure 1 we can see that it slows down for nearly all values of $\varepsilon$ and $q$ in question; however, there are admissible values of $\varepsilon$ and $q$ such that the process even speeds up.

\section{References}

[1] Acan, H., Collevecchio, A., Mehrabian, A. and Wormald, N. (2017) On the push\&pull protocol for rumour spreading. In Extended Abstracts Summer 2015 (J. Díaz et al., eds), pp. 3-10. Springer.

[2] Angel, O., Mehrabian, A. and Peres, Y. (2017) The string of diamonds is tight for rumor spreading. In Approximation, Randomization, and Combinatorial Optimization: Algorithms and Techniques (APPROX/RANDOM 2017), pp. 26:126:9. Schloss Dagstuhl-Leibniz-Zentrum für Informatik.

[3] Boucheron, S., Lugosi, G. and Bousquet, O. (2004) Concentration inequalities. In Advanced Lectures on Machine Learning, Vol. 3176 of Lecture Notes in Computer Science, pp. 208-240. Springer.

[4] Boyd, S., Ghosh, A., Prabhakar, B. and Shah, D. (2006) Randomized gossip algorithms. IEEE/ACM Trans. Inform. Theory 52 2508-2530.

[5] Censor-Hillel, K., Haeupler, B., Kelner, J. and Maymounkov, P. (2012) Global computation in a poorly connected world: fast rumor spreading with no dependence on conductance. In Proceedings of the Forty-Fourth Annual ACM Symposium on Theory of Computing (STOC '12), pp. 961-970. ACM.

[6] Chierichetti, F., Giakkoupis, G., Lattanzi, S. and Panconesi, A. (2018) Rumor spreading and conductance. J. Assoc. Comput. Mach. 6517.

[7] Daum, S., Kuhn, F. and Maus, Y. (2016) Rumor spreading with bounded in-degree. In Structural Information and Communication Complexity: 23rd International Colloquium (SIROCCO 2016), pp. 323-339.

[8] Dellamonica, D., Kohayakawa, Y., Marciniszyn, M. and Steger, A. (2008) On the resilience of long cycles in random graphs. Electron. J. Combin. 15 \#R321.

[9] Demers, A., Greene, D., Houser, C., Irish, W., Larson, J., Shenker, S., Sturgis, H., Swinehart, D. and Terry, D. (1988) Epidemic algorithms for replicated database maintenance. ACM SIGOPS Operating Systems Review 22 8-32.

[10] Doerr, B., Fouz, M. and Friedrich, T. (2011) Social networks spread rumors in sublogarithmic time. In Proceedings of the Forty-Third Annual ACM Symposium on Theory of Computing (STOC '11), pp. 21-30. ACM.

[11] Doerr, B. and Kostrygin, A. (2017) Randomized rumor spreading revisited. In 44th International Colloquium on Automata, Languages, and Programming (ICALP 2017), pp. 138:1-138:14.

[12] Doerr, B. and Künnemann, M. (2014) Tight analysis of randomized rumor spreading in complete graphs. In Proceedings of the Meeting on Analytic Algorithmics and Combinatorics (ANALCO 2014), pp. 82-91. SIAM.

[13] Elsässer, R. and Sauerwald, T. (2009) On the runtime and robustness of randomized broadcasting. Theoret. Comput. Sci. $4103414-3427$.

[14] Feige, U., Peleg, D., Raghavan, P. and Upfal, E. (1990) Randomized broadcast in networks. Random Struct. Algorithms $1447-460$.

[15] Fountoulakis, N., Huber, A. and Panagiotou, K. (2010) Reliable broadcasting in random networks and the effect of density. In 2010 Proceedings IEEE INFOCOM, pp. 1-9. IEEE.

[16] Fountoulakis, N. and Panagiotou, K. (2010) Rumor spreading on random regular graphs and expanders. In Approximation, Randomization, and Combinatorial Optimization: Algorithms and Techniques (RANDOM 2010), Vol. 6302 of Lecture Notes in Computer Science, pp. 560-573. Springer.

[17] Fountoulakis, N., Panagiotou, K. and Sauerwald, T. (2012) Ultra-fast rumor spreading in social networks. In Proceedings of the Twenty-Third Annual ACM-SIAM Symposium on Discrete Algorithms (SODA '12), pp. 1642-1660. SIAM.

[18] Friedrich, T., Sauerwald, T. and Stauffer, A. (2013) Diameter and broadcast time of random geometric graphs in arbitrary dimensions. Algorithmica 67 65-88.

[19] Frieze, A. M. and Grimmett, G. R. (1985) The shortest-path problem for graphs with random arc-lengths. Discrete Appl. Math. 10 57-77.

[20] Giakkoupis, G. (2011) Tight bounds for rumor spreading in graphs of a given conductance. In 28th International Symposium on Theoretical Aspects of Computer Science (STACS 2011), pp. 57-68. Schloss Dagstuhl-Leibniz-Zentrum für Informatik

[21] Giakkoupis, G. (2014) Tight bounds for rumor spreading with vertex expansion. In Proceedings of the Twenty-Fifth Annual ACM-SIAM Symposium on Discrete Algorithm (SODA 2014), pp. 801-815. 
[22] Greenberg, S. and Mohri, M. (2014) Tight lower bound on the probability of a binomial exceeding its expectation. Statist. Probab. Lett. 86 91-98.

[23] Haeupler, B. (2015) Simple, fast and deterministic gossip and rumor spreading. J. Assoc. Comput. Mach. 6247.

[24] Hoffman, A. J. and Wielandt, H. W. (1953) The variation of the spectrum of a normal matrix. Duke Math. J. 20 37-39.

[25] Hoory, S., Linial, N. and Wigderson, A. (2006) Expander graphs and their applications. Bull. Amer. Math. Soc. 43 439-561.

[26] Janson, S. (2018) Tail bounds for sums of geometric and exponential variables. Statistics \& Probability Letters, 135 1-6.

[27] Karp, R. M., Schindelhauer, C., Shenker, S. and Vöcking, B. (2000) Randomized rumor spreading. In 41st Annual Symposium on Foundations of Computer Science (FOCS 2000), pp. 565-574.

[28] Panagiotou, K., Pérez-Giménez, X., Sauerwald, T. and Sun, H. (2015) Randomized rumour spreading: the effect of the network topology. Combin. Probab. Comput. 24 457-479.

[29] Panagiotou, K. and Speidel, L. (2017) Asynchronous rumor spreading on random graphs. Algorithmica 78 968-989.

[30] Rödl, V. and Schacht, M. (2010) Regularity lemmas for graphs. In Fete of Combinatorics and Computer Science, Vol. 20 of Bolyai Society Mathematical Studies, pp. 287-325. Springer.

[31] Sudakov, B. and Vu, V. H. (2008) Local resilience of graphs. Random Struct. Algorithms 33 409-433.

[32] Wielandt, H. (1950) Unzerlegbare, nicht negative Matrizen. Math. Z. 52 642-648.

Cite this article: Daknama R, Panagiotou K and Reisser S (2021). Robustness of randomized rumour spreading. Combinatorics, Probability and Computing 30, 37-78. https://doi.org/10.1017/S0963548320000310 